\title{
TSBP: Tangent Space Belief Propagation for Manifold Learning
}

Thomas Cohn, Odest Chadwicke Jenkins, Karthik Desingh, Zhen Zeng

\section{IROS 2020}

Laboratory for

Perception RObotics and Grounded REasoning SystemS 


\section{Robot Sensing Today}

Robots now have access to increasingly detailed sensor data

- High resolution images 


\section{Robot Sensing Today}

Robots now have access to increasingly detailed sensor data

- High resolution images

- Dense point clouds

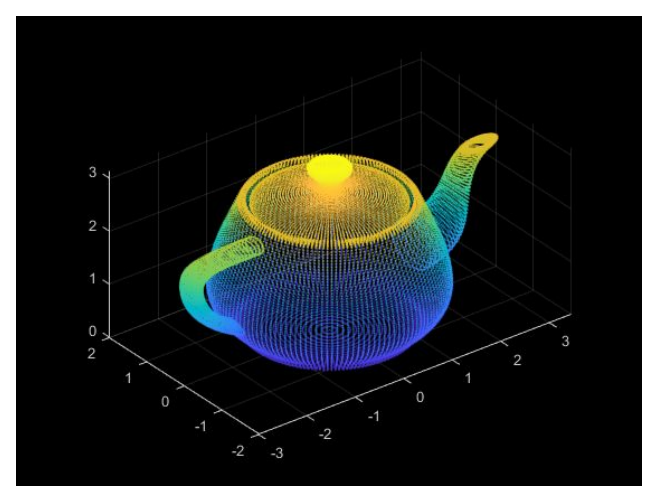

IMAGE: MathWorks 


\section{Robot Sensing Today}

Robots now have access to increasingly detailed sensor data

- High resolution images

- Dense point clouds

- Motion capture data

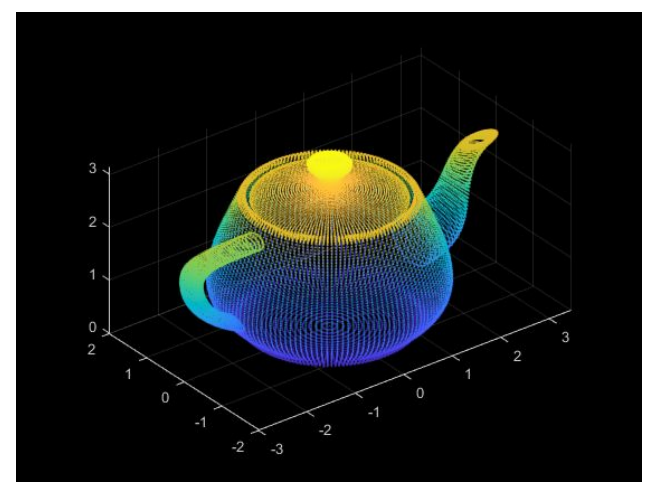

IMAGE: MathWorks

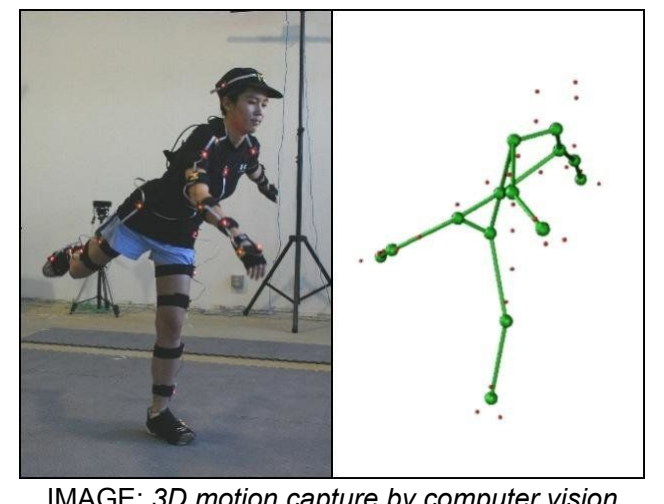

IMAGE: 3D motion capture by computer vision and virtual rendering (D Jáuregui) 


\section{Robot Sensing Today}

Robots now have access to increasingly detailed sensor data

- High resolution images

- Dense point clouds

- Motion capture data

- Tactile information

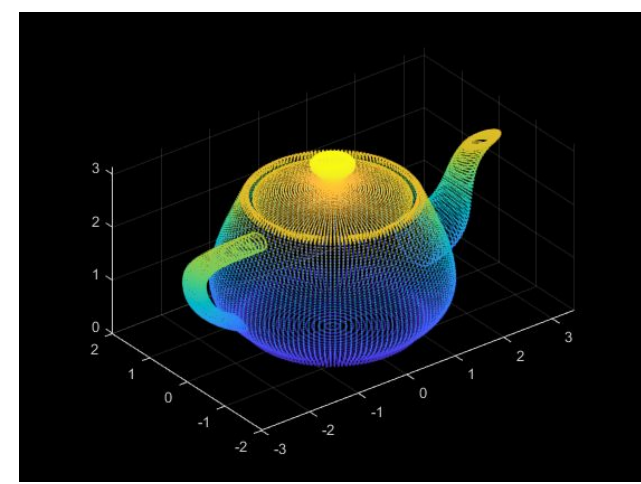

IMAGE: MathWorks

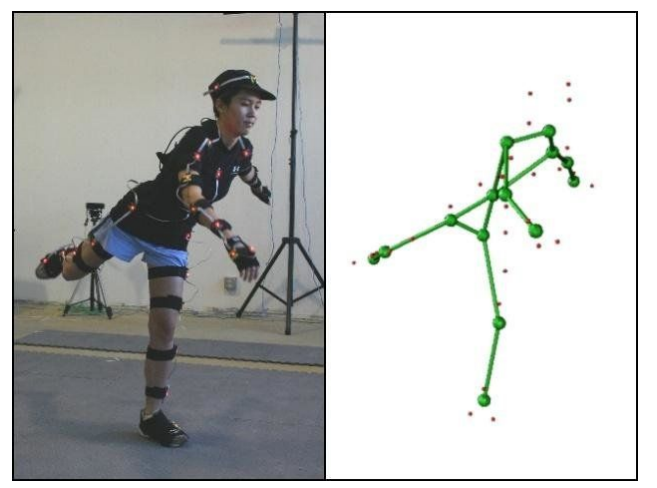

IMAGE: 3D motion capture by computer vision and virtual rendering (D Jáuregui)

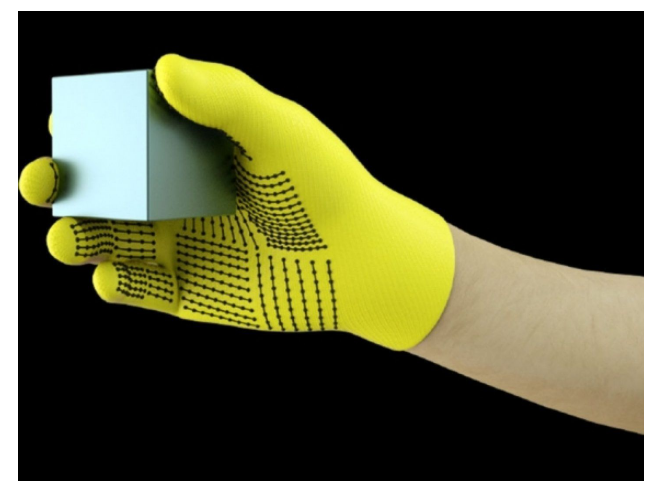

IMAGE: Learning the signatures of the human grasp using a scalable tactile glove (S Sundaram et al) 


\section{The Challenges of High Dimensional Data}

- Some problems and algorithms quickly become intractable

- Partially Observable Markov Decision Processes (POMDPs)

- Particle Filters

$\circ$ Reinforcement Learning 


\section{The Challenges of High Dimensional Data}

- Some problems and algorithms quickly become intractable

- Partially Observable Markov Decision Processes (POMDPs)

- Particle Filters

- Reinforcement Learning

- Interfacing with humans

- Data visualization

- Robot teleoperation 


\section{The Challenges of High Dimensional Data}

- Some problems and algorithms quickly become intractable

- Partially Observable Markov Decision Processes (POMDPs)

- Particle Filters

- Reinforcement Learning

- Interfacing with humans

- Data visualization

- Robot teleoperation

- Data sparsity grows exponentially with dimension

- Hughes Phenomenon: more features can harm accuracy

- Handling bias-variance tradeoff

- Outlier detection 


\section{Dimensionality Reduction to the Rescue}

Reduce the dimension of the data while preserving latent information 


\section{Dimensionality Reduction to the Rescue}

Reduce the dimension of the data while preserving latent information

1. A Spatio-temporal Extension to Isomap Nonlinear Dimension Reduction (OC Jenkins et al)

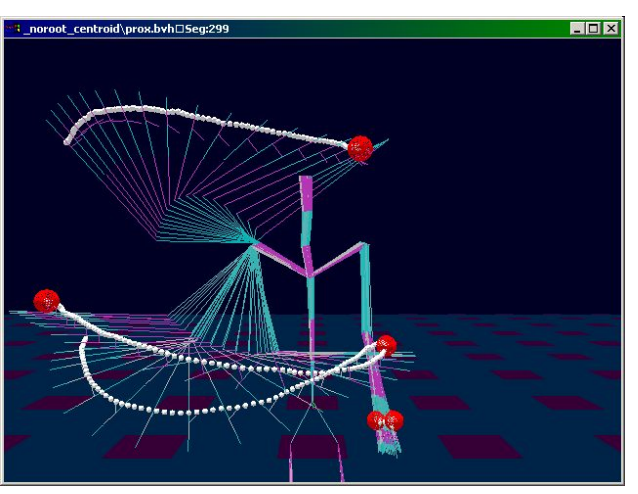

1. Human Motion Capture Data 


\section{Dimensionality Reduction to the Rescue}

Reduce the dimension of the data while preserving latent information

1. A Spatio-temporal Extension to Isomap Nonlinear Dimension Reduction (OC Jenkins et al)

2. Extracting Postural Synergies for Robotic Grasping (J Romero et al)

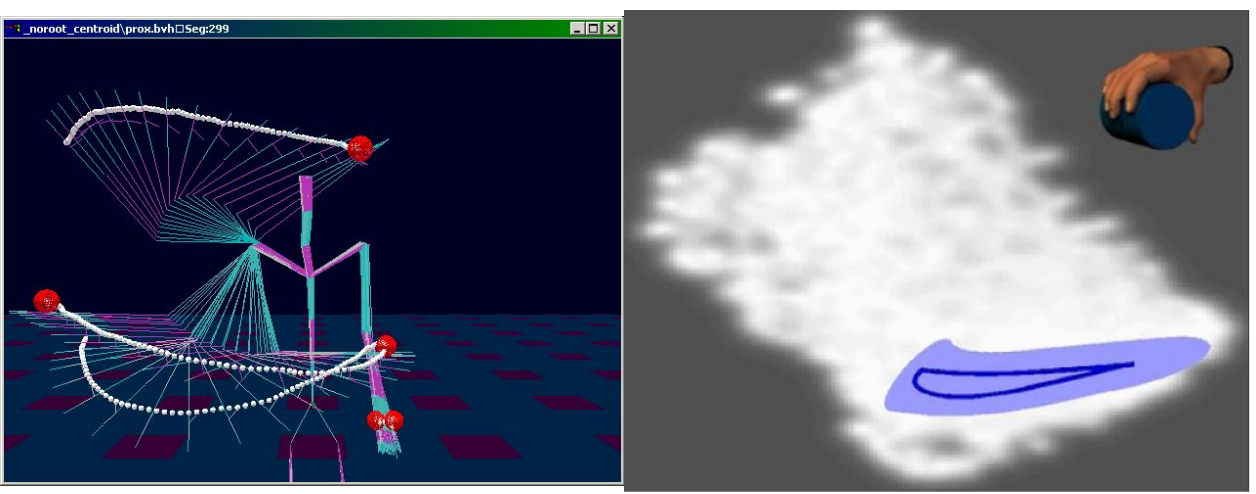

1. Human Motion Capture Data 


\section{Dimensionality Reduction to the Rescue}

Reduce the dimension of the data while preserving latent information

1. A Spatio-temporal Extension to Isomap Nonlinear Dimension Reduction (OC Jenkins et al)

2. Extracting Postural Synergies for Robotic Grasping (J Romero et al)

3. Detecting the Functional Similarities Between Tools Using a Hierarchical Representation of Outcomes (J Sinapov et al)

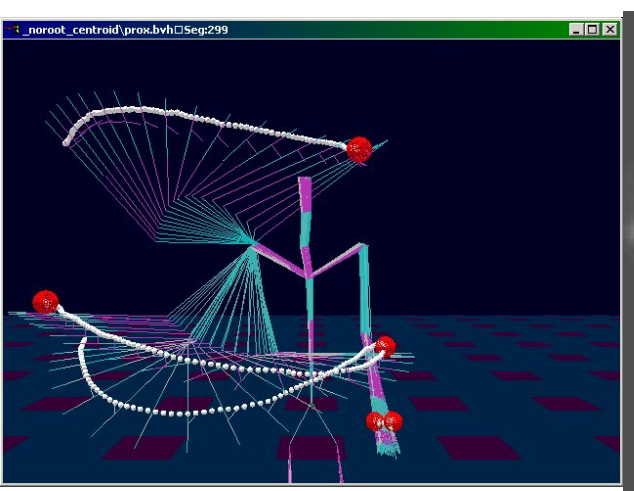

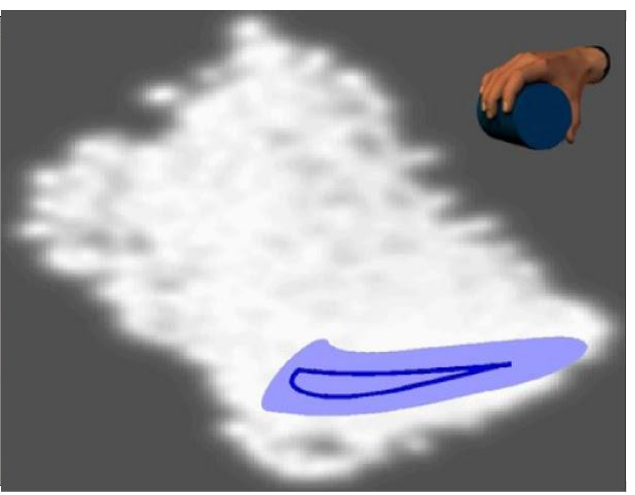

2. Grasp Trajectory
1. Human Motion Capture Data

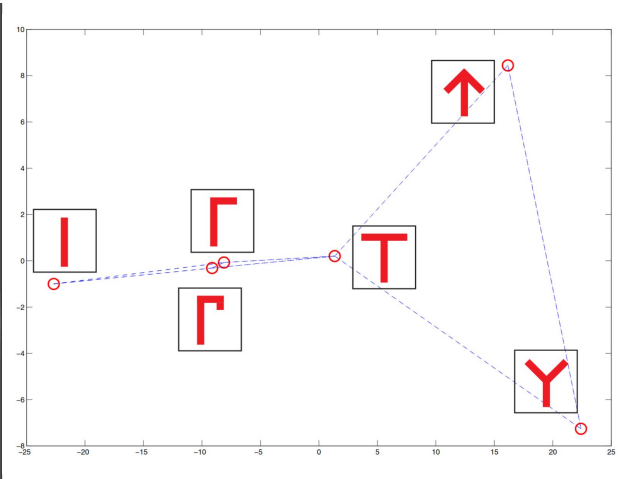

3. Tool Shape Similarities 


\section{Dimensionality Reduction}

- Transform data to a lower-dimensional space

- Preserve data similarities/dissimilarities 


\section{Dimensionality Reduction}

- Transform data to a lower-dimensional space

- Preserve data similarities/dissimilarities
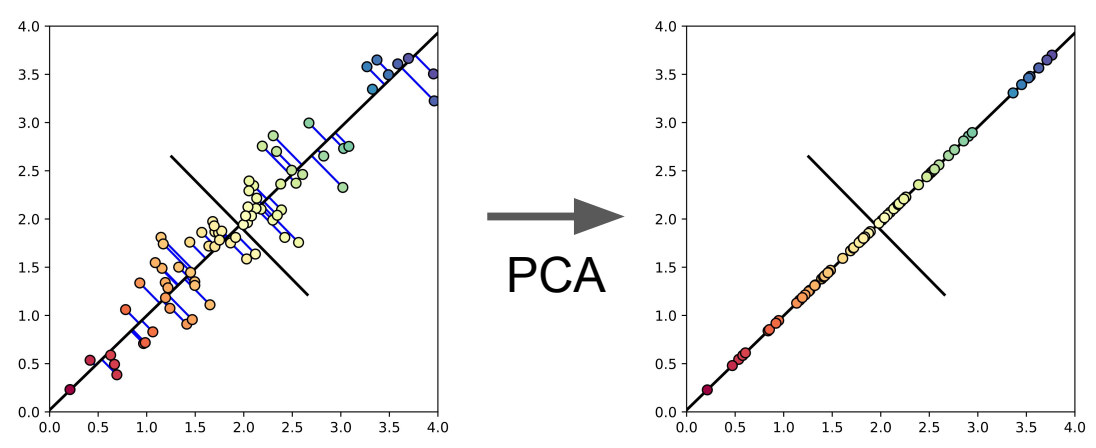


\section{Dimensionality Reduction}

- Transform data to a lower-dimensional space

- Preserve data similarities/dissimilarities
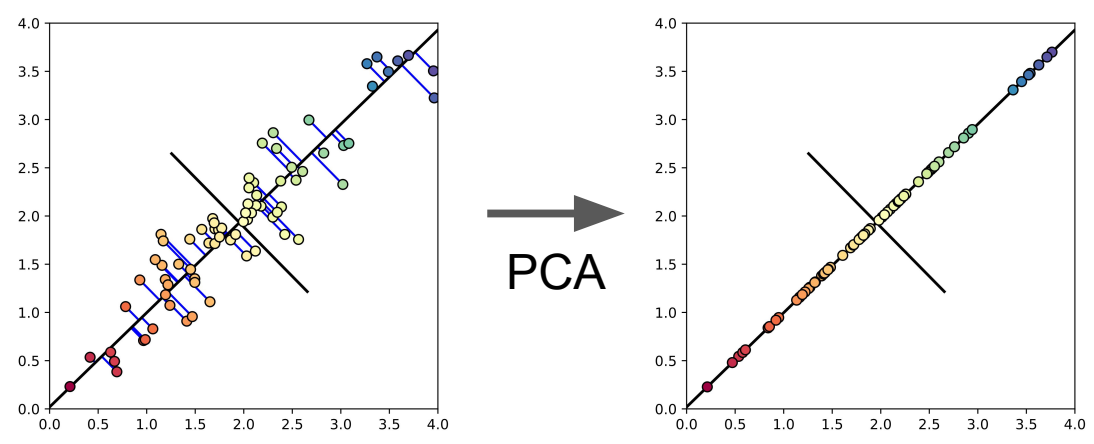

Deep Autoencoder

$$
\text { Input Encoding DBN }
$$




\section{Dimensionality Reduction}

- Transform data to a lower-dimensional space

- Preserve data similarities/dissimilarities
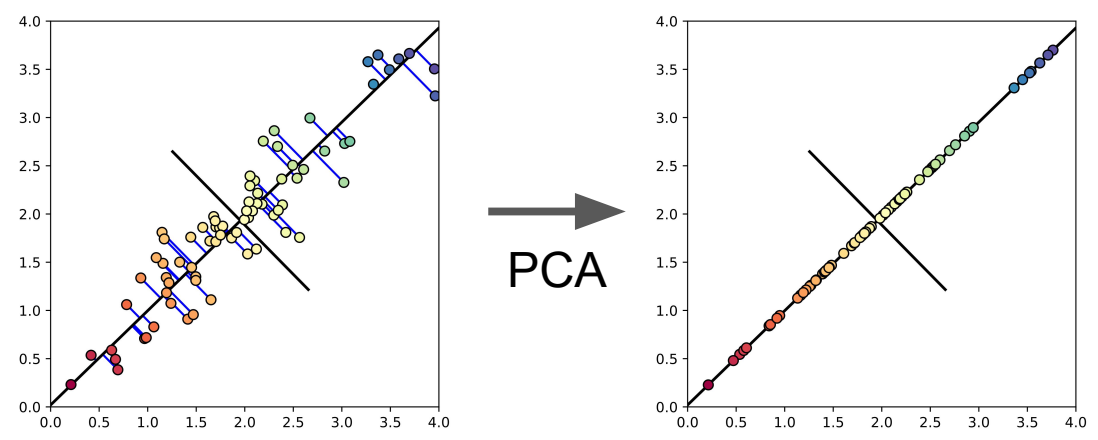

Deep Autoencoder

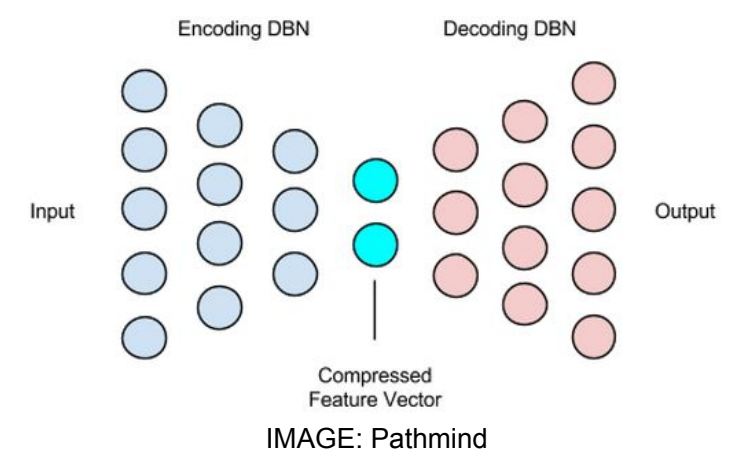

- Model data as lying along a manifold

- Learn a low-dimensional embedding 


\section{Dimensionality Reduction}

- Transform data to a lower-dimensional space

- Preserve data similarities/dissimilarities
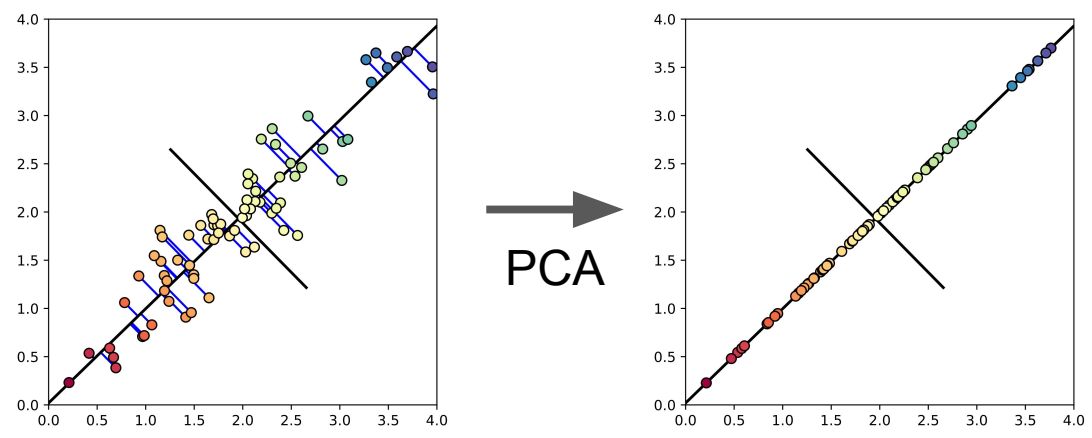

Deep Autoencoder

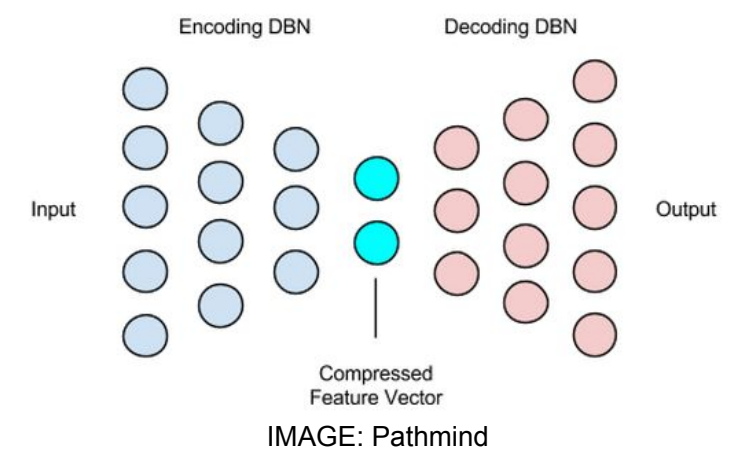

- Model data as lying along a manifold

- Learn a low-dimensional embedding

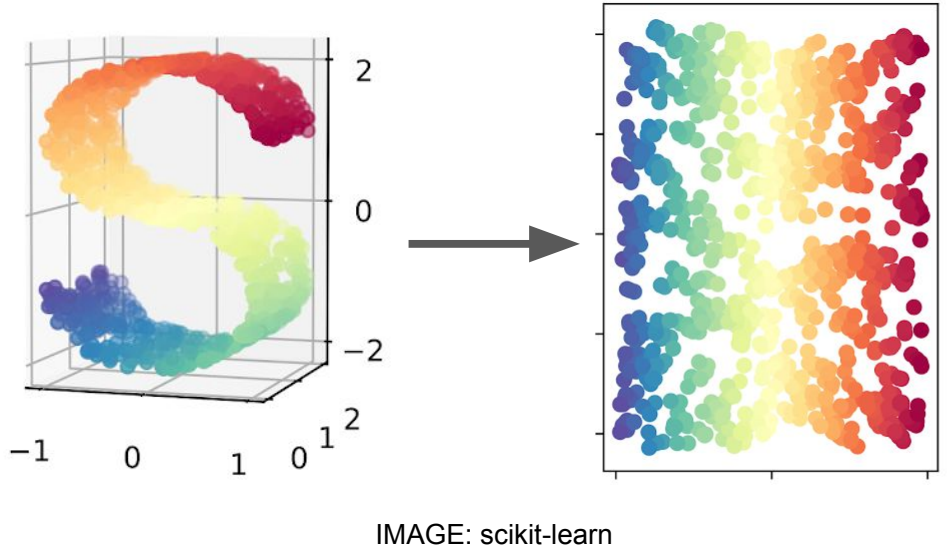




\section{Nearest-Neighbors}

- Common tool to discover local structure

- Used as a starting point by many manifold learning algorithms
- Isometric Mapping (ISOMAP)
- Locally Linear Embedding (LLE)
- Laplacian Eigenmaps (LE)
- Local Tangent Space Alignment (LTSA) 


\section{Nearest-neighbors}

- Can be "short-circuited" by false edges, caused by

- Noisy or sparse data

- Distinct regions of the manifold passing close to one another

- Makes points falsely appear highly similar 


\section{Nearest-neighbors}

- Can be "short-circuited" by false edges, caused by

- Noisy or sparse data

- Distinct regions of the manifold passing close to one another

- Makes points falsely appear highly similar

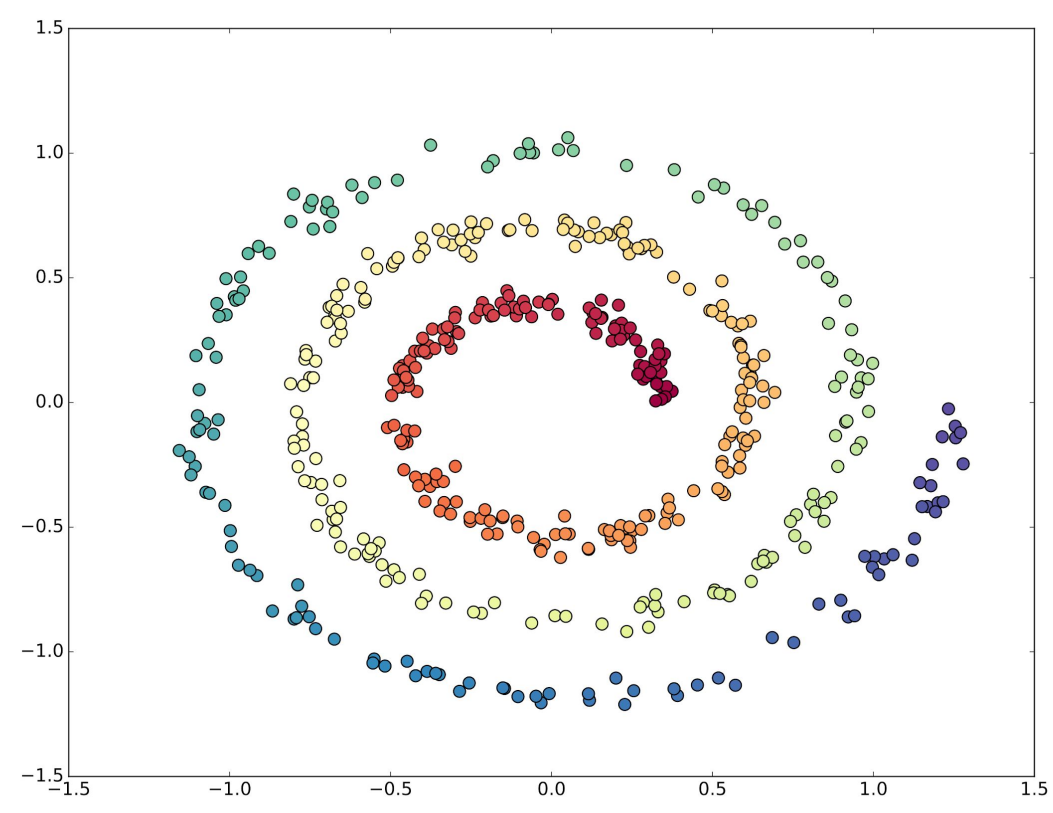




\section{Nearest-neighbors}

- Can be "short-circuited" by false edges, caused by

- Noisy or sparse data

- Distinct regions of the manifold passing close to one another

- Makes points falsely appear highly similar

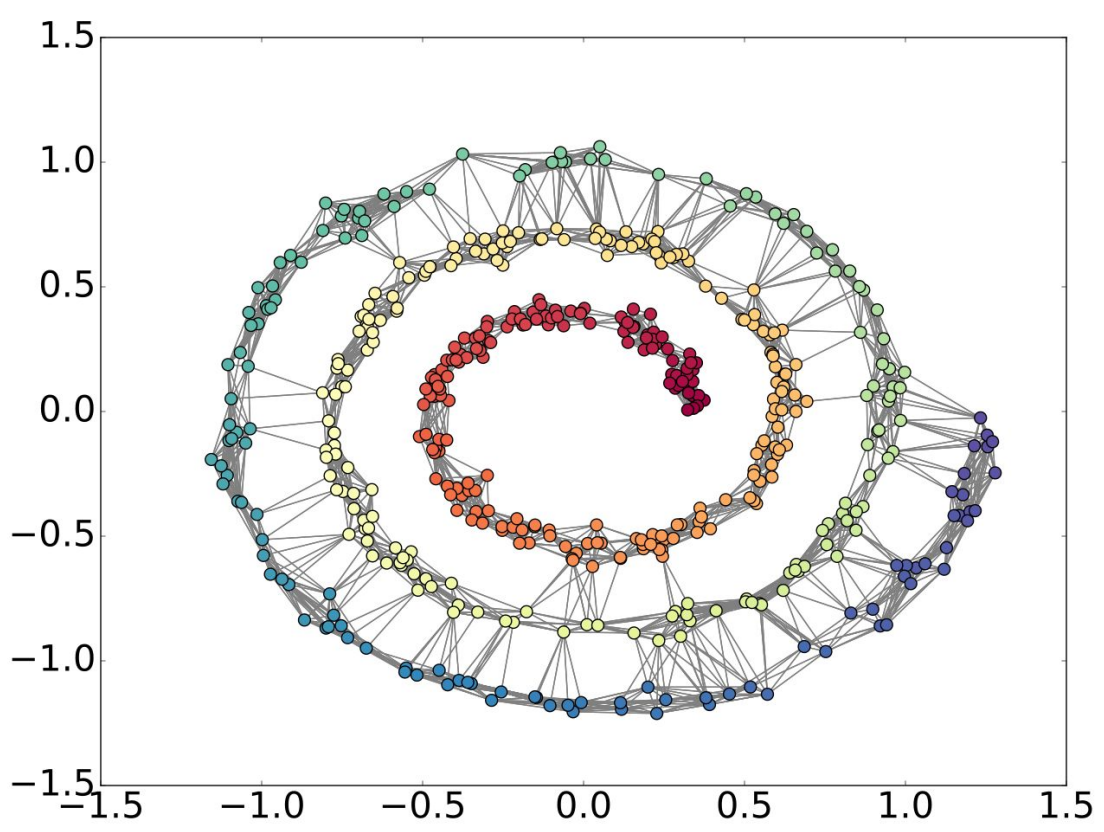




\section{Nearest-neighbors}

- Can be "short-circuited" by false edges, caused by

- Noisy or sparse data

- Distinct regions of the manifold passing close to one another

- Makes points falsely appear highly similar

- Want to remove false edges

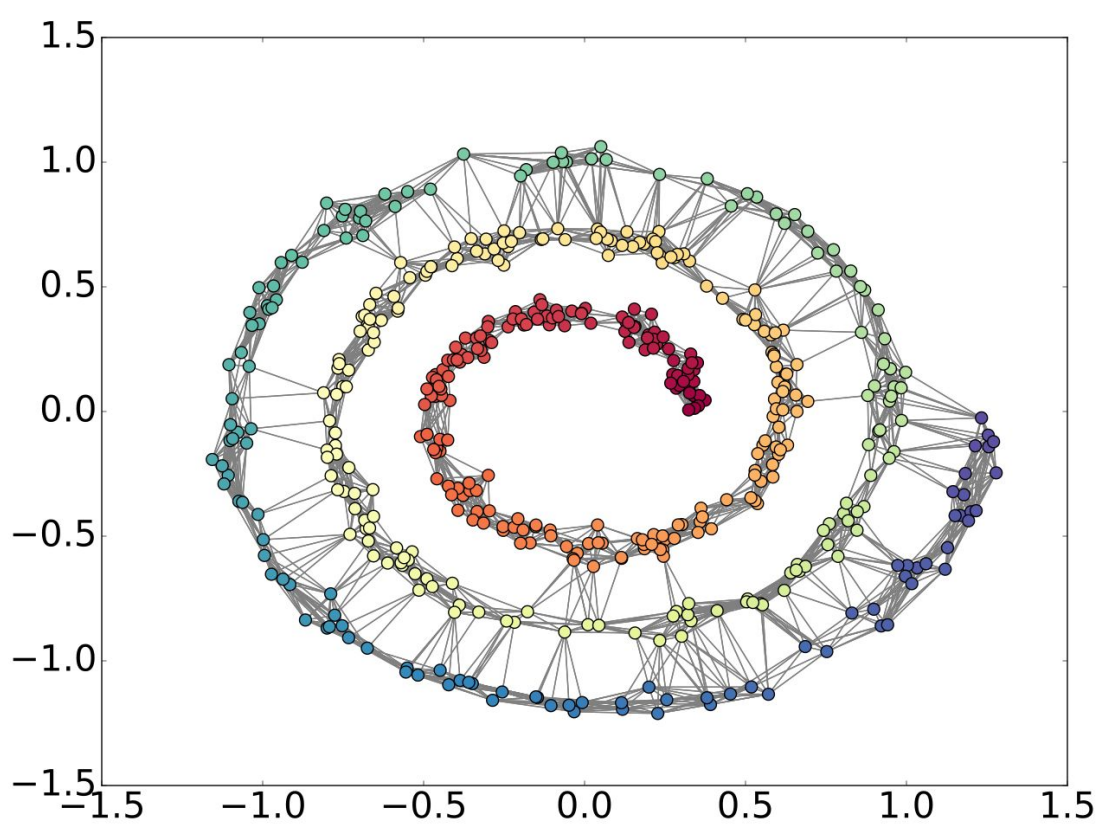




\section{Key Observations from Manifold Geometry}

- Red and blue points are from different regions of the manifold

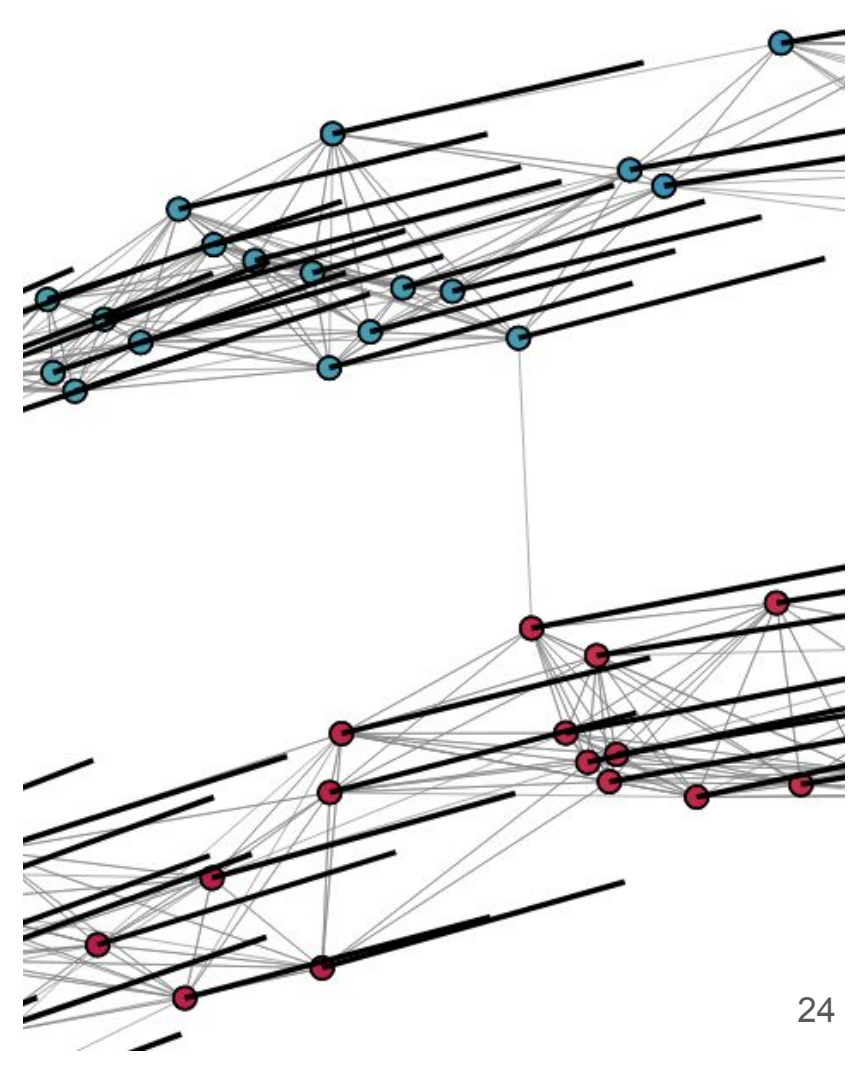




\section{Key Observations from Manifold Geometry}

- Red and blue points are from different regions of the manifold

- False edges disagree with the tangent spaces

- Tangent spaces (thick, black lines) tell us which edges to remove

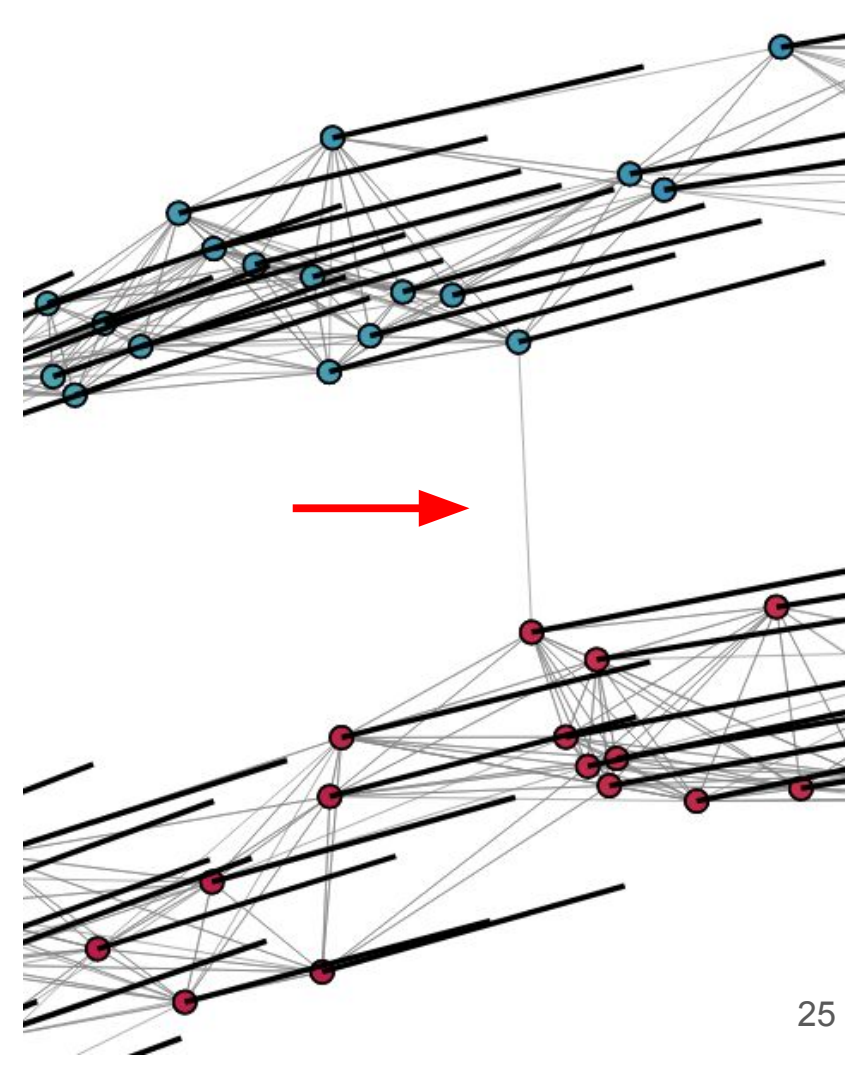




\section{Key Observations from Manifold Geometry}

- Red and blue points are from different regions of the manifold

- False edges disagree with the tangent spaces

- Tangent spaces (thick, black lines) tell us which edges to remove

- Problem: poor local neighborhoods skew tangent space estimates

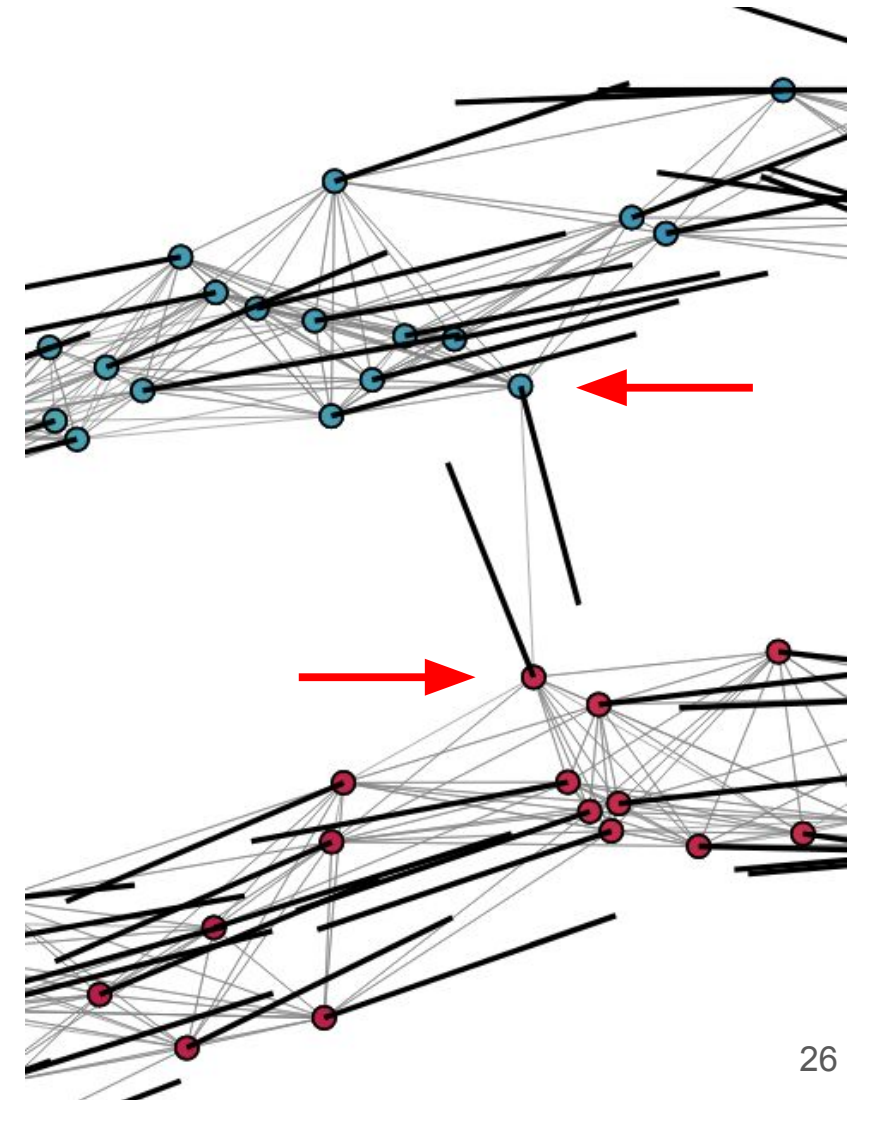




\section{Key Observations from Manifold Geometry}

- Red and blue points are from different regions of the manifold

- False edges disagree with the tangent spaces

- Tangent spaces (thick, black lines) tell us which edges to remove

- Problem: poor local neighborhoods skew tangent space estimates

- Nearby points have similar tangent spaces - Can we use this to obtain a better estimate?

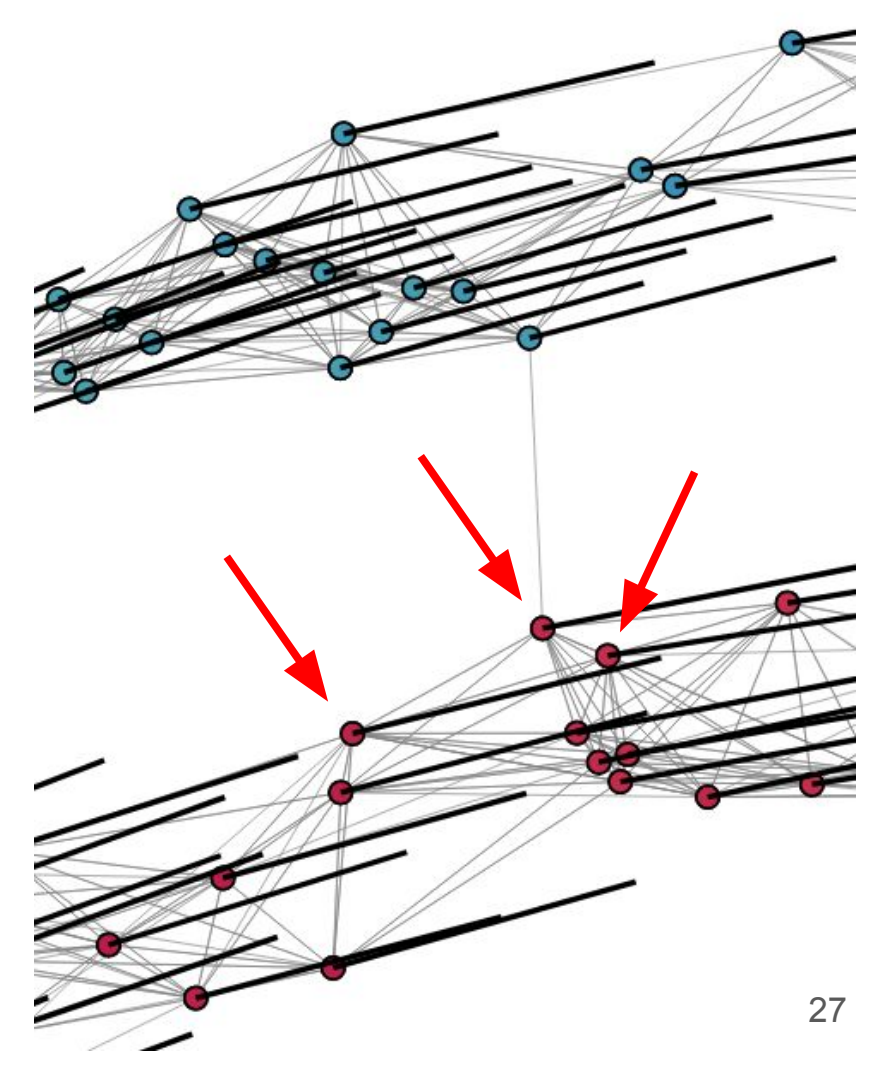




\section{Tangent Space Estimation via Belief Propagation}

- Formulate as inference on a Markov Random Field (MRF)

- For each point

- Latent variable represents its tangent space

- Observation from PCA on local neighborhood

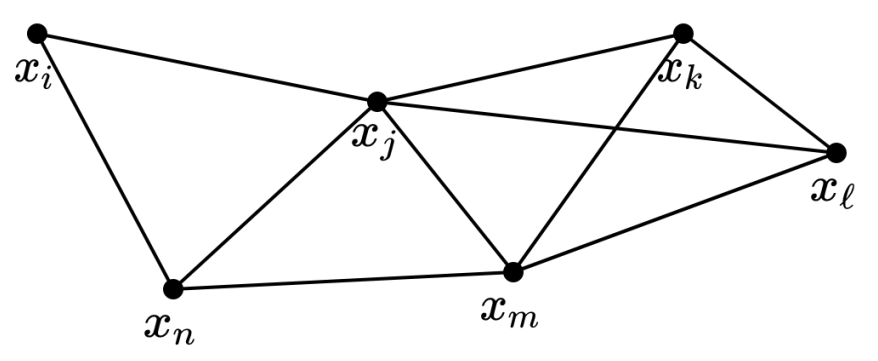

Neighborhood Graph

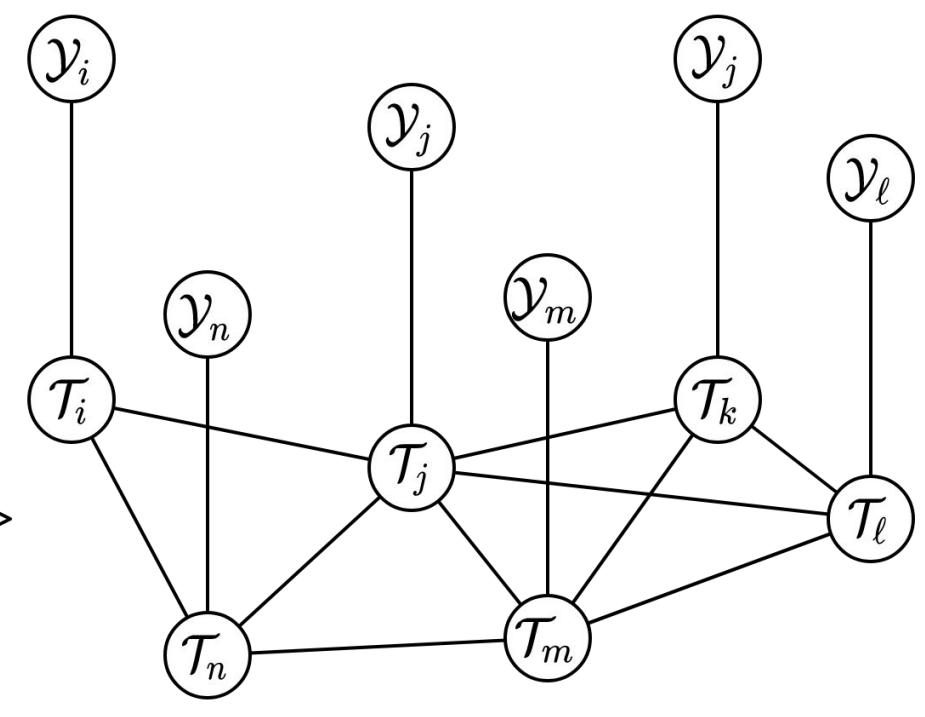

MRF 


\section{Spiral Experiment}

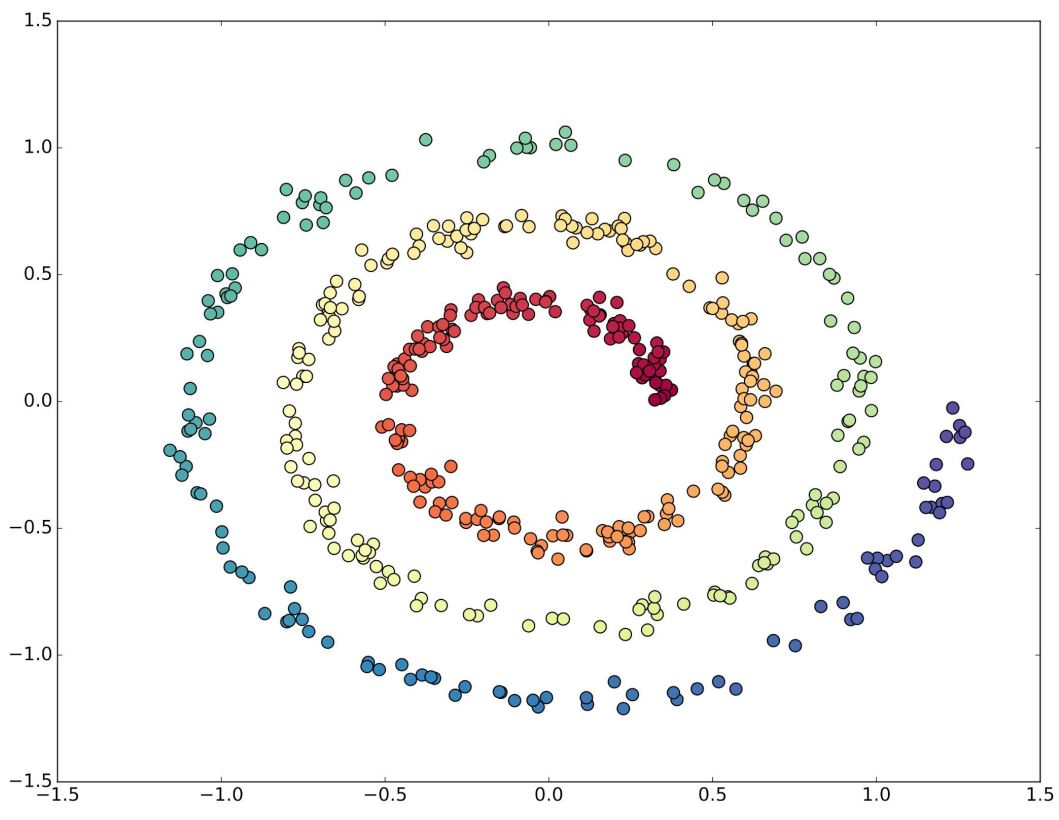

Dataset

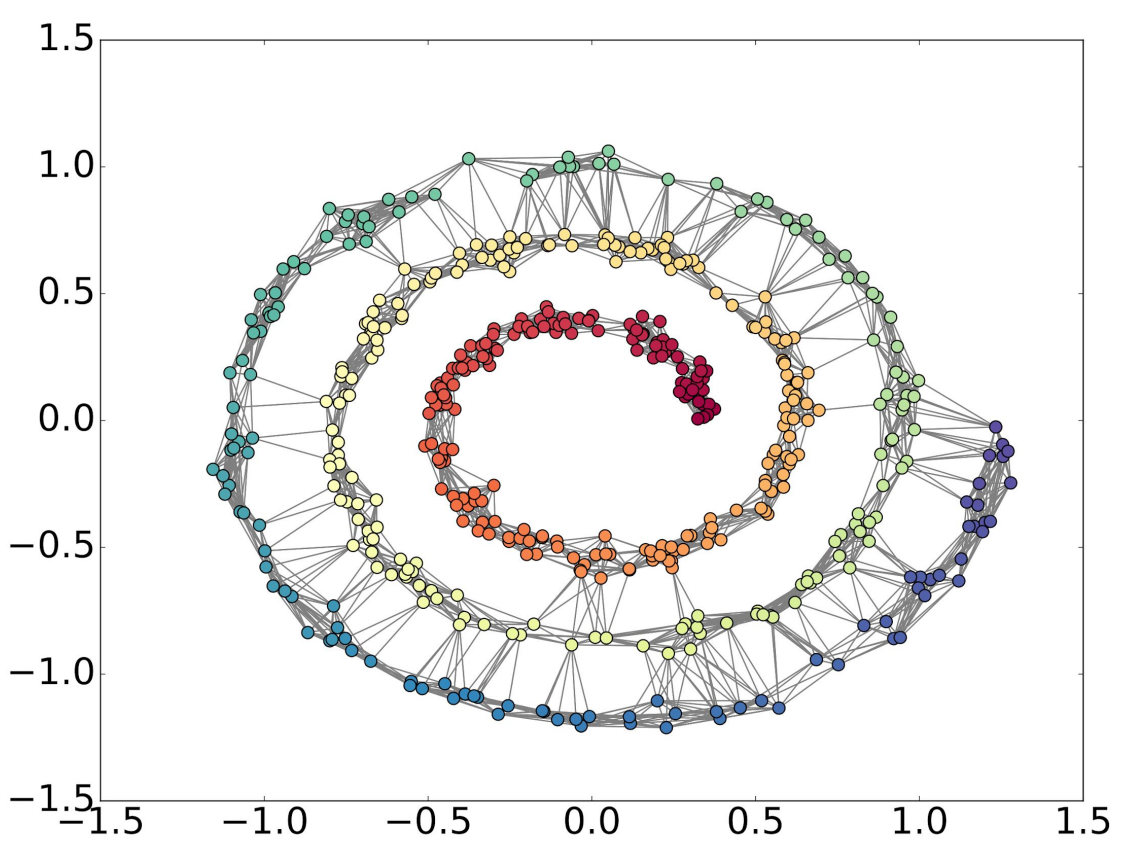

$k$-Nearest Neighbors 


\section{Spiral Experiment Tangent Estimation}
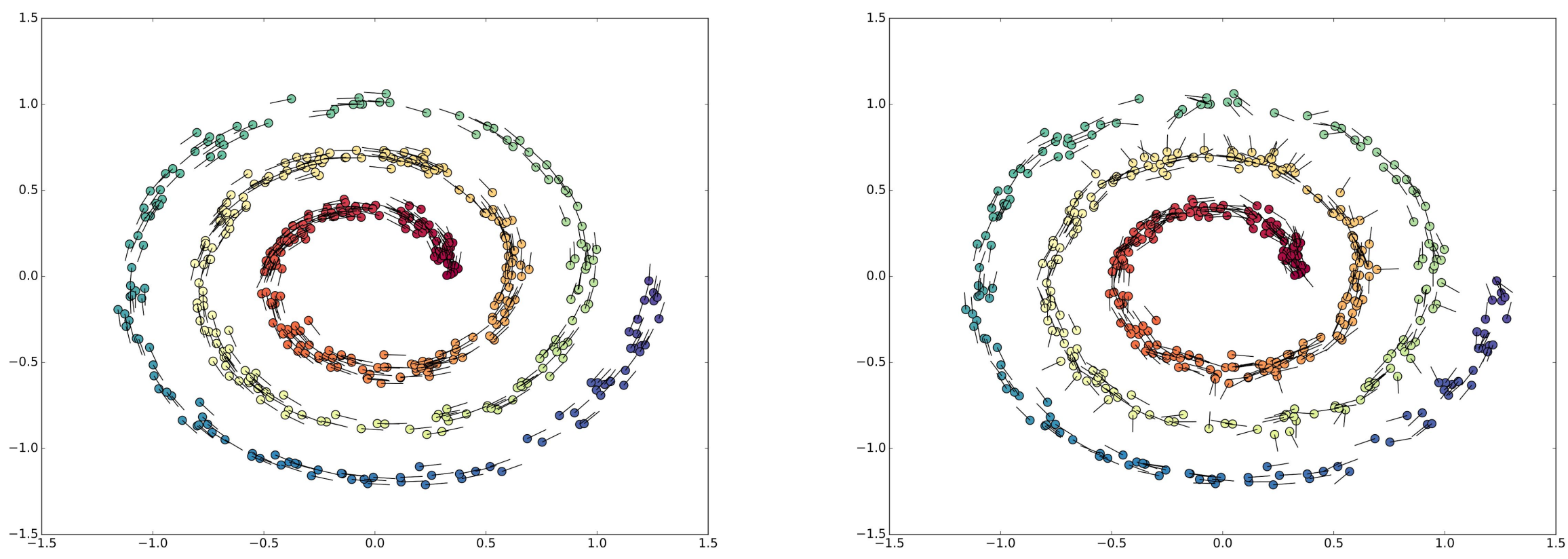

Ground Truth

PCA 


\section{Spiral Experiment Tangent Estimation}
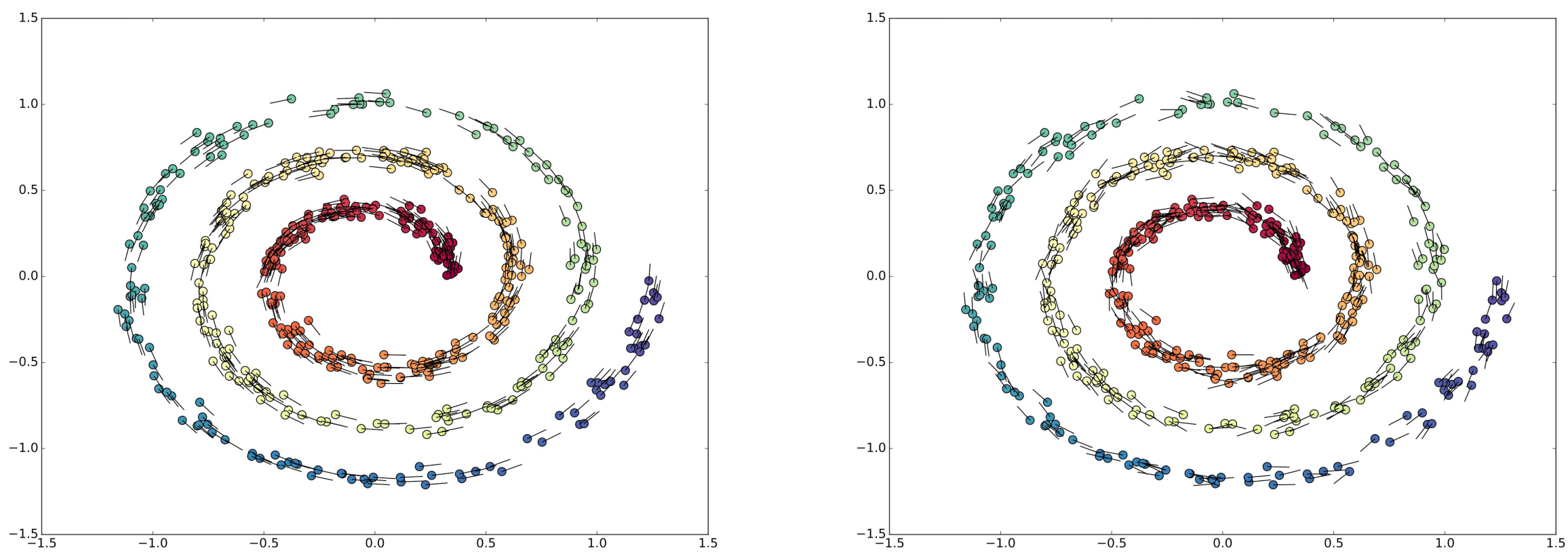

Ground Truth

TSBP 


\section{Spiral Experiment Nearest Neighbors}

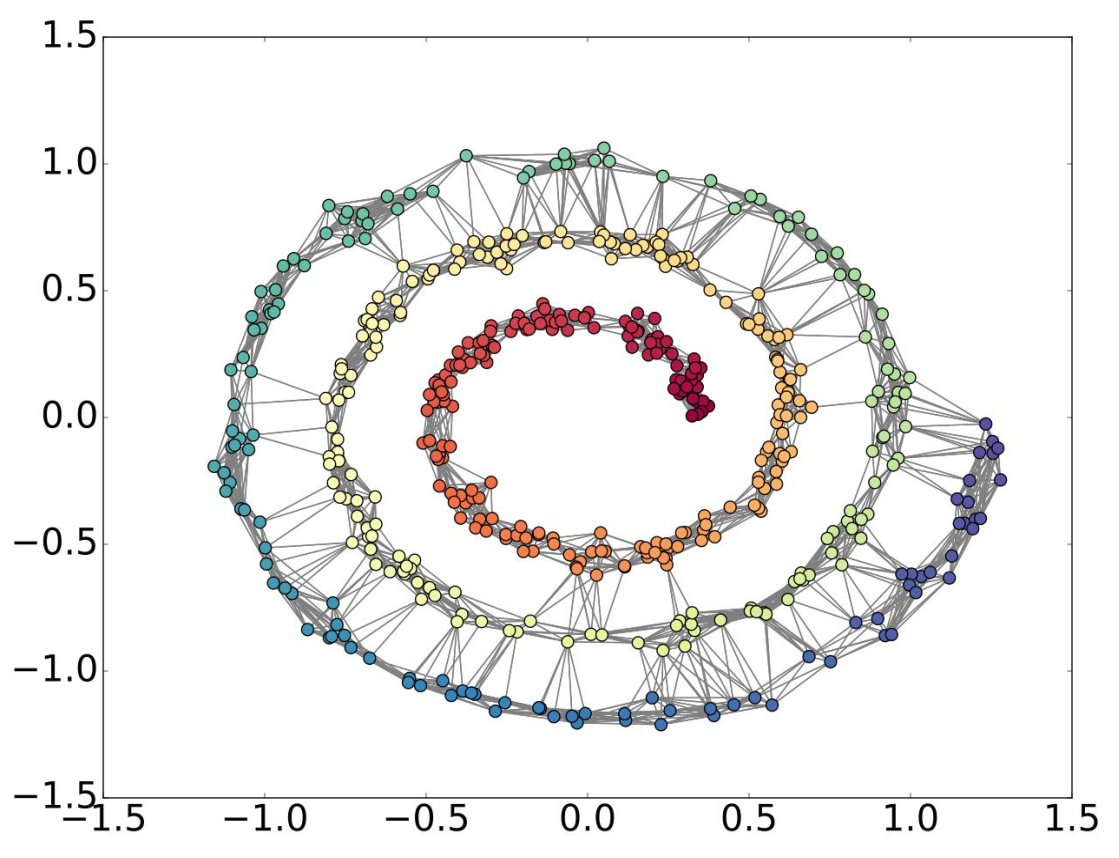

$k$-Nearest Neighbors

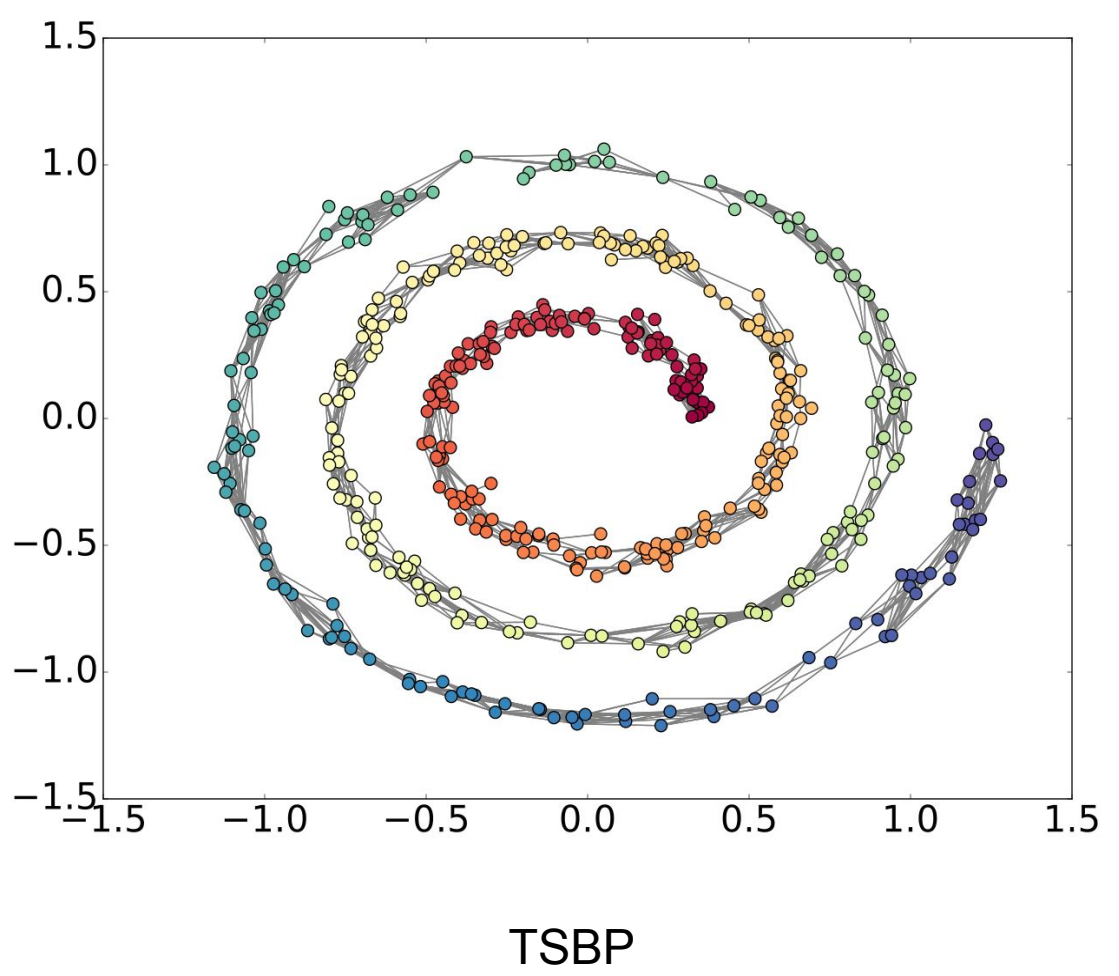




\section{Spiral Experiment Belief Propagation}




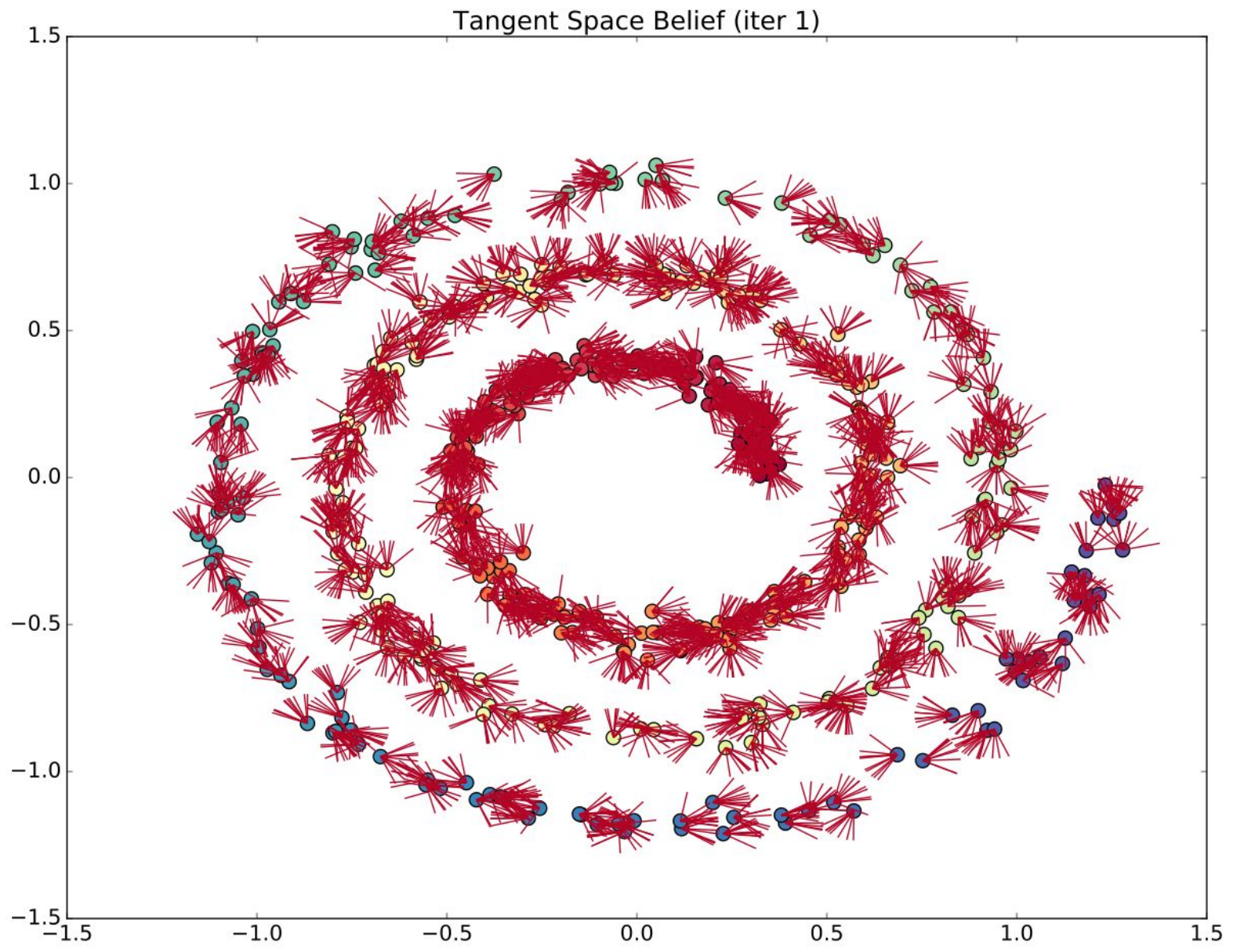




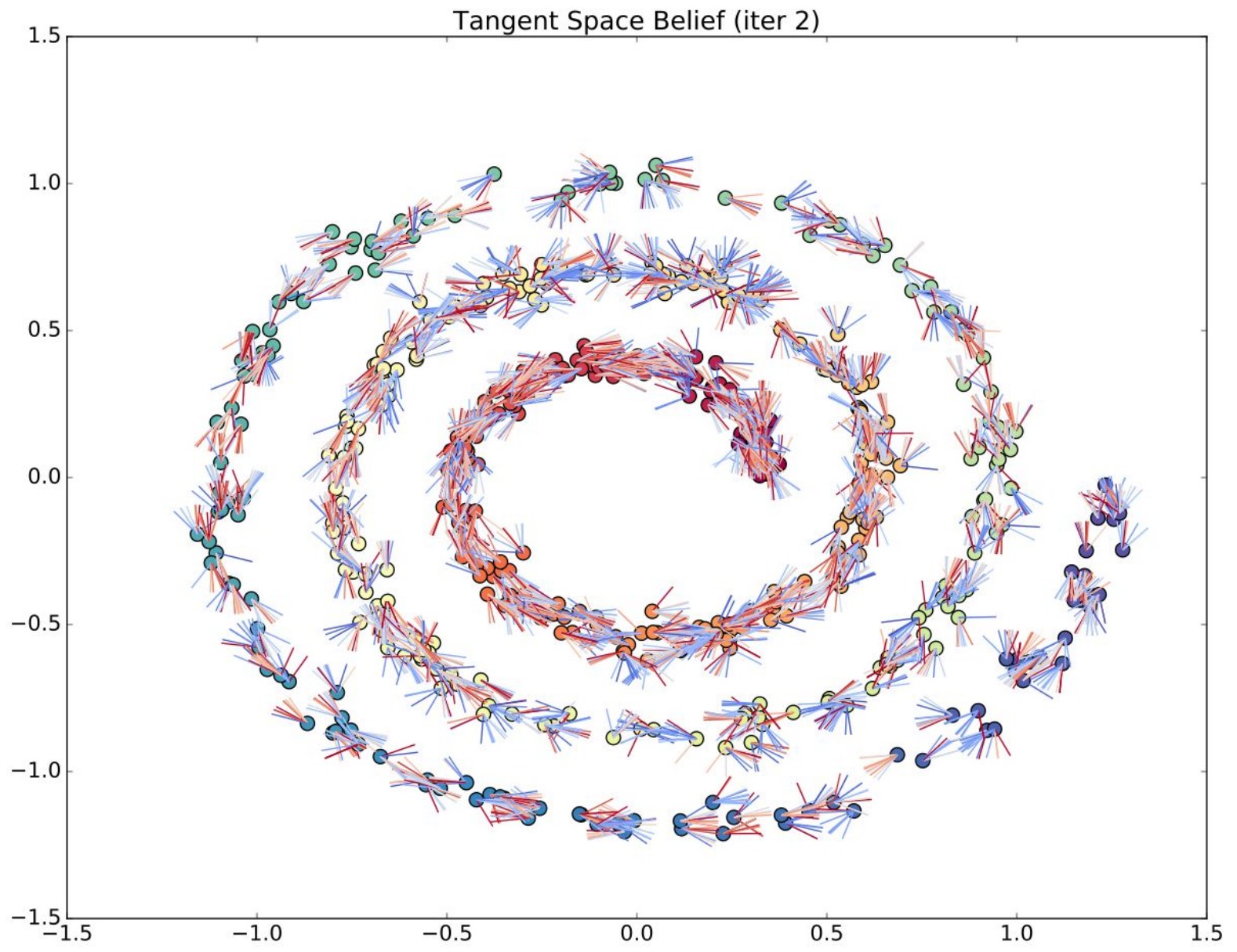




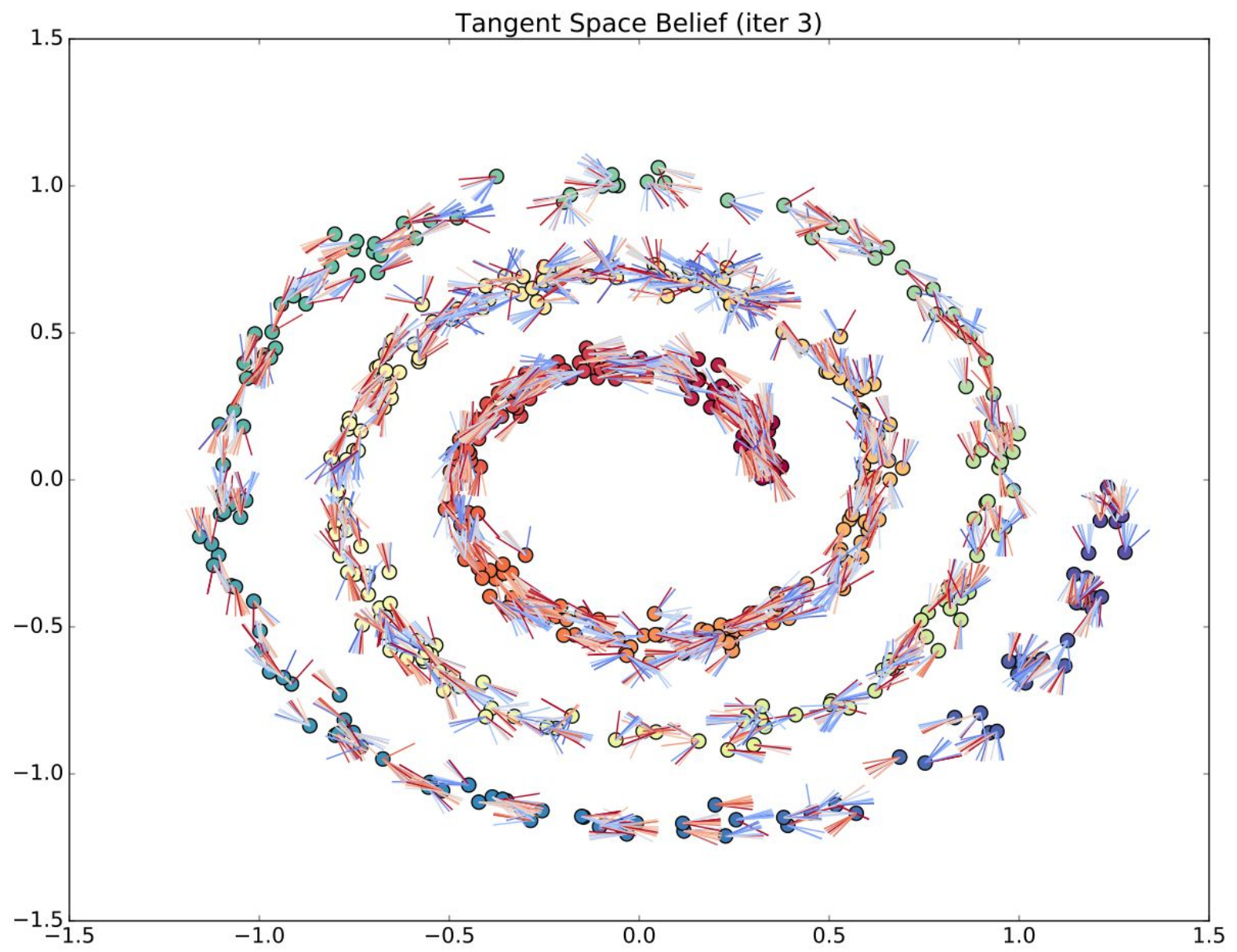




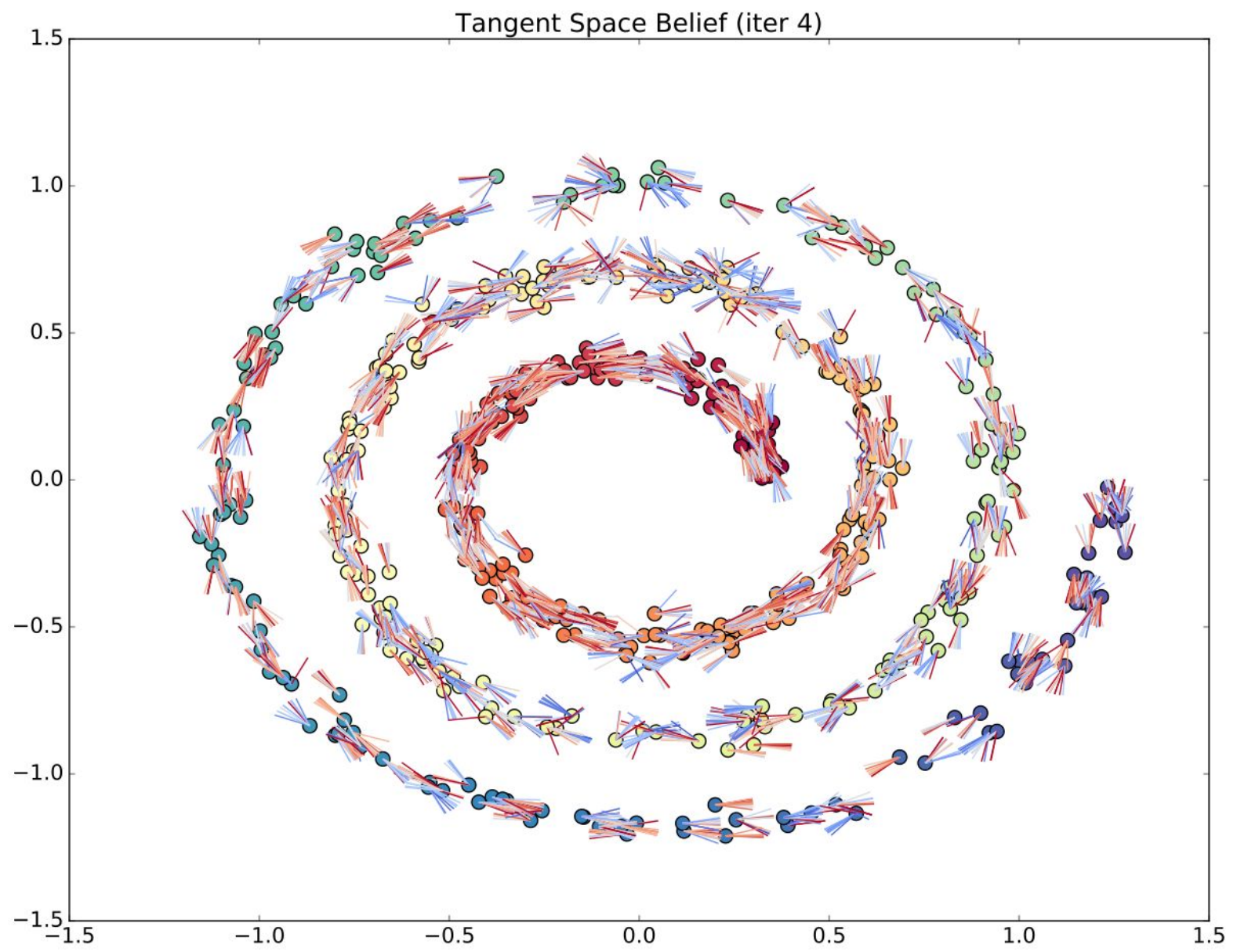




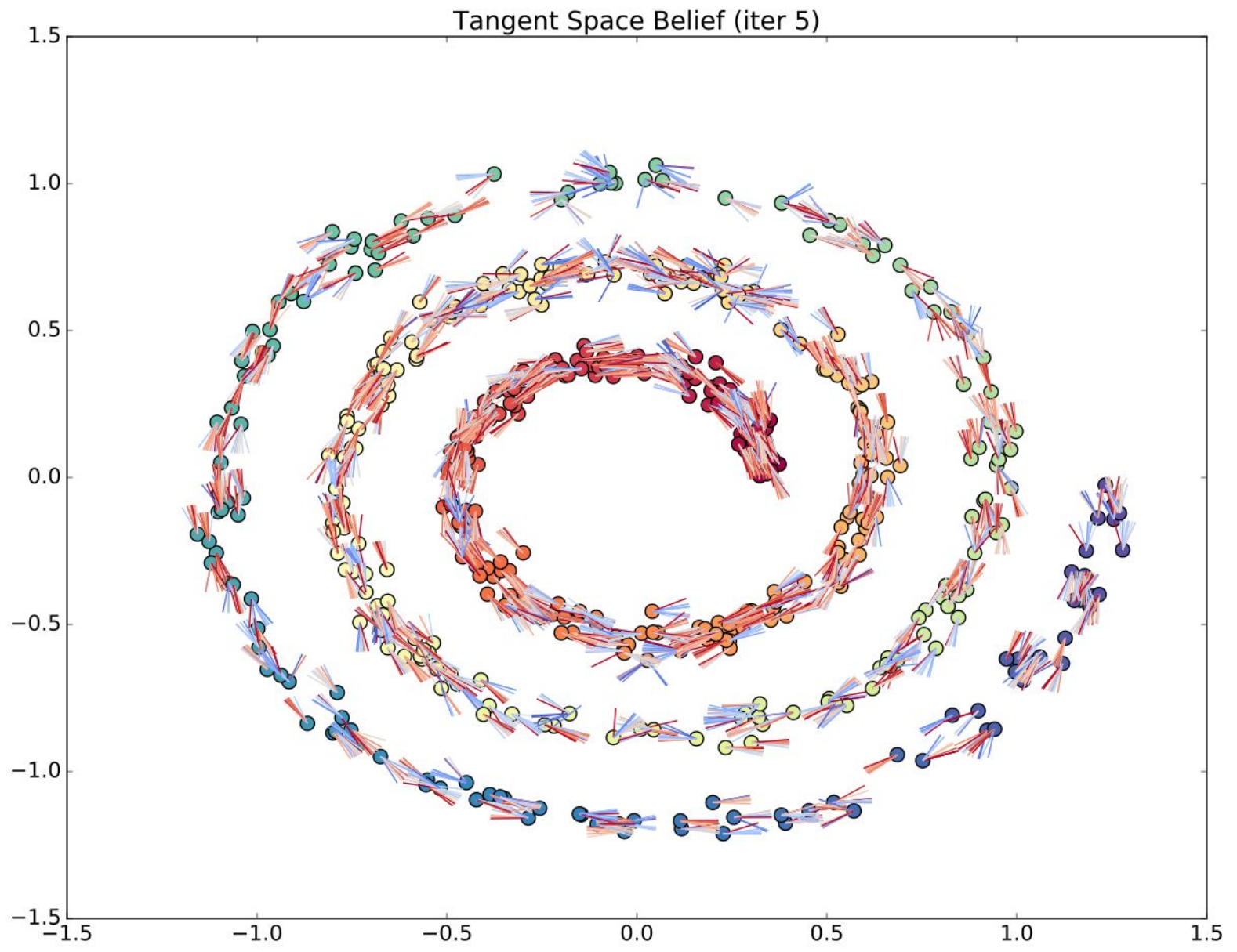




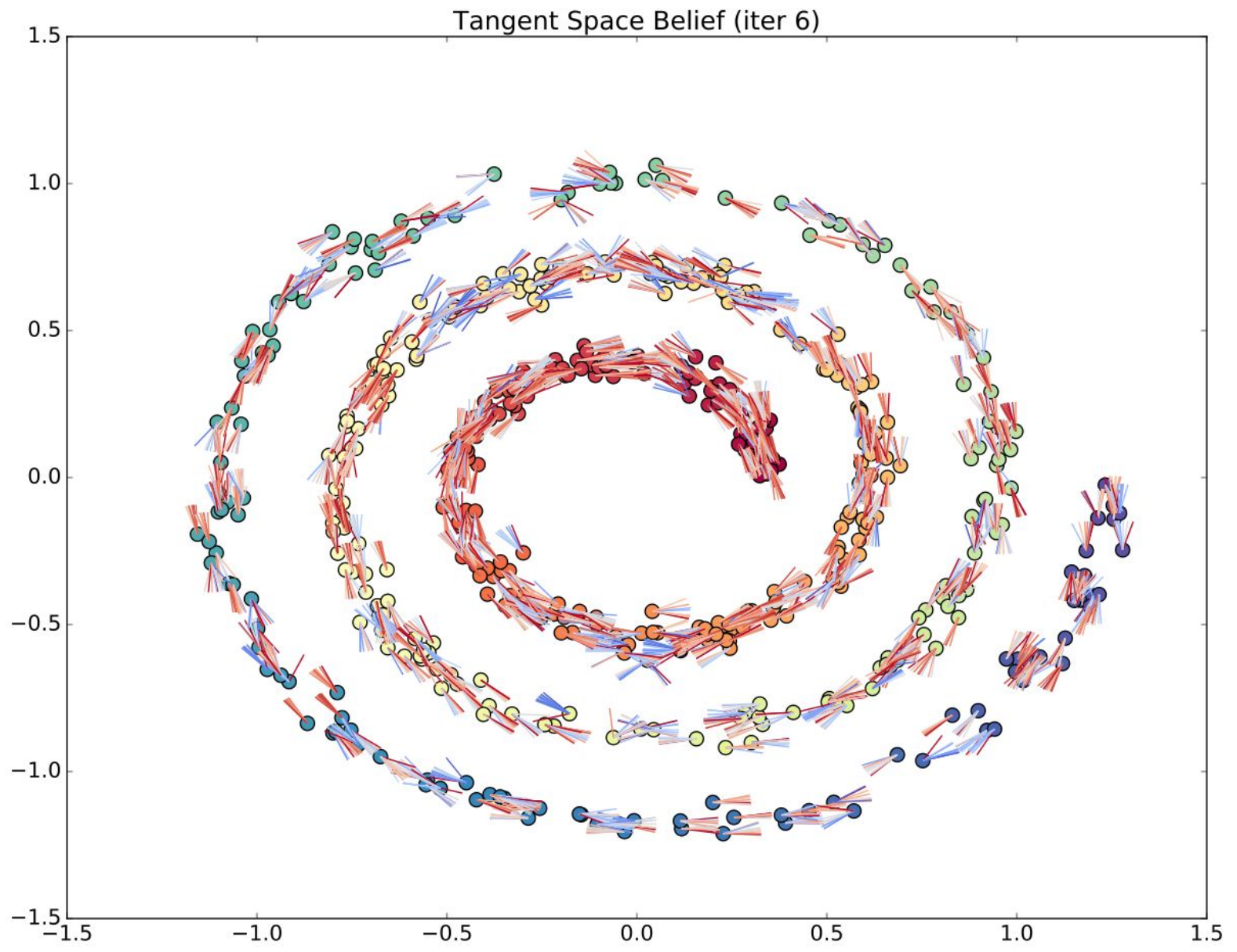




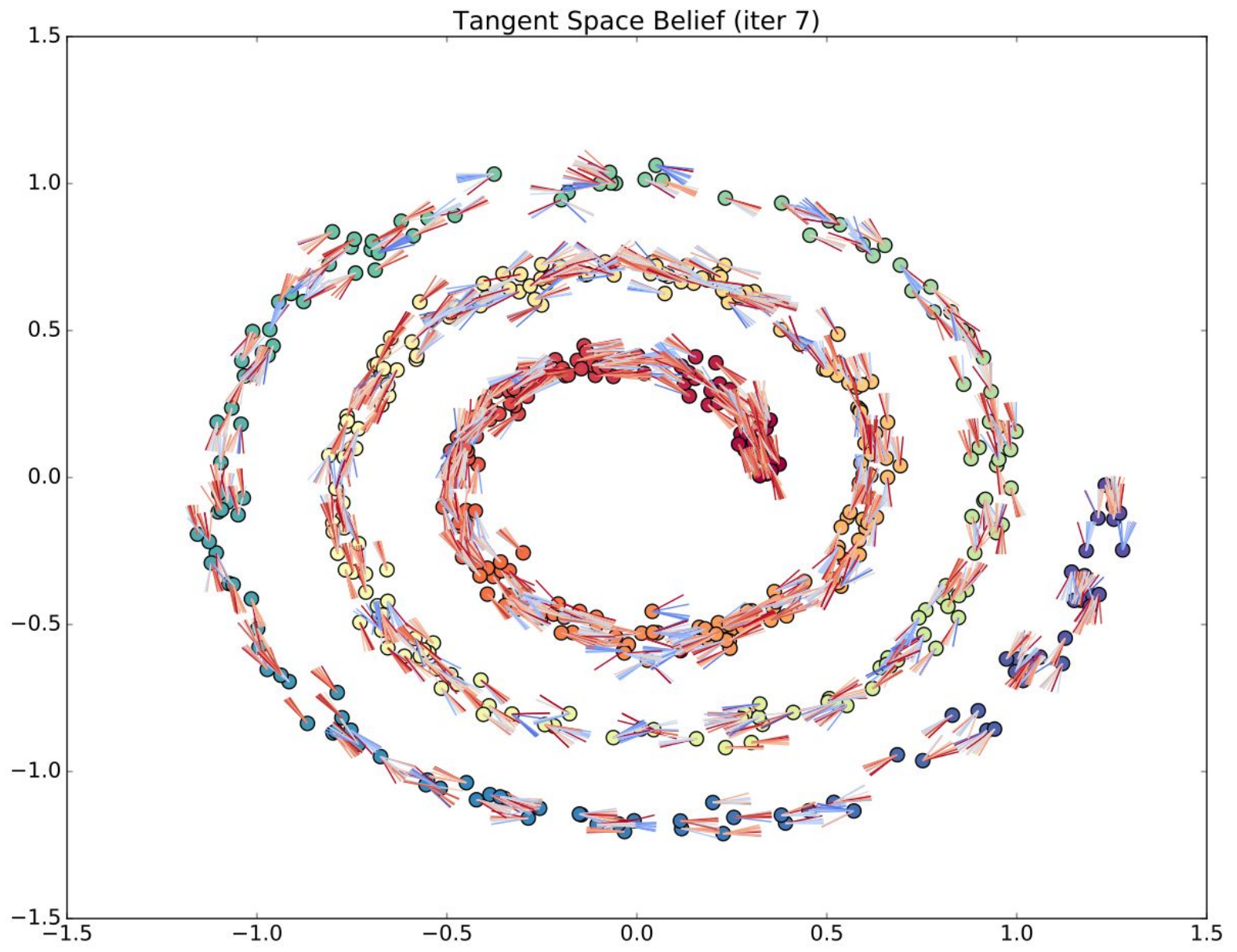




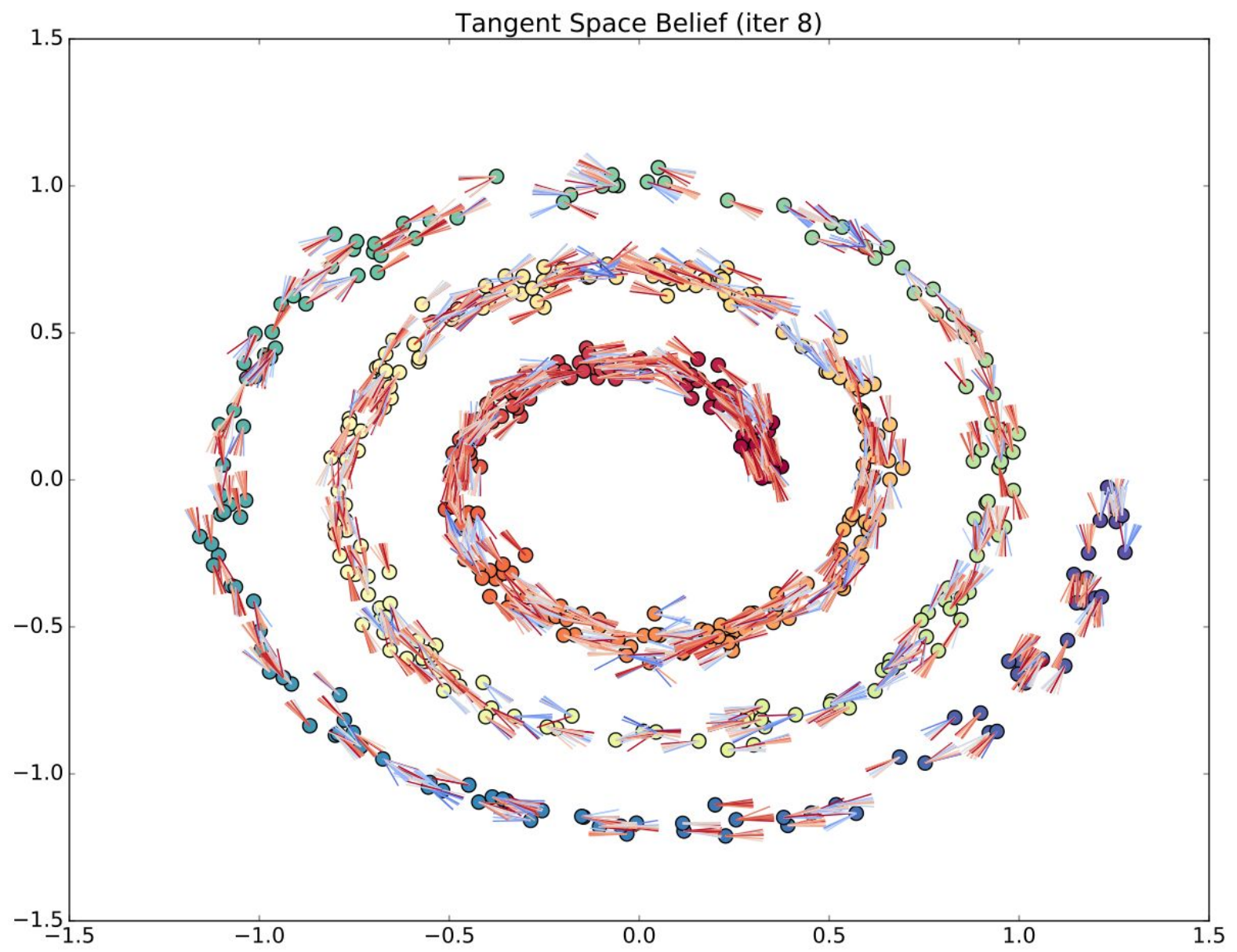




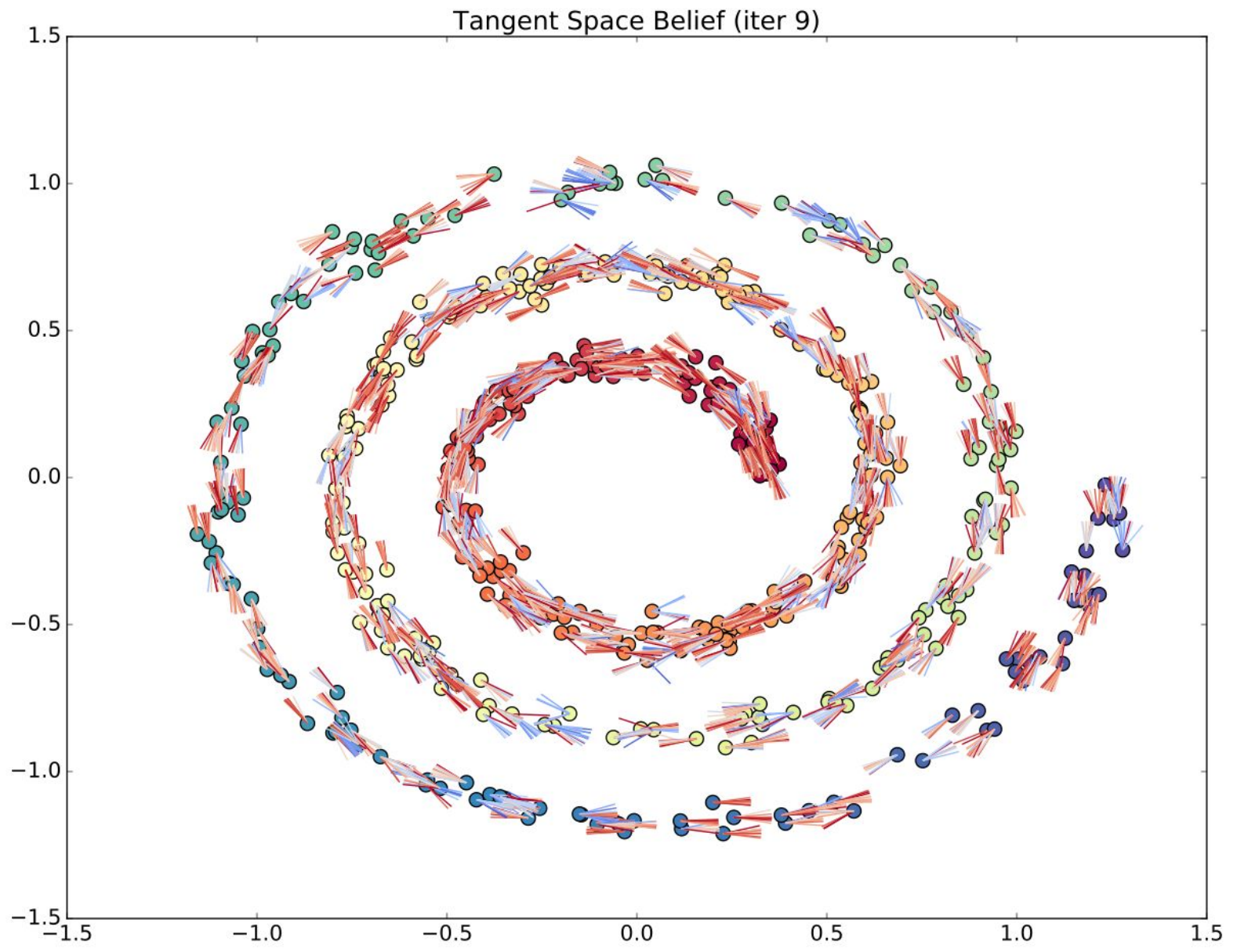




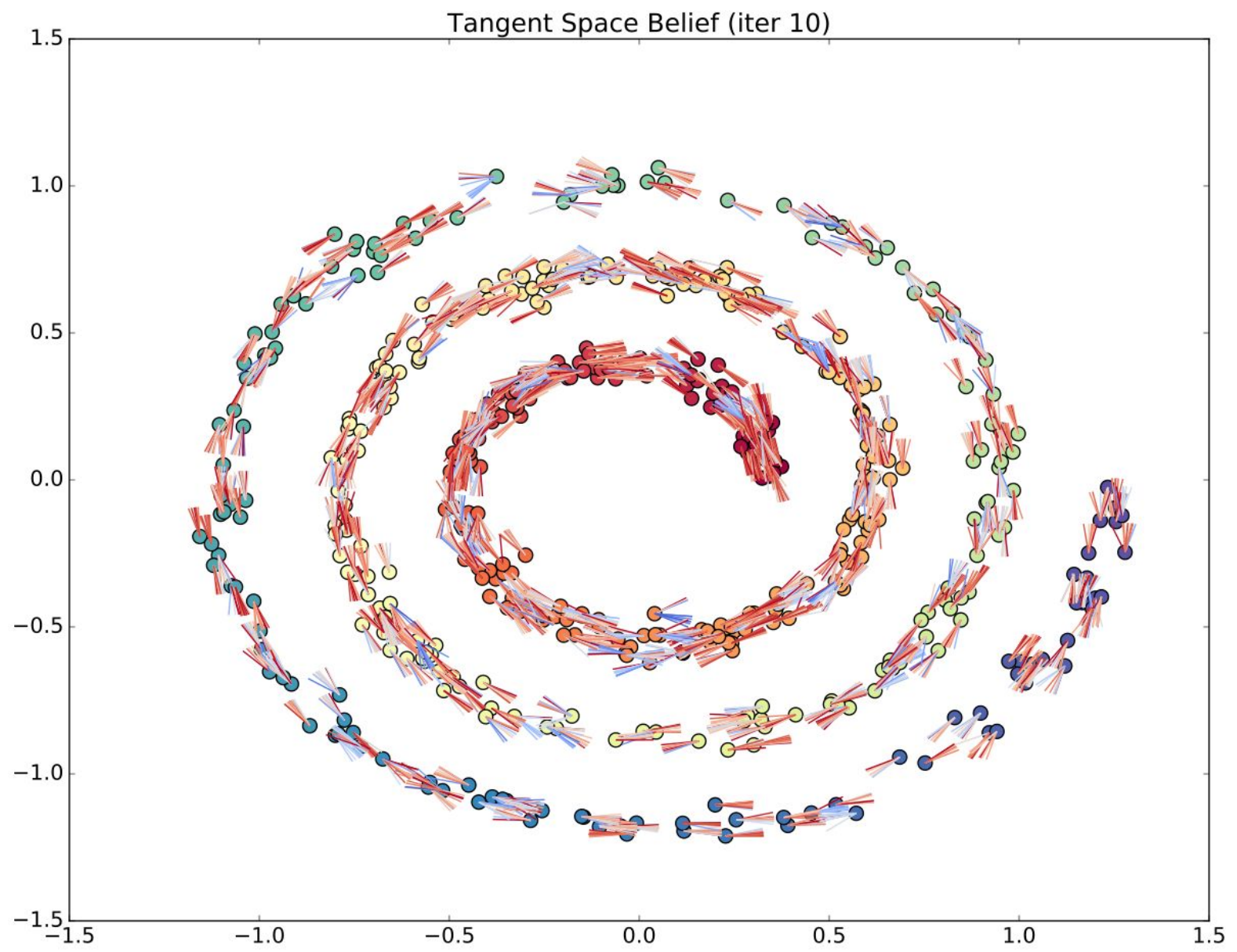




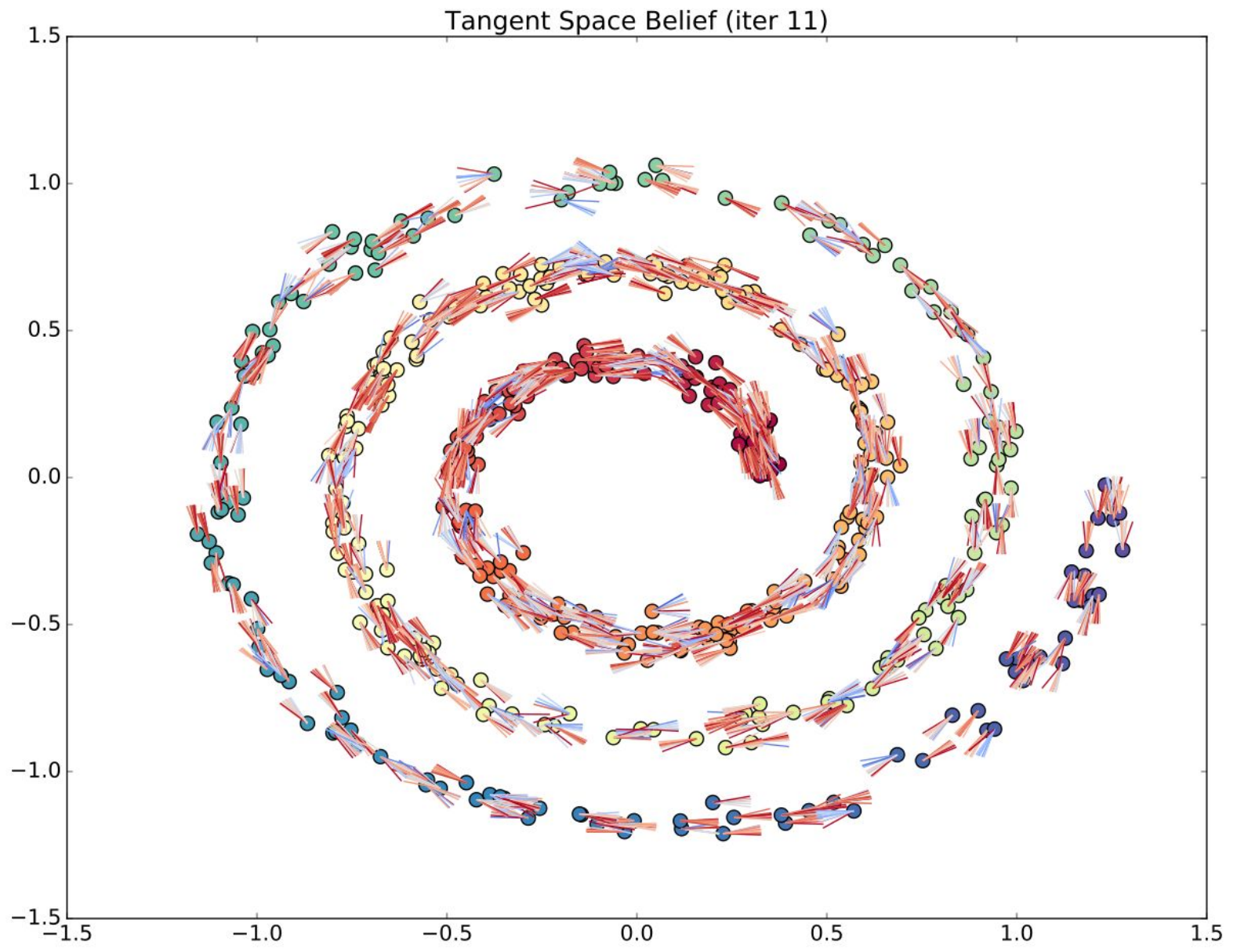




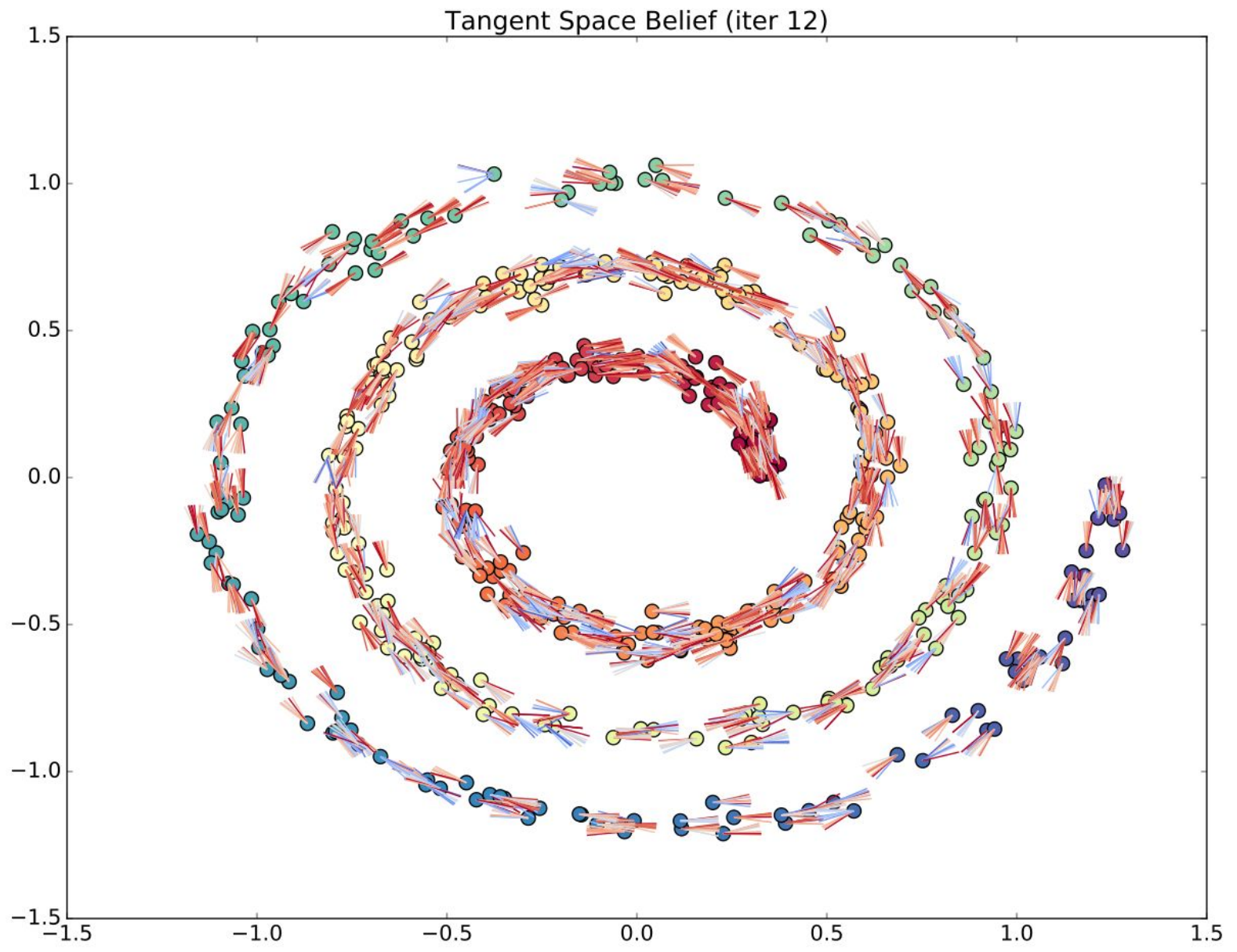




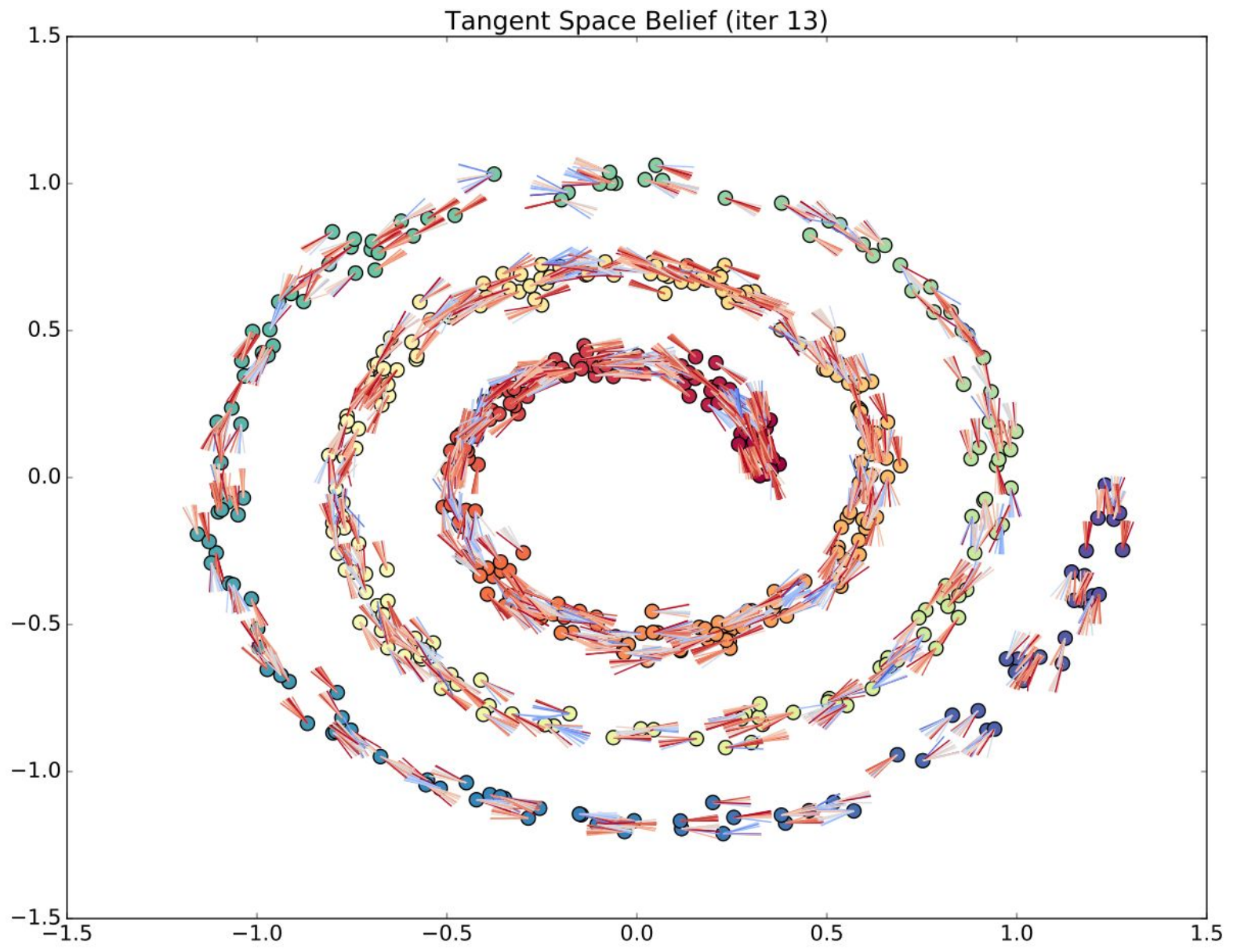




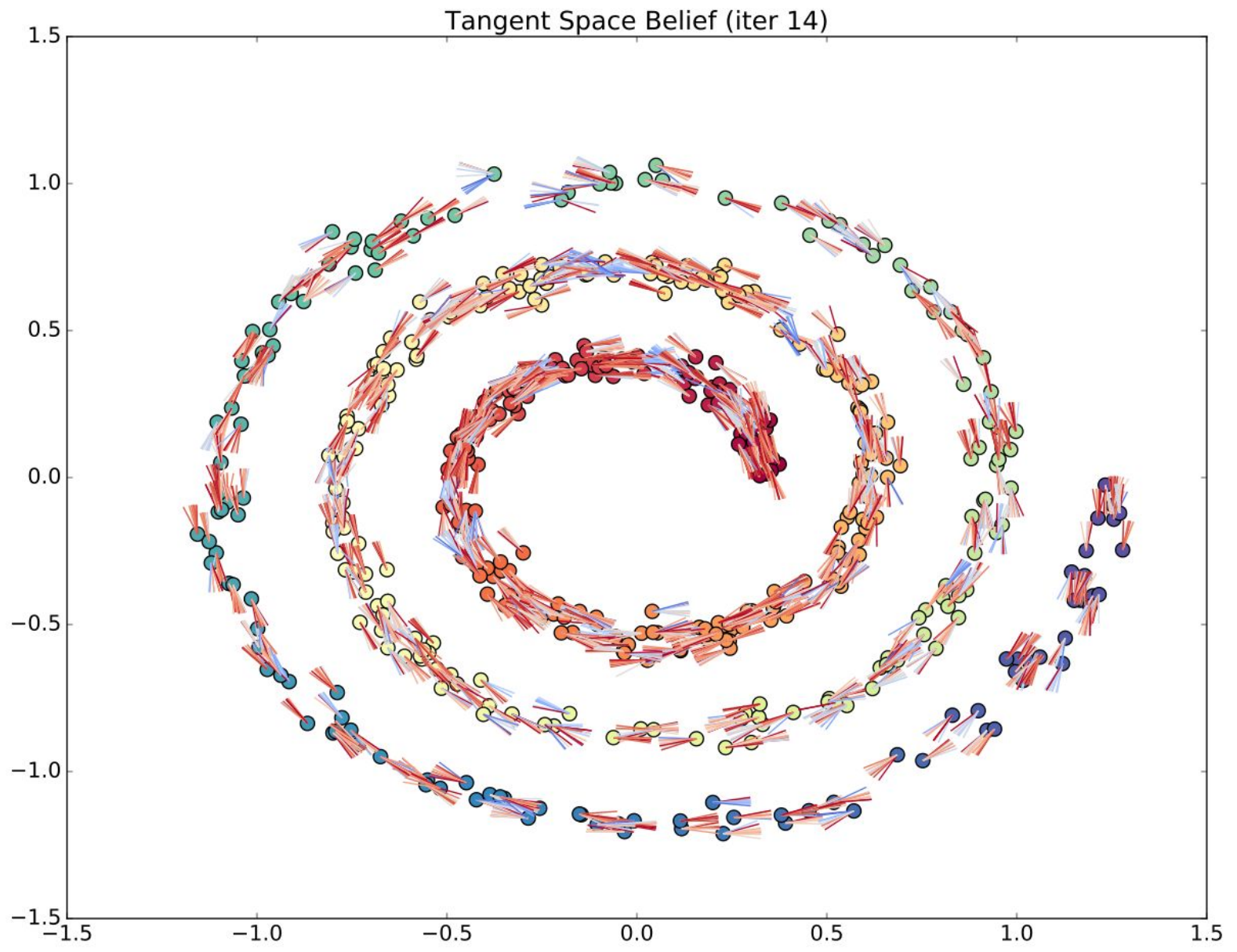




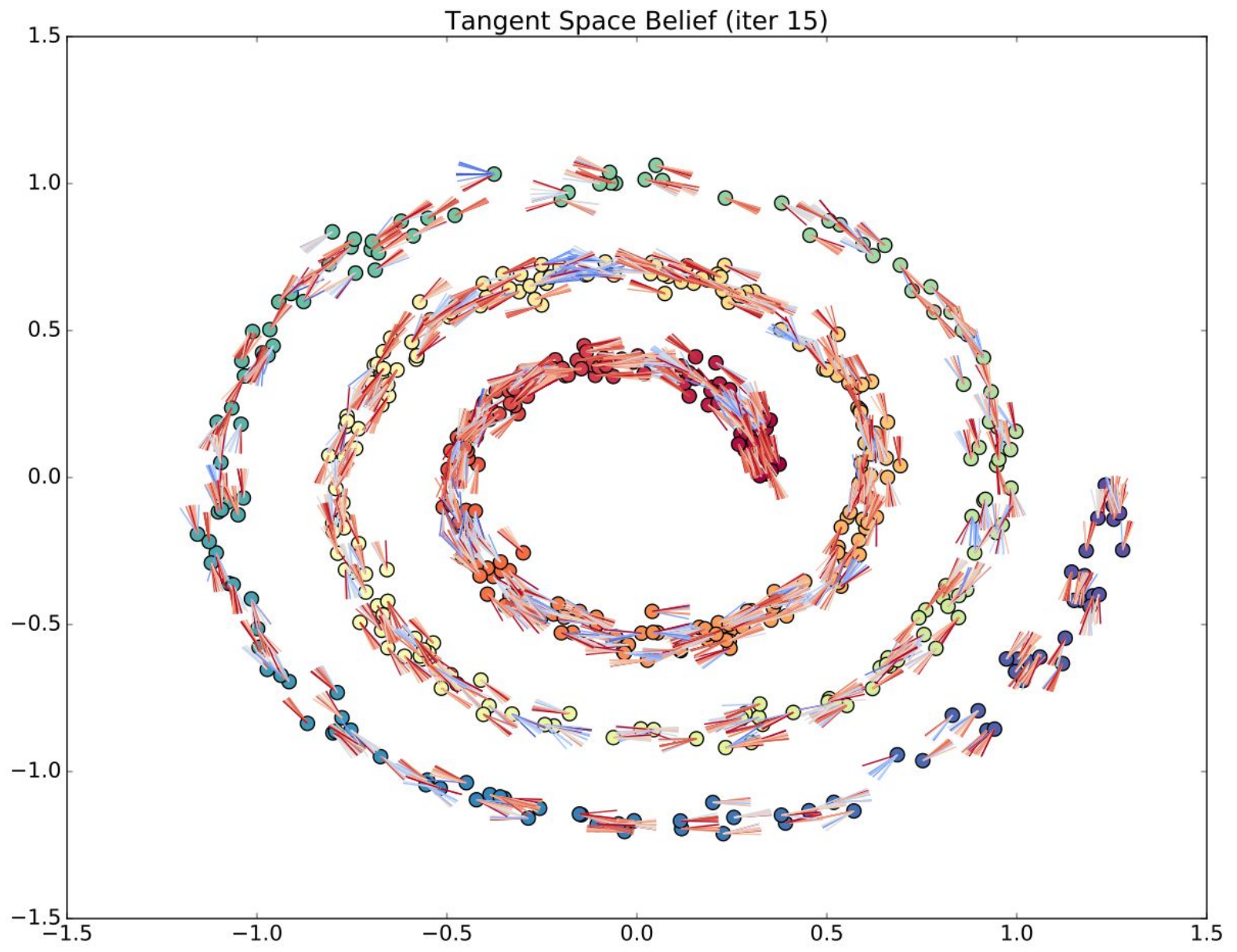




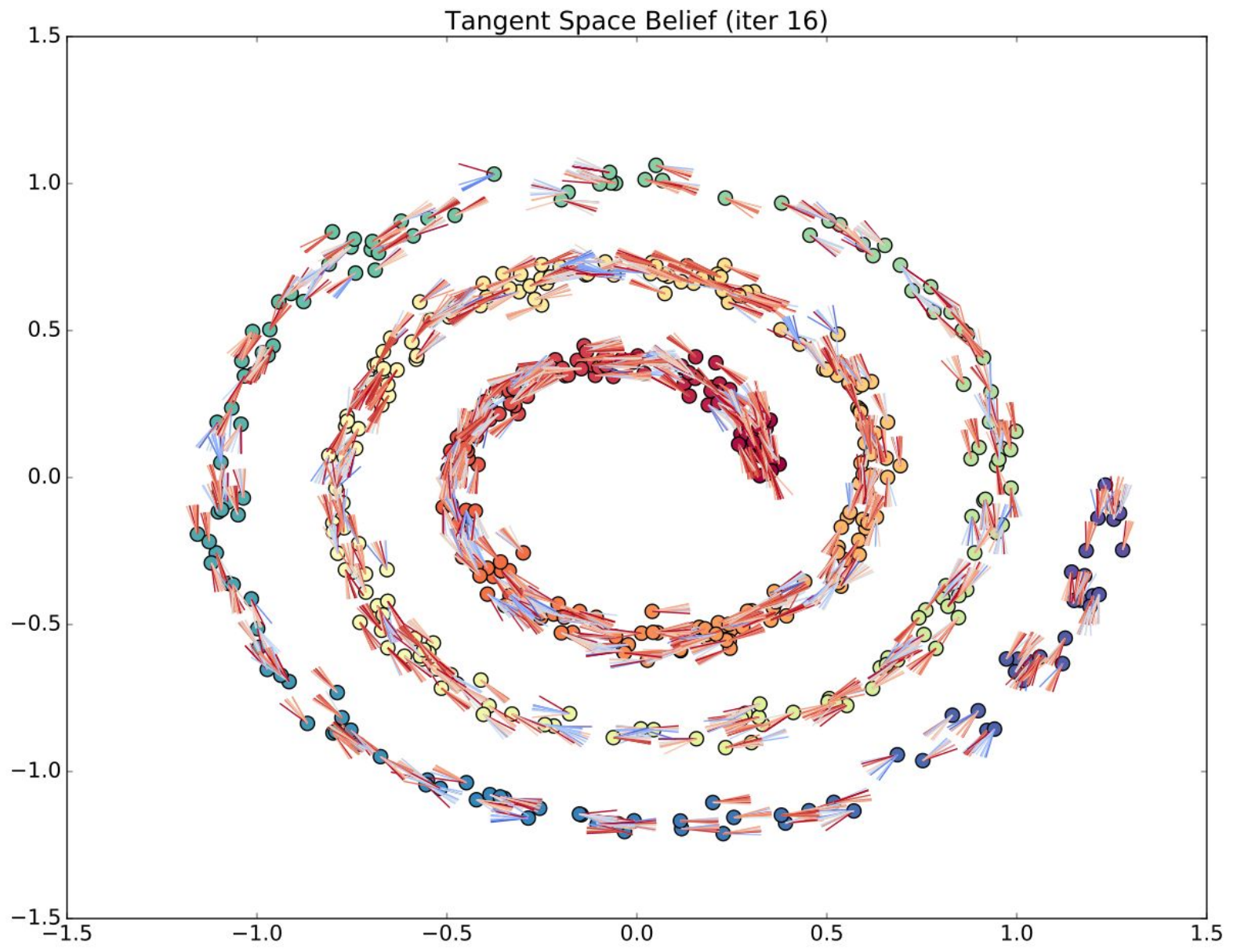




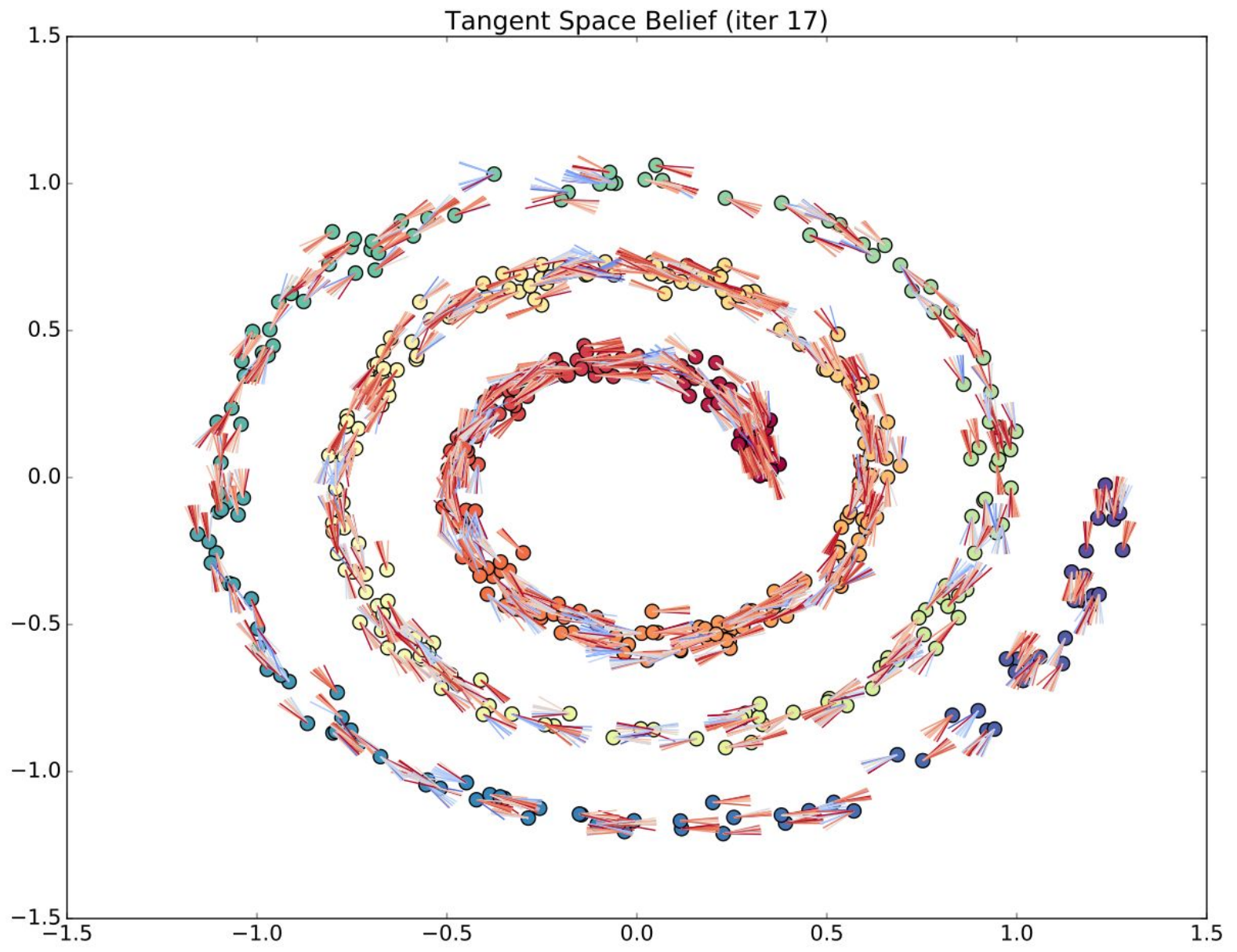




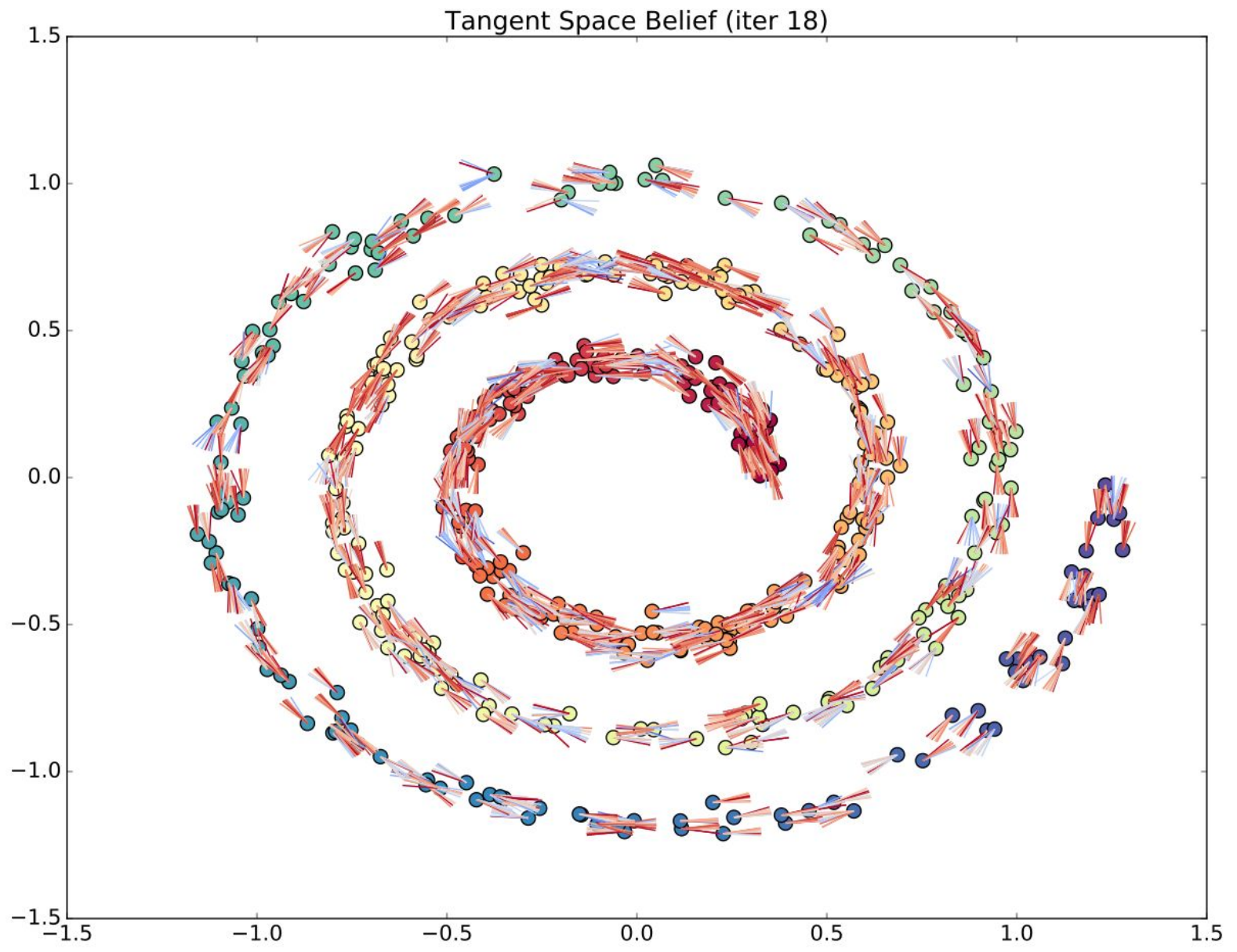




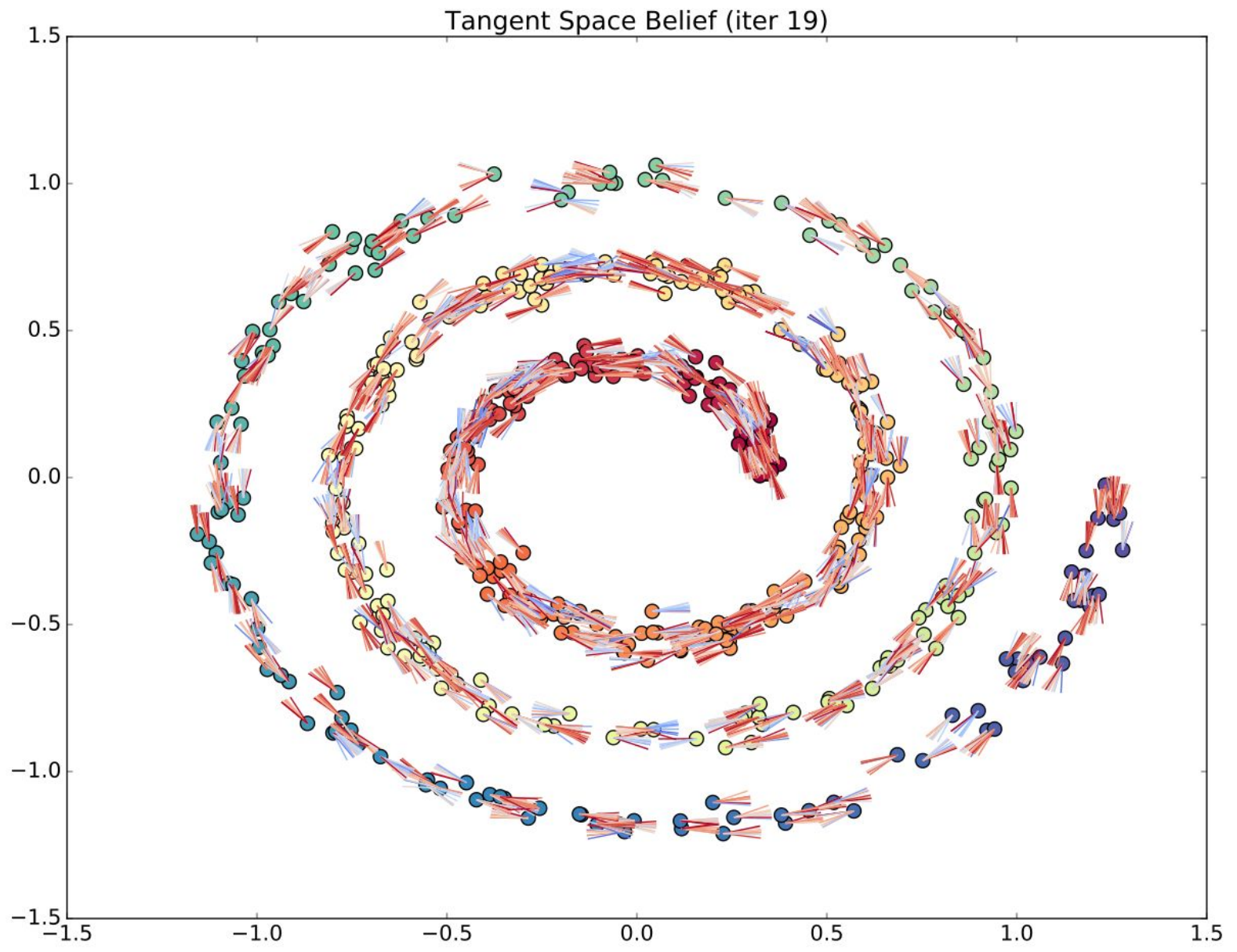




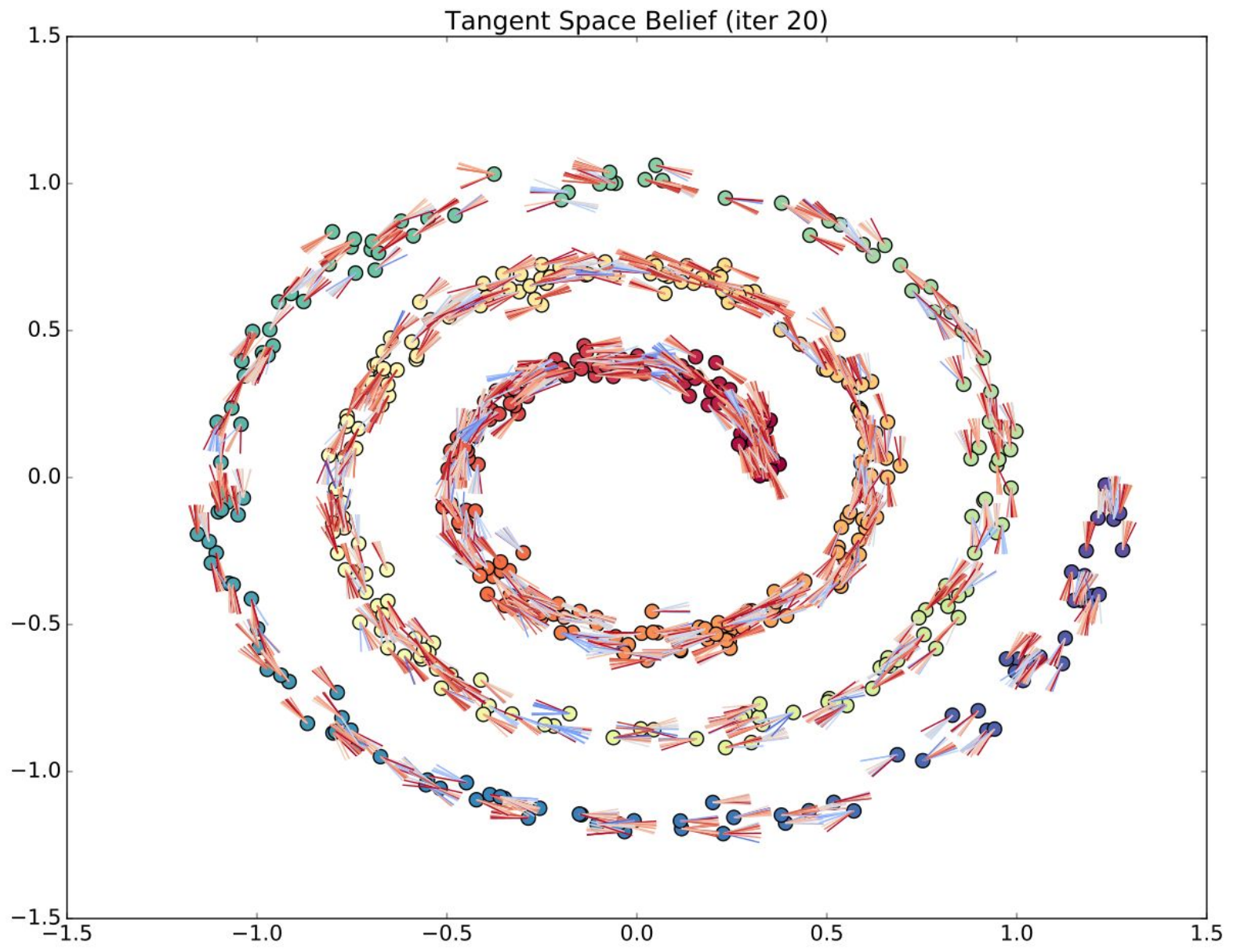




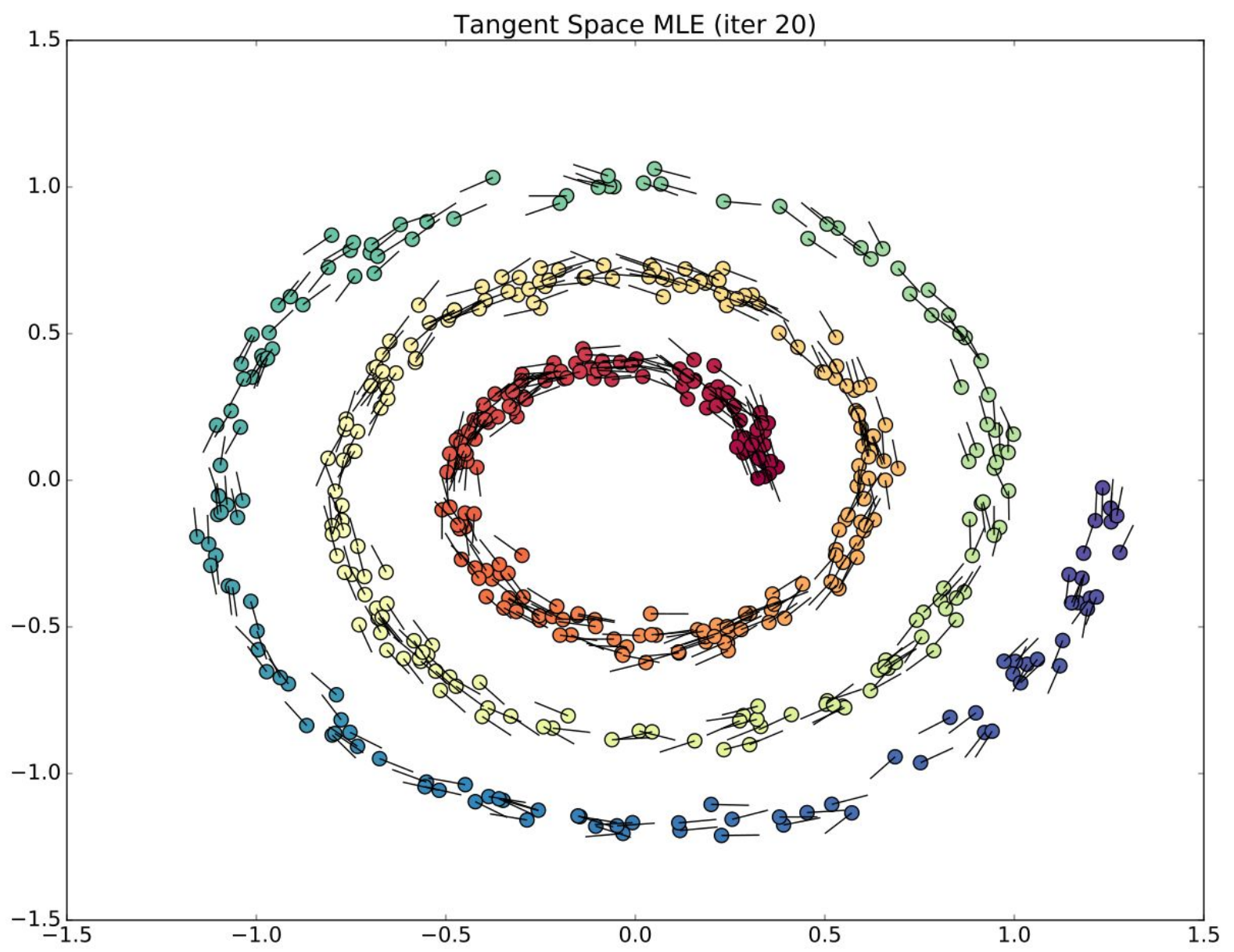




\section{Spiral Experiment Error}
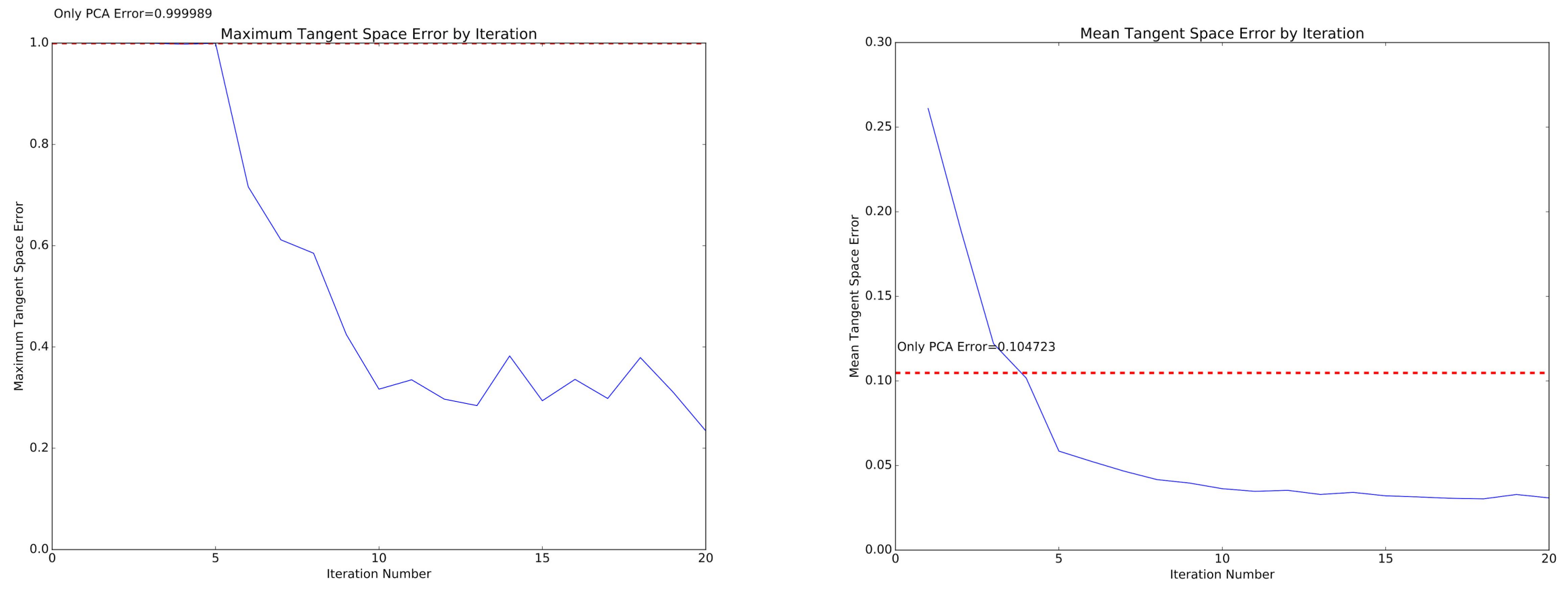


\section{Embedding Evaluation}

- Plot the original data parameters versus the embedding

- Compare nearest-neighbors and TSBP

- Expect a continuous, monotonic relationship

- Ideal embedding:
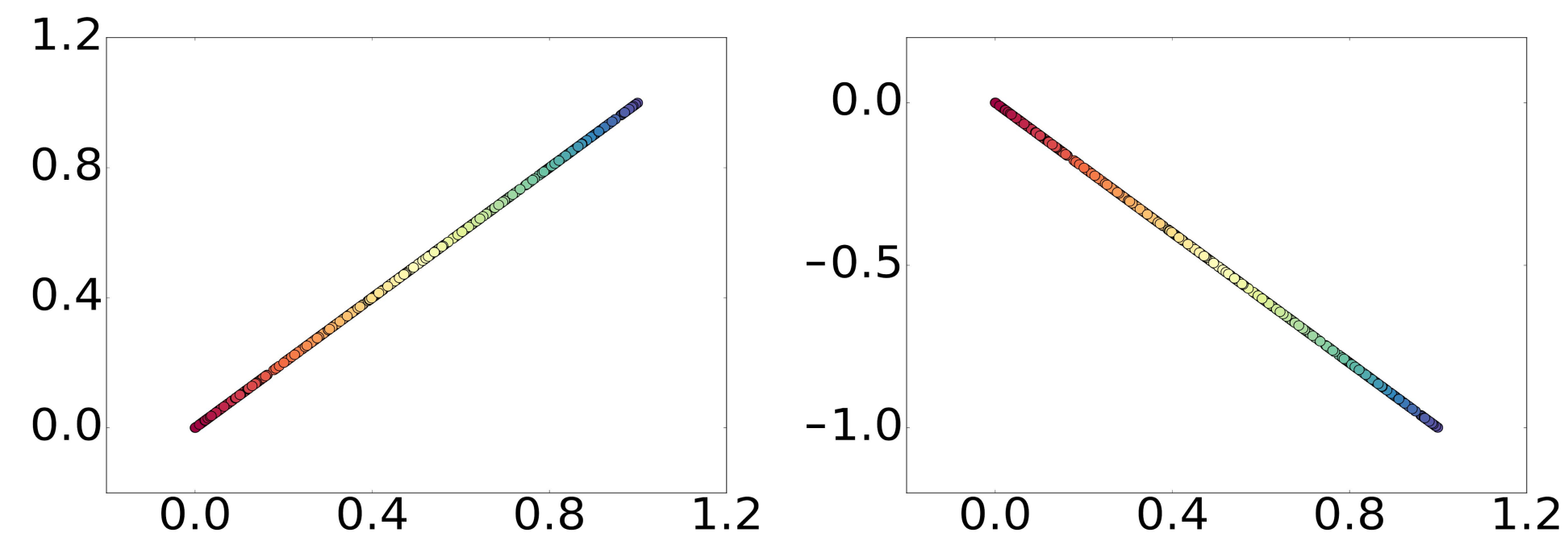


\section{Embedding Evaluation}
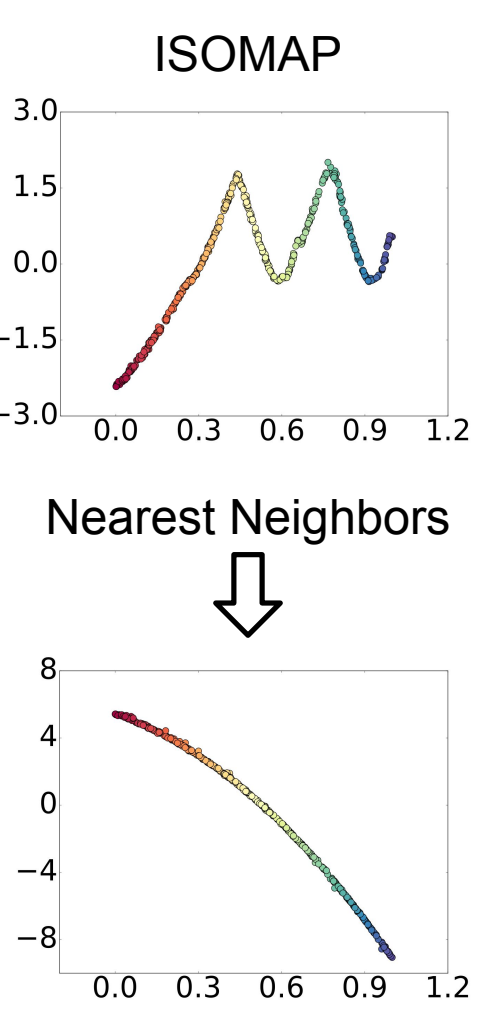

TSBP 


\section{Embedding Evaluation}
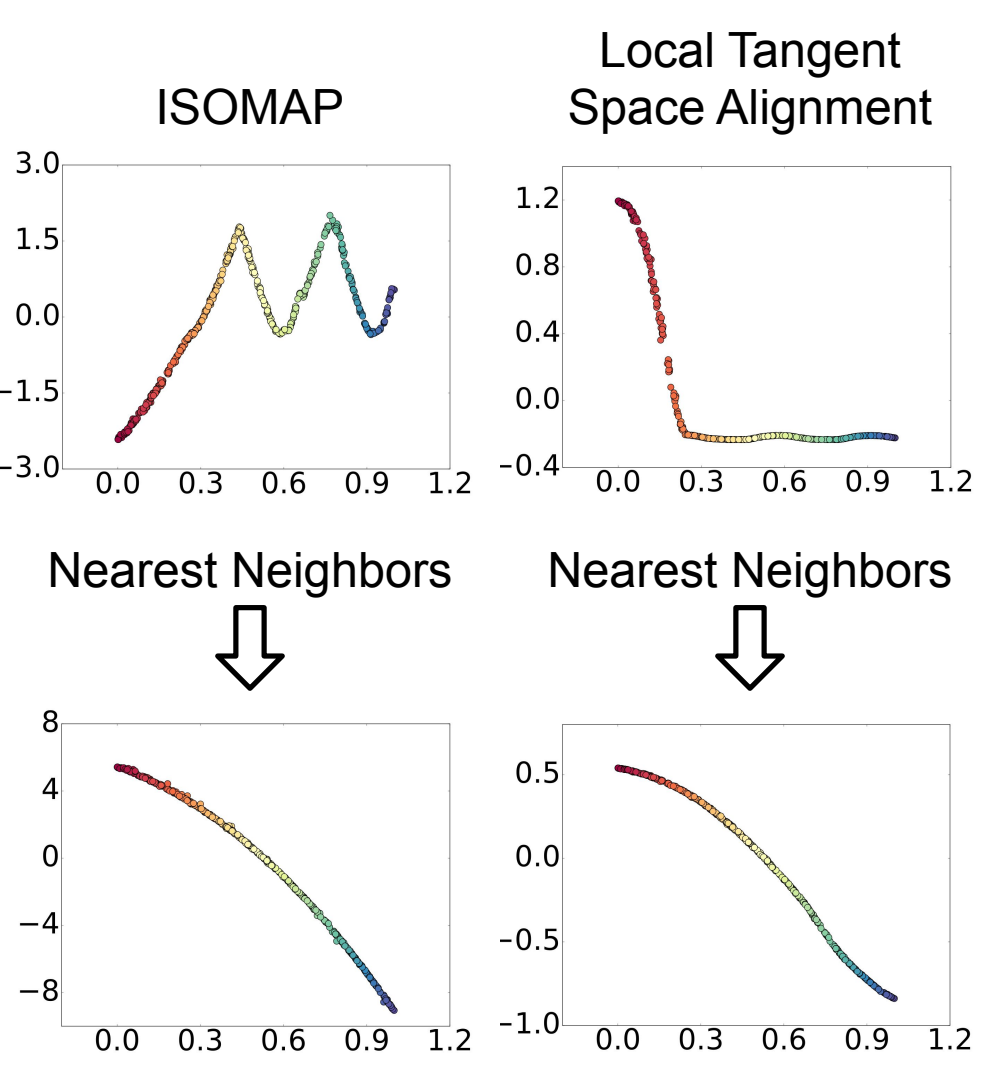


\section{Embedding Evaluation}
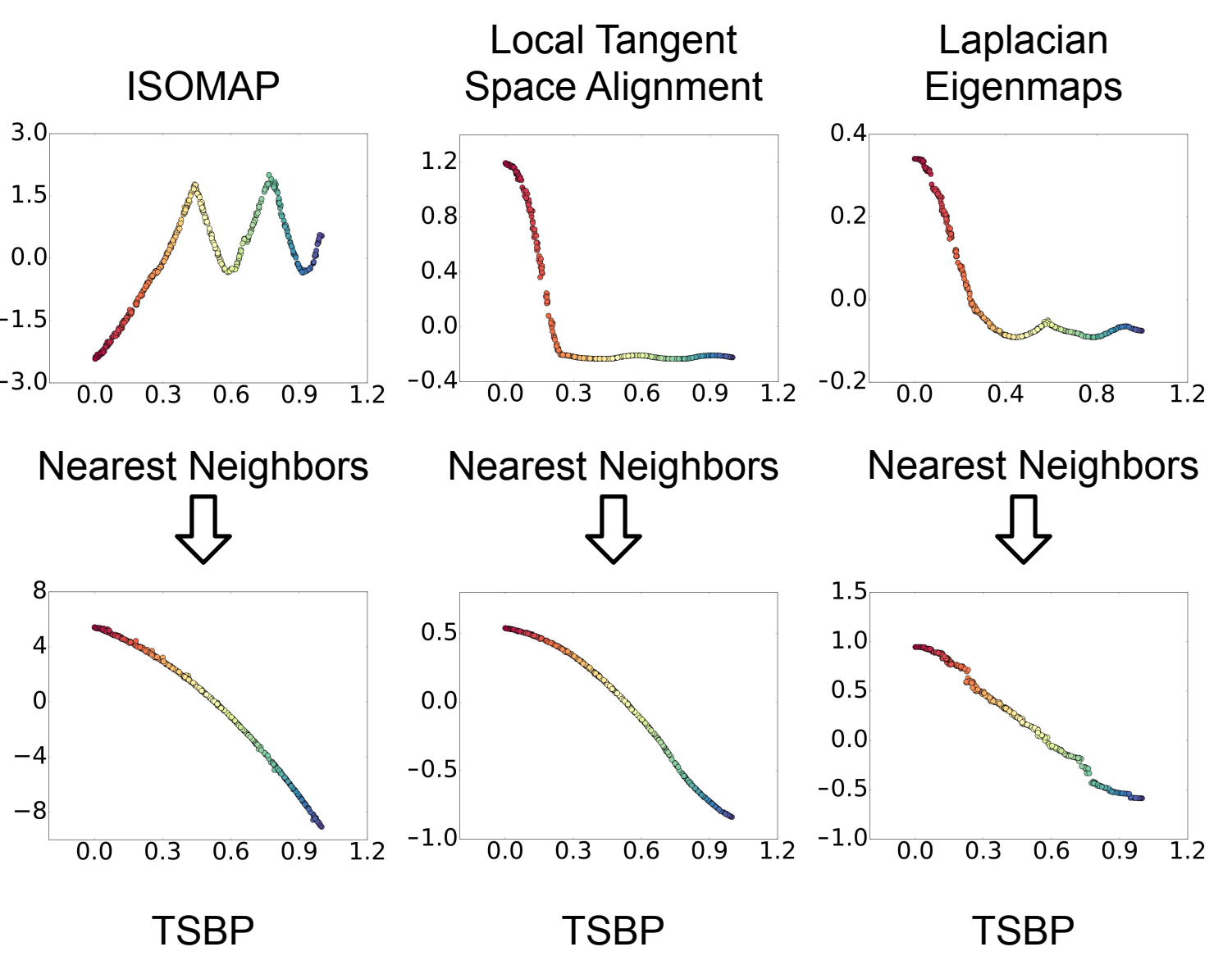


\section{Embedding Evaluation}
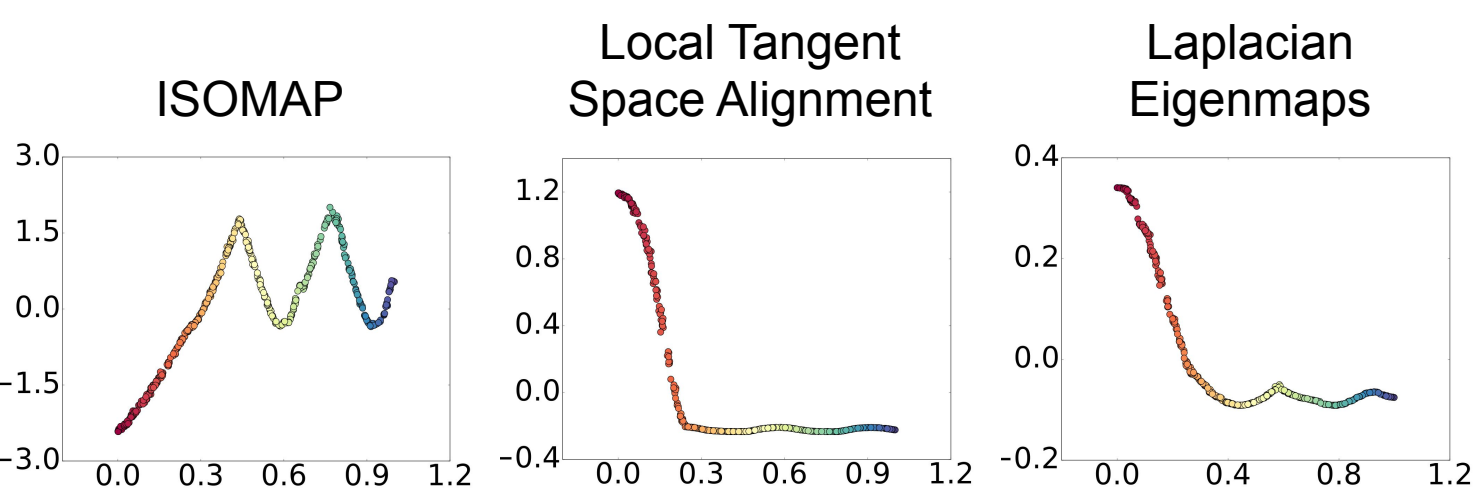

t-Distributed Stochastic Neighbor Embedding

Nearest Neighbors

Nearest Neighbors

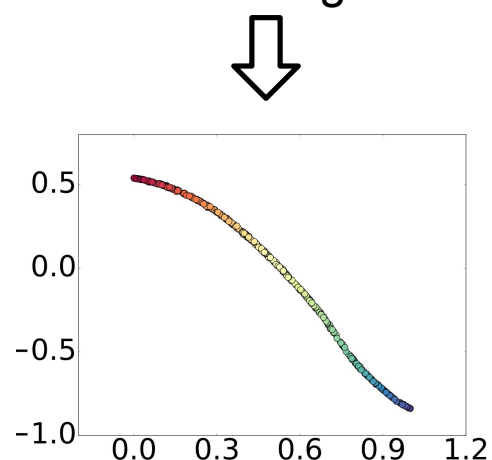

Nearest Neighbors
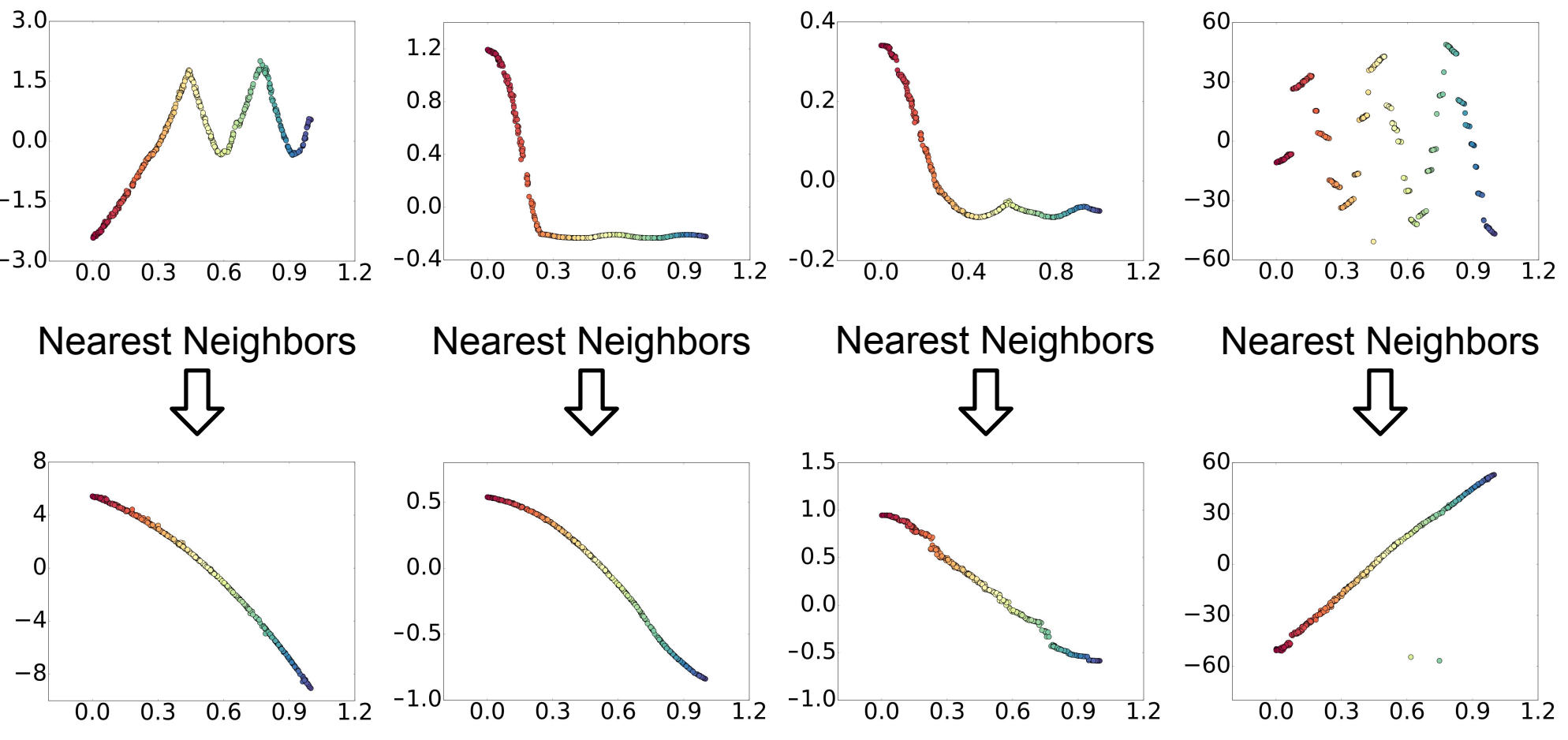

Nearest Neighbors 


\section{Embedding Evaluation}
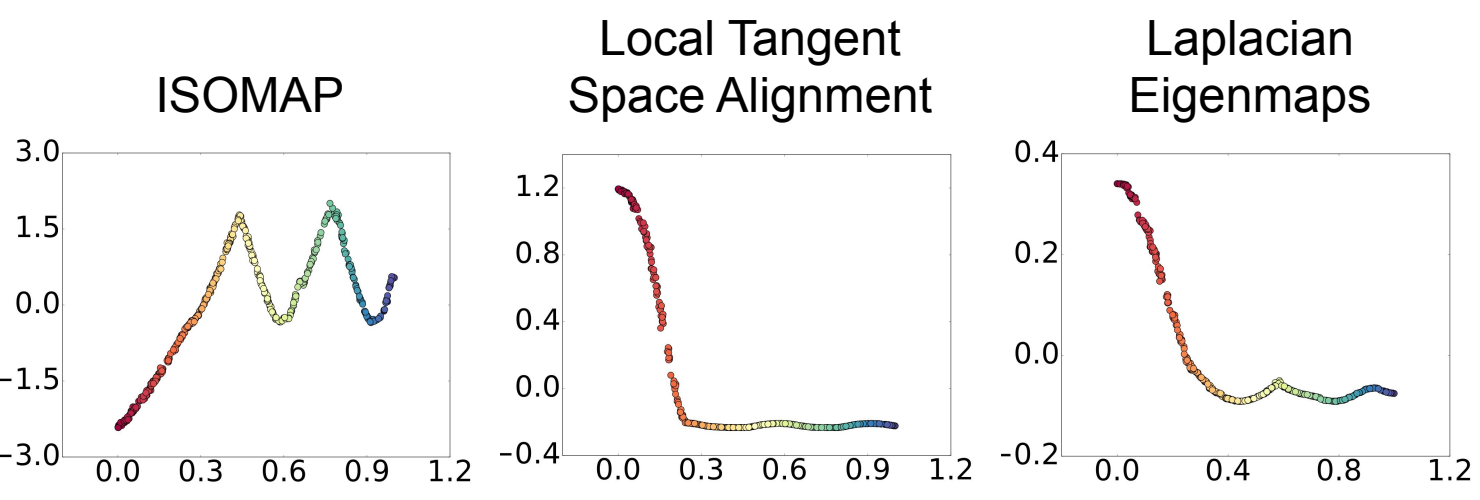

t-Distributed Stochastic Neighbor Embedding
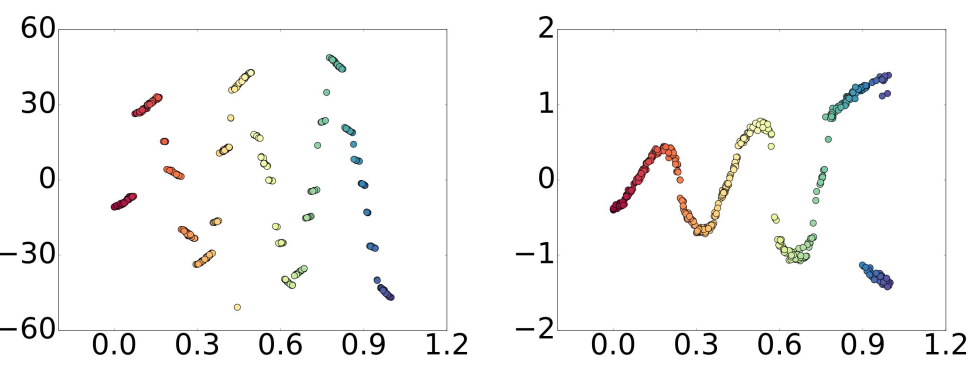

Nearest Neighbors

Nearest Neighbors

Nearest Neighbors

Nearest Neighbors

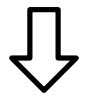

$\sqrt{ }$

Multidimensional

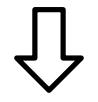

Scaling
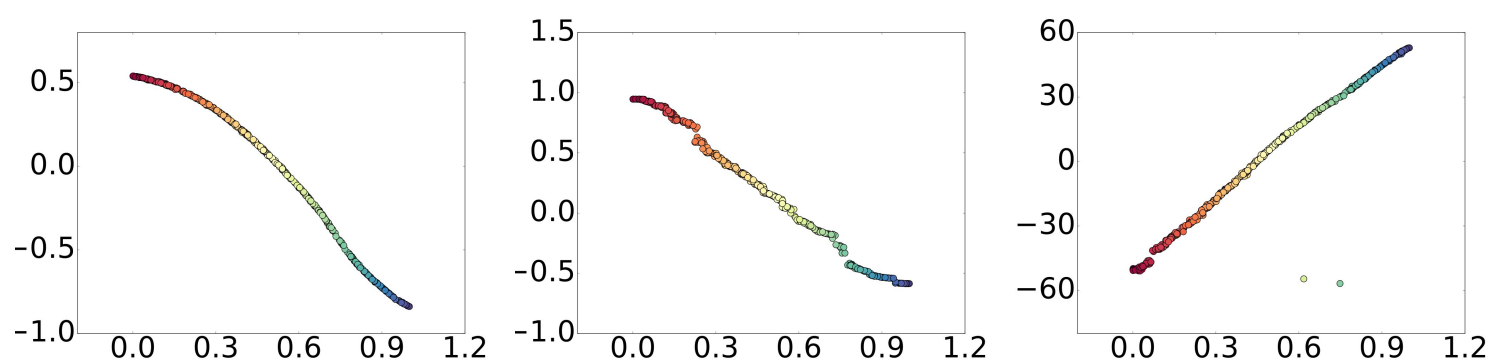


\section{Embedding Evaluation}

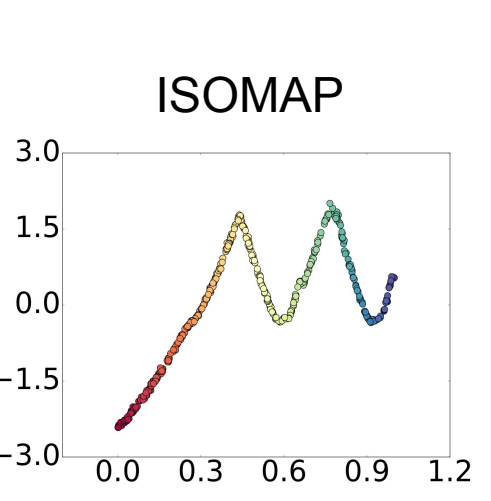

Local Tangent Space Alignment

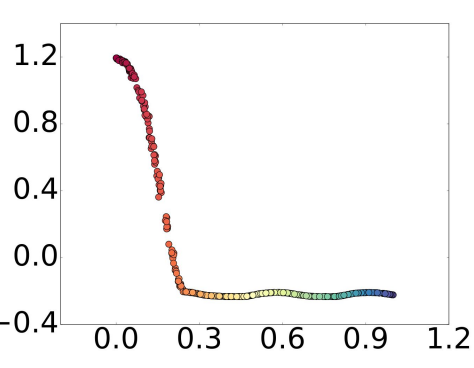

Nearest Neighbors
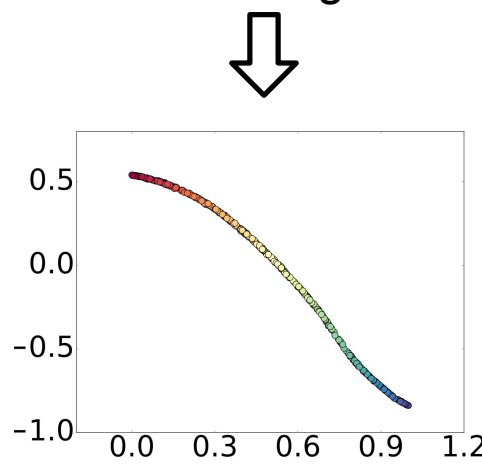

TSBP
Laplacian

Eigenmaps

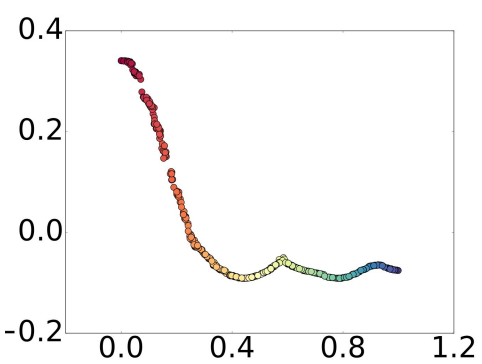

Nearest Neighbors
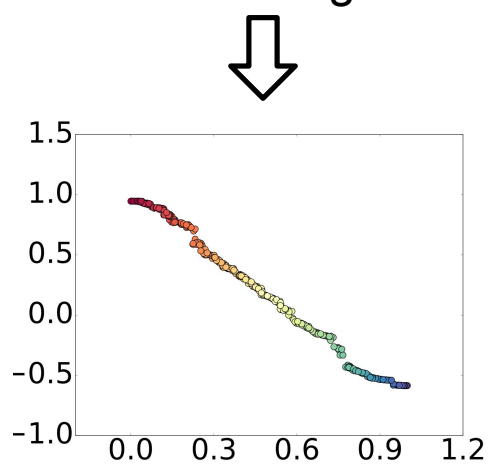

TSBP
t-Distributed Stochastic Neighbor Embedding

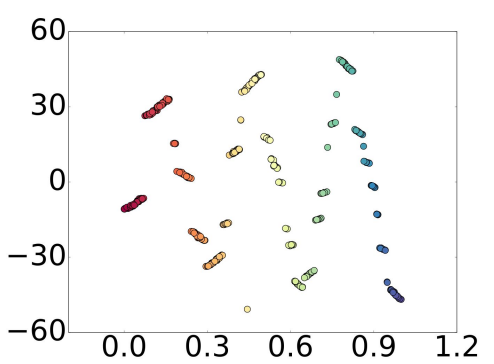

Nearest Neighbors
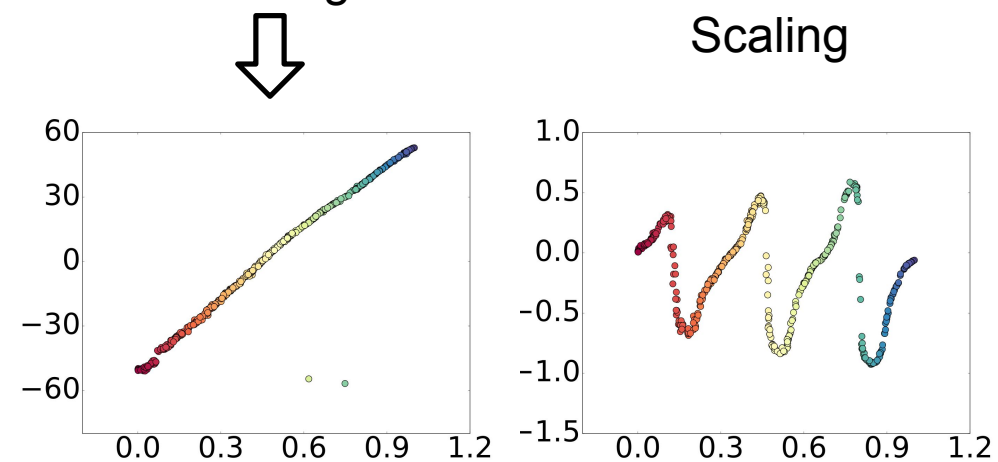

TSBP

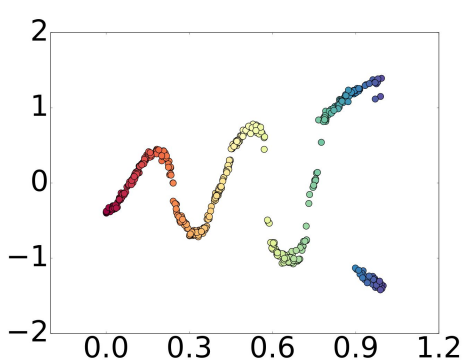

Multidimensional Scaling

Autoencoder ${ }^{62}$ 


\section{"Swiss Roll" Experiment}

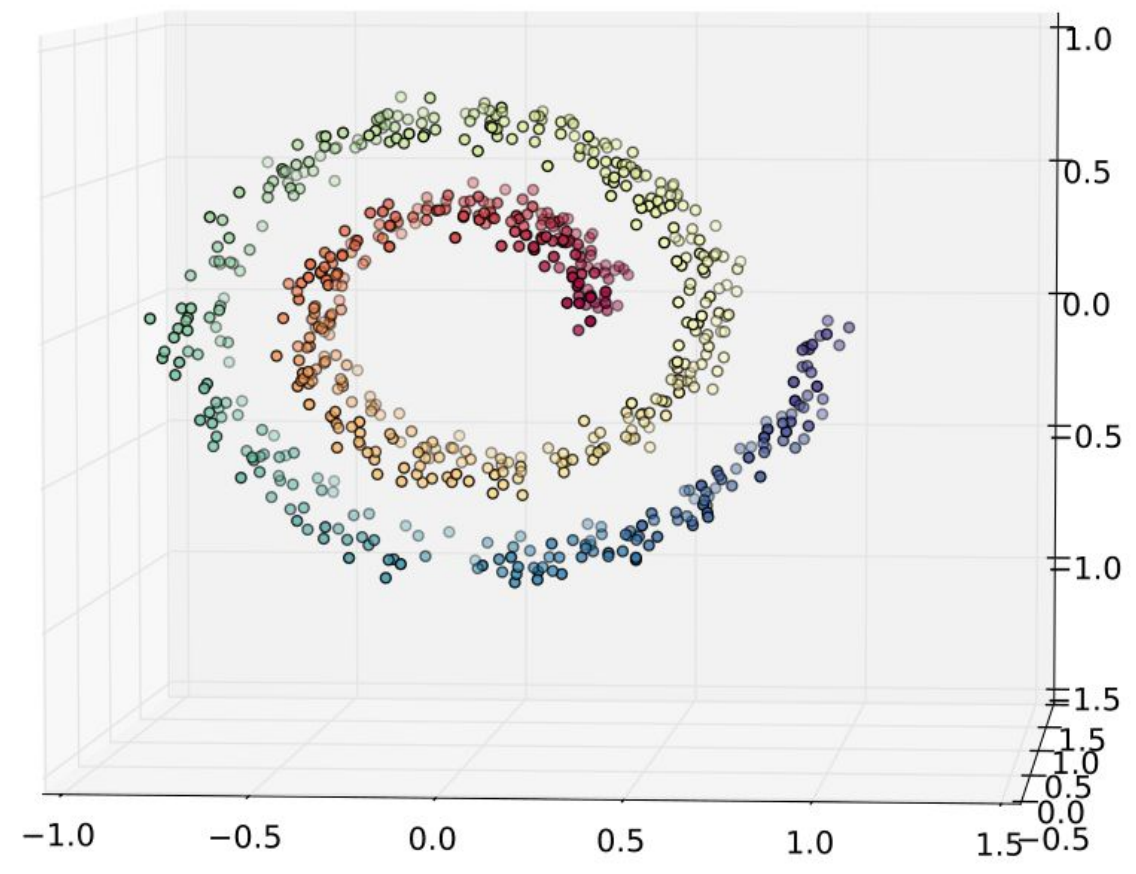




\section{"Swiss Roll" Experiment Neighbors}

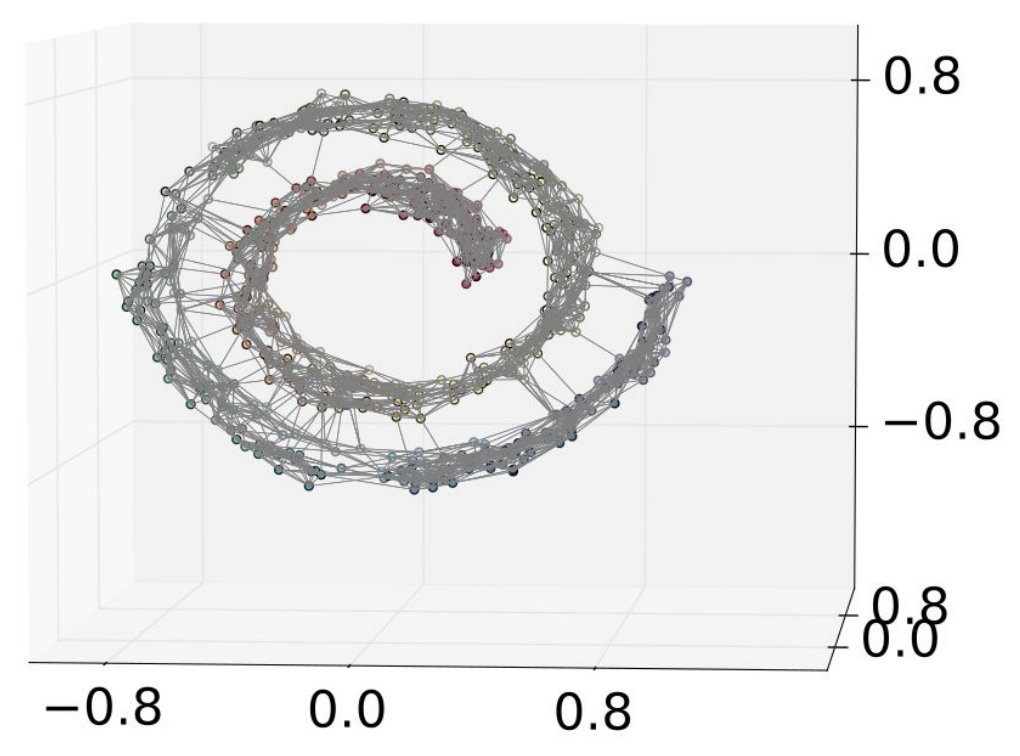

k-Nearest Neighbors

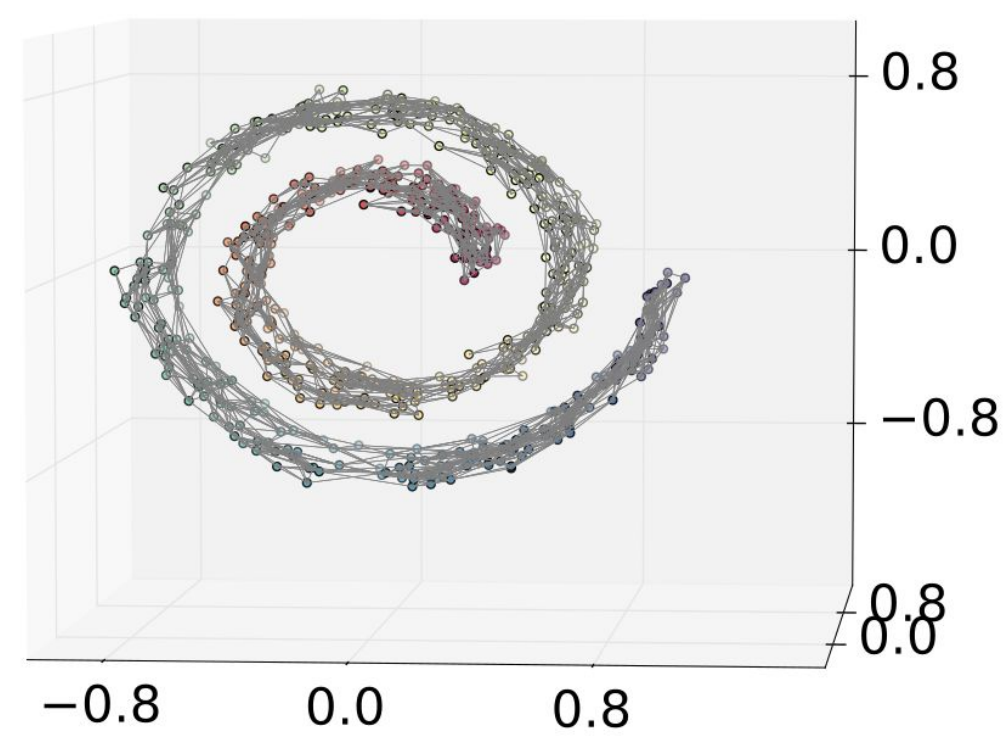

TSBP 


\section{Embedding Evaluation}

- Directly plot the $2 \mathrm{D}$ embedding

- Compare nearest-neighbors and TSBP

- Expect a rectangular embedding, with no data overlaps

- Ideal embedding:

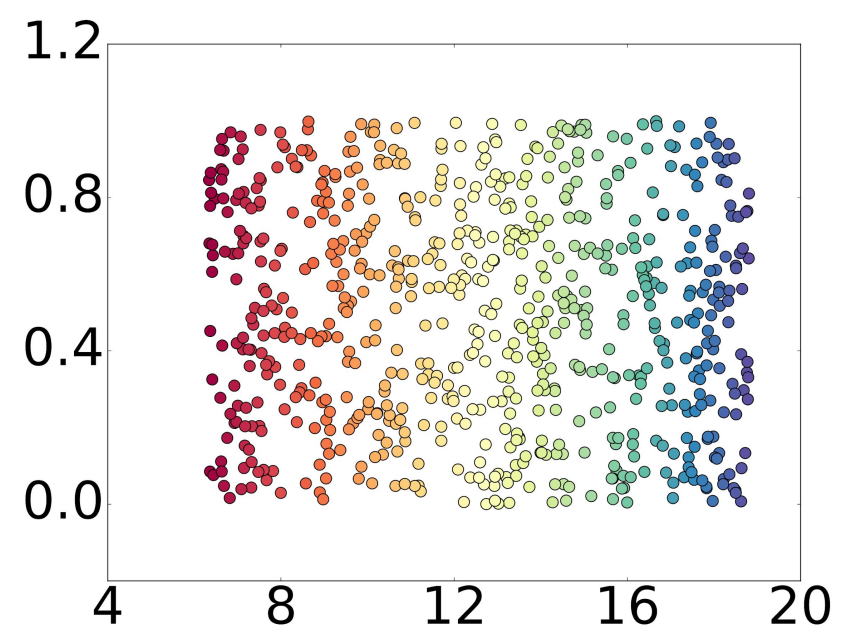




\section{Embedding Evaluation}

ISOMAP

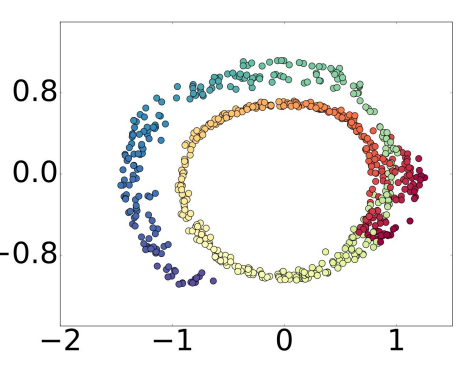

Nearest Neighbors

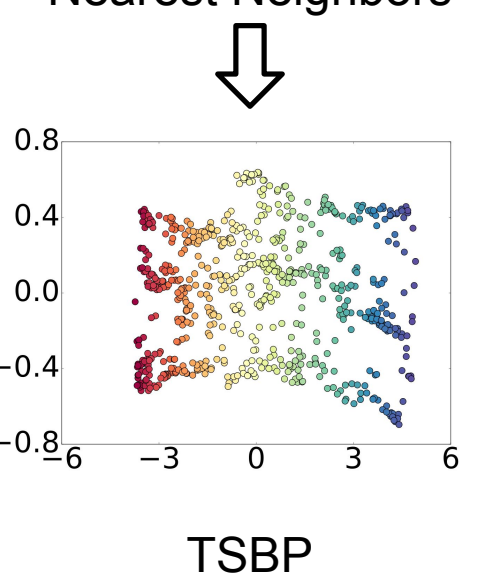

TSBP 


\section{Embedding Evaluation}

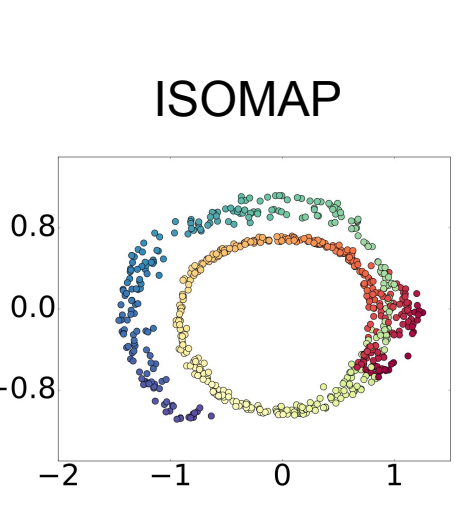

Local Tangent Space Alignment

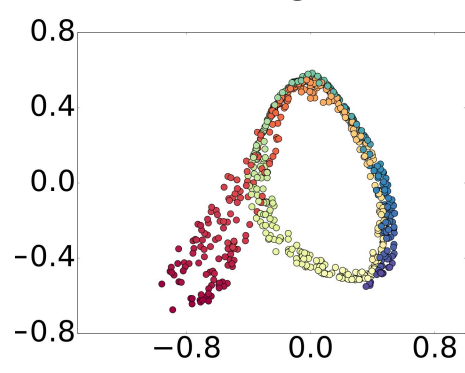

Nearest Neighbors

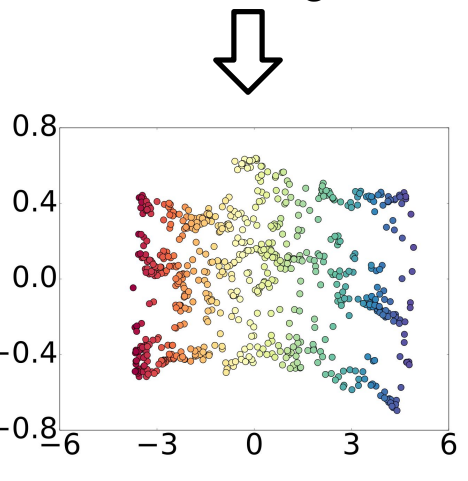

TSBP

Nearest Neighbors

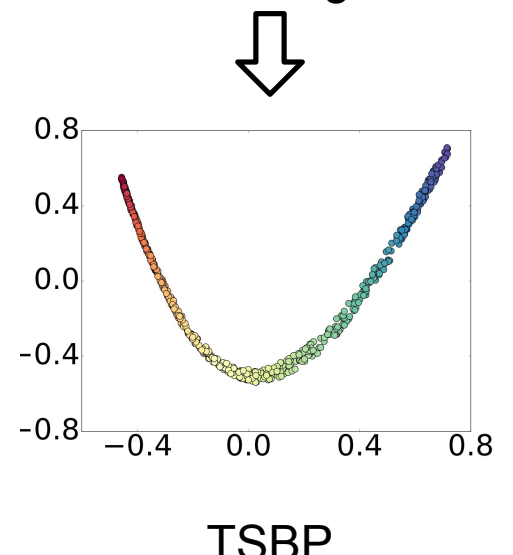




\section{Embedding Evaluation}
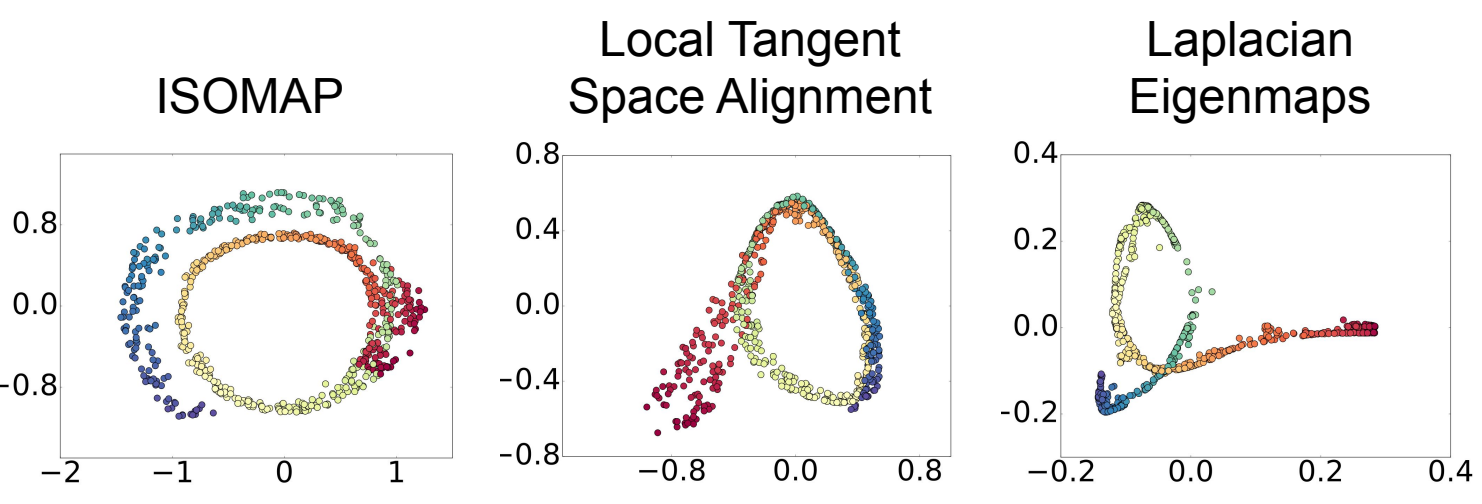

Nearest Neighbors

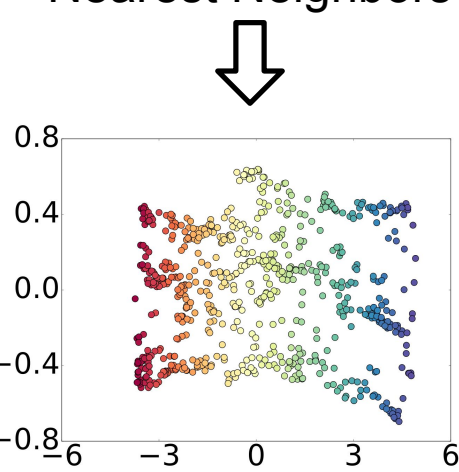

Nearest Neighbors
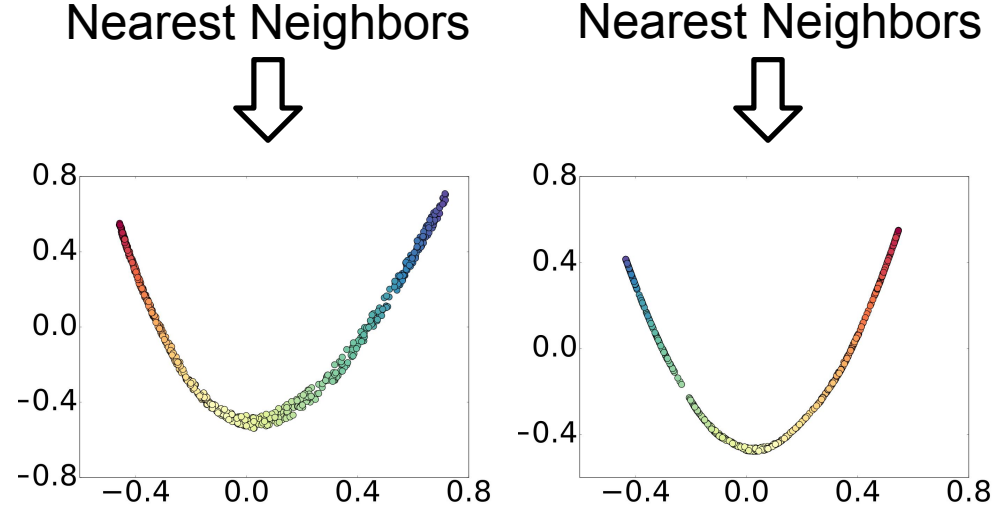

TSBP

TSBP

TSBP 


\section{Embedding Evaluation}
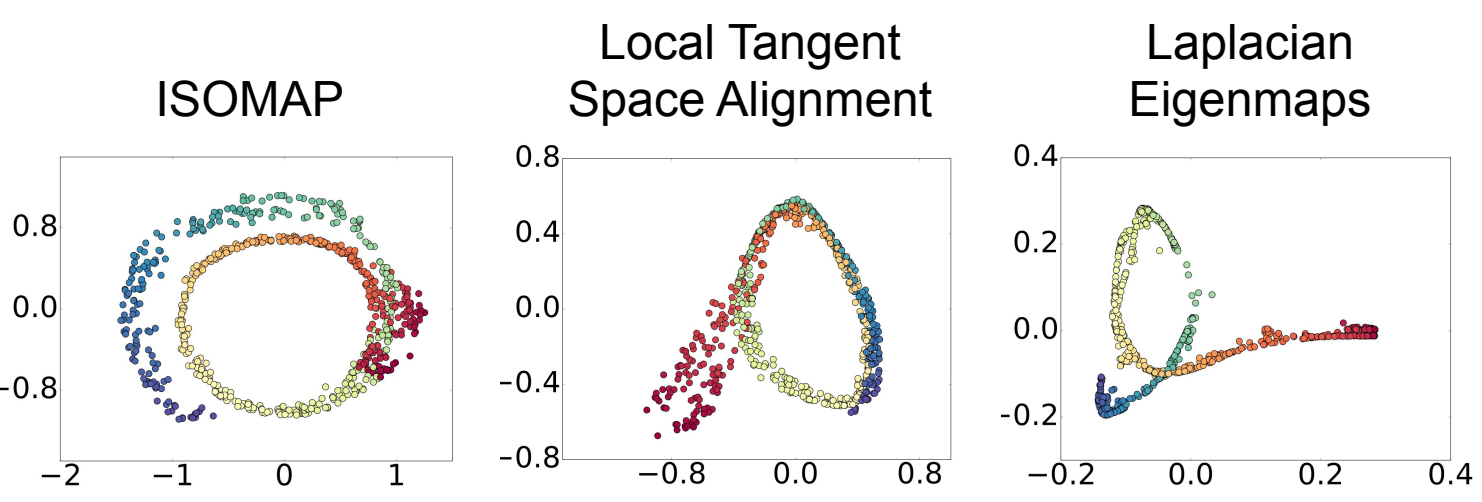

t-Distributed Stochastic Neighbor Embedding

Nearest Neighbors

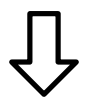

Nearest Neighbors
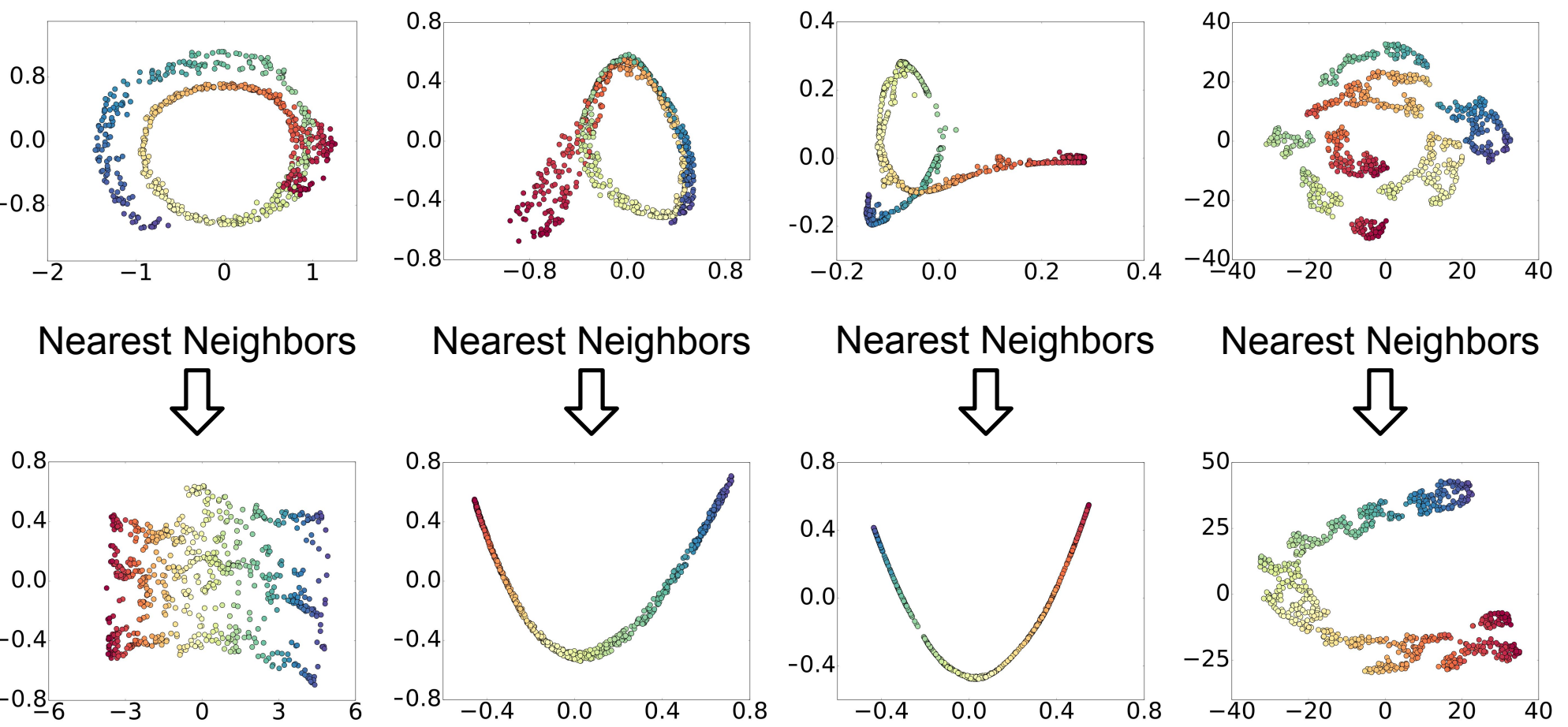

TSBP

TSBP

TSBP

TSBP 


\section{Embedding Evaluation}
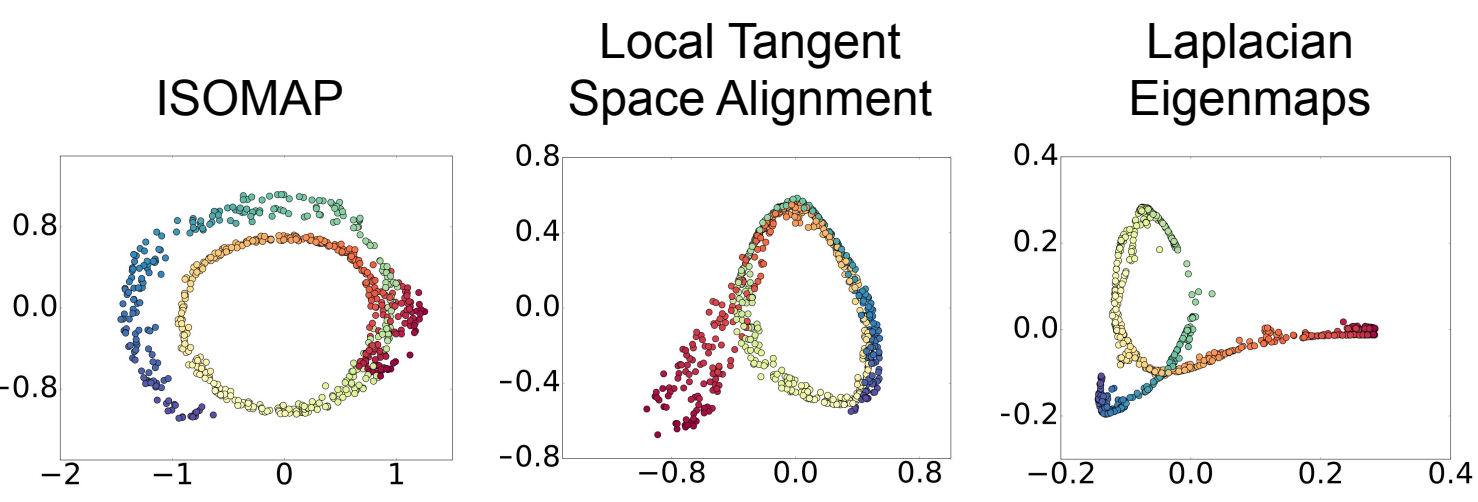

t-Distributed Stochastic Neighbor Embedding

Nearest Neighbors

Nearest Neighbors
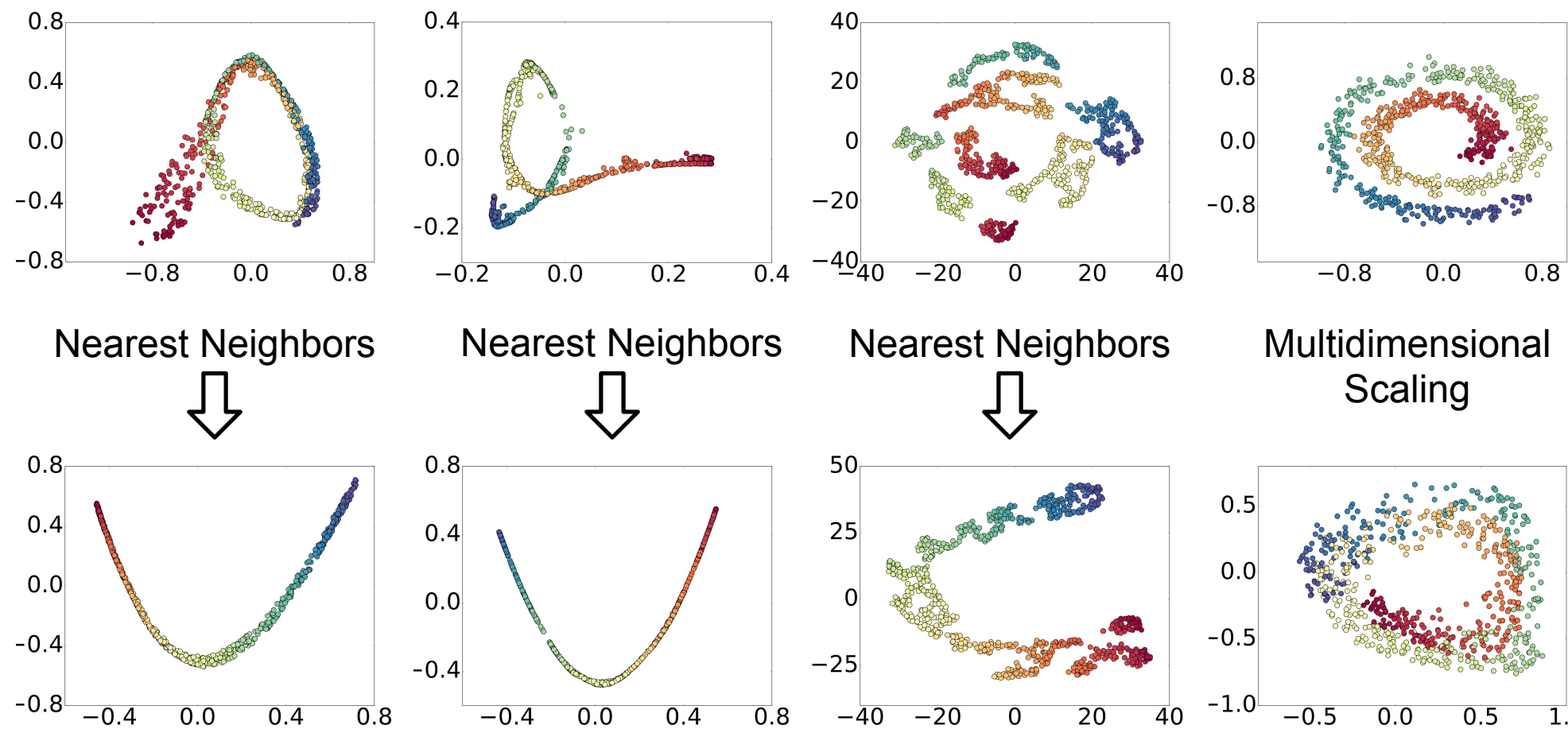

Multidimensional Scaling

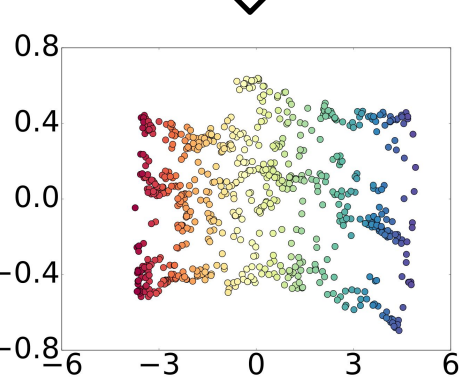

TSBP

TSBP

TSBP

TSBP

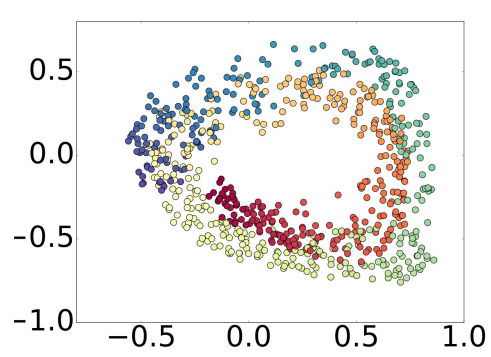

Autoencoder ${ }^{71}$ 


\section{High-Dimensional Tactile Data}

- Tactile sensing can be useful for intricate grasping and manipulation

- Scalable Tactile Glove (STAG) gathers detailed information from human grasp

- 548 force sensors (high dimensional data)

- Perform object classification with a Deep Convolutional Neural Network (Deep CNN)

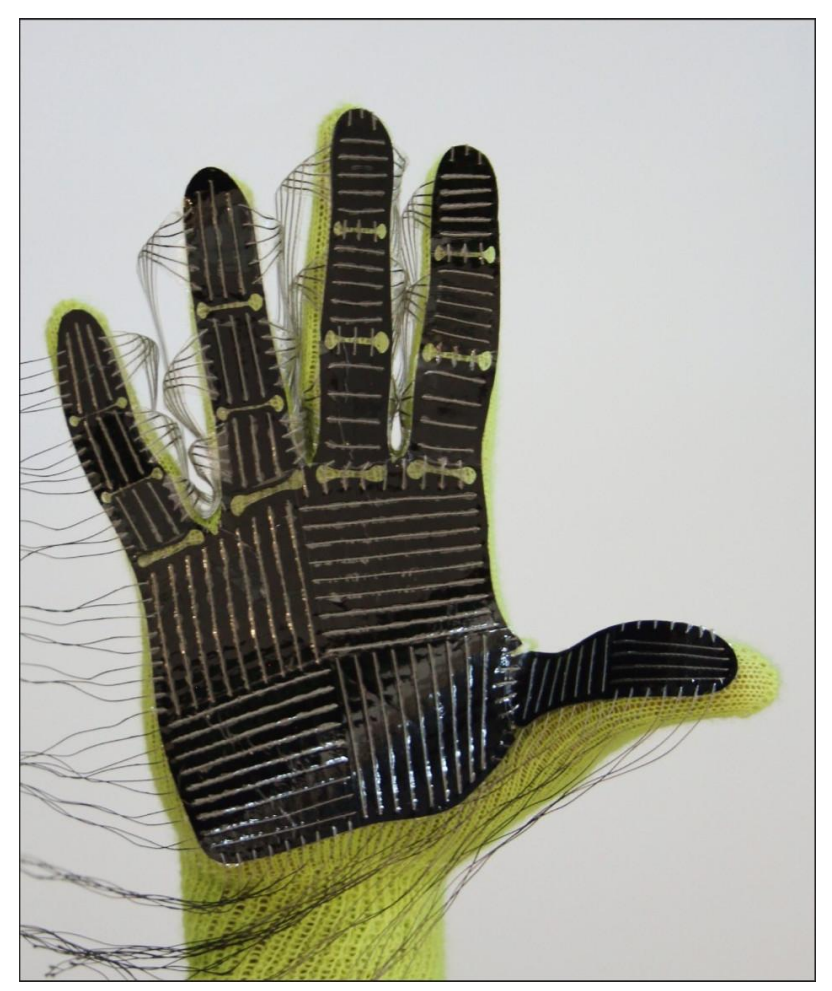

IMAGE: Learning the signatures of the human 


\section{Dimensionality Reduction and Classification}

- Want to perform object classification without large amounts of training data

- 26 objects (plus empty hand)

- 24 samples per class

- 50/50 train-test split

- Use a Gaussian Process Classifier (GPC) with RBF Kernel

- Kernel relies on Euclidean metric, which performs poorly in high dimensional spaces

- Perform dimensionality reduction with t-SNE 


\section{Tactile Data Classification Results}

\begin{tabular}{|c|c|c|c|c|c|c|}
\hline & $\begin{array}{c}\text { Random } \\
\text { Chance }\end{array}$ & $\begin{array}{c}\text { GPC with } \\
\text { Original Data }\end{array}$ & $\begin{array}{c}\text { GPC with } \\
\text { Ordinary t-SNE }\end{array}$ & $\begin{array}{c}\text { GPC with } \\
\text { Nearest-Neighbors } \\
\text { t-SNE }\end{array}$ & $\begin{array}{c}\text { GPC with } \\
\text { Autoencoder } \\
\text { Embedding }\end{array}$ & $\begin{array}{c}\text { Deep CNN } \\
\text { TSBP t-SNE }\end{array}$ \\
\hline Top-1 Accuracy & 0.0370 & & & & \\
\hline Top-3 Accuracy & 0.1111 & & & \\
\hline
\end{tabular}




\section{Tactile Data Classification Results}

\begin{tabular}{|l|c|c|c|c|c|c|c|}
\hline & $\begin{array}{c}\text { Random } \\
\text { Chance }\end{array}$ & $\begin{array}{c}\text { GPC with } \\
\text { Original Data }\end{array}$ & $\begin{array}{c}\text { GPC with } \\
\text { Ordinary t-SNE }\end{array}$ & $\begin{array}{c}\text { GPC with } \\
\text { Nearest-Neighbors } \\
\text { t-SNE }\end{array}$ & $\begin{array}{c}\text { GPC with } \\
\text { Autoencoder } \\
\text { Embedding }\end{array}$ & Deep CNN & $\begin{array}{c}\text { GPC with } \\
\text { TSBP t-SNE }\end{array}$ \\
\hline Top-1 Accuracy & 0.0370 & 0.0402 & & & & \\
\hline Top-3 Accuracy & 0.1111 & 0.1140 & & & & \\
\hline
\end{tabular}




\section{Tactile Data Classification Results}

\begin{tabular}{|l|c|c|c|c|c|c|c|}
\hline & $\begin{array}{c}\text { Random } \\
\text { Chance }\end{array}$ & $\begin{array}{c}\text { GPC with } \\
\text { Original Data }\end{array}$ & $\begin{array}{c}\text { GPC with } \\
\text { Ordinary t-SNE }\end{array}$ & $\begin{array}{c}\text { GPC with } \\
\text { Nearest-Neighbors } \\
\text { t-SNE }\end{array}$ & $\begin{array}{c}\text { GPC with } \\
\text { Autoencoder } \\
\text { Embedding }\end{array}$ & Deep CNN & $\begin{array}{c}\text { GPC with } \\
\text { TSBP t-SNE }\end{array}$ \\
\hline Top-1 Accuracy & 0.0370 & 0.0402 & 0.0421 & & & \\
\hline Top-3 Accuracy & 0.1111 & 0.1140 & 0.1156 & & & \\
\hline
\end{tabular}




\section{Tactile Data Classification Results}

\begin{tabular}{|l|c|c|c|c|c|c|c|}
\hline & $\begin{array}{c}\text { Random } \\
\text { Chance }\end{array}$ & $\begin{array}{c}\text { GPC with } \\
\text { Original Data }\end{array}$ & $\begin{array}{c}\text { GPC with } \\
\text { Ordinary t-SNE }\end{array}$ & $\begin{array}{c}\text { GPC with } \\
\text { Nearest-Neighbors } \\
\text { t-SNE }\end{array}$ & $\begin{array}{c}\text { GPC with } \\
\text { Autoencoder } \\
\text { Embedding }\end{array}$ & Deep CNN & $\begin{array}{c}\text { GPC with } \\
\text { TSBP t-SNE }\end{array}$ \\
\hline Top-1 Accuracy & 0.0370 & 0.0402 & 0.0421 & 0.0380 & & \\
\hline Top-3 Accuracy & 0.1111 & 0.1140 & 0.1156 & 0.1120 & & \\
\hline
\end{tabular}




\section{Tactile Data Classification Results}

\begin{tabular}{|l|c|c|c|c|c|c|c|}
\hline & $\begin{array}{c}\text { Random } \\
\text { Chance }\end{array}$ & $\begin{array}{c}\text { GPC with } \\
\text { Original Data }\end{array}$ & $\begin{array}{c}\text { GPC with } \\
\text { Ordinary t-SNE }\end{array}$ & $\begin{array}{c}\text { GPC with } \\
\text { Nearest-Neighbors } \\
\text { t-SNE }\end{array}$ & $\begin{array}{c}\text { GPC with } \\
\text { Autoencoder } \\
\text { Embedding }\end{array}$ & Deep CNN & $\begin{array}{c}\text { GPC with } \\
\text { TSBP t-SNE }\end{array}$ \\
\hline Top-1 Accuracy & 0.0370 & 0.0402 & 0.0421 & 0.0380 & 0.0612 & \\
\hline Top-3 Accuracy & 0.1111 & 0.1140 & 0.1156 & 0.1120 & 0.1551 & \\
\hline
\end{tabular}




\section{Tactile Data Classification Results}

\begin{tabular}{|l|c|c|c|c|c|c|c|}
\hline & $\begin{array}{c}\text { Random } \\
\text { Chance }\end{array}$ & $\begin{array}{c}\text { GPC with } \\
\text { Original Data }\end{array}$ & $\begin{array}{c}\text { GPC with } \\
\text { Ordinary t-SNE }\end{array}$ & $\begin{array}{c}\text { GPC with } \\
\text { Nearest-Neighbors } \\
\text { t-SNE }\end{array}$ & $\begin{array}{c}\text { GPC with } \\
\text { Autoencoder } \\
\text { Embedding }\end{array}$ & Deep CNN & $\begin{array}{c}\text { GPC with } \\
\text { TSBP t-SNE }\end{array}$ \\
\hline Top-1 Accuracy & 0.0370 & 0.0402 & 0.0421 & 0.0380 & 0.0612 & 0.0897 & \\
\hline Top-3 Accuracy & 0.1111 & 0.1140 & 0.1156 & 0.1120 & 0.1551 & 0.1888 & \\
\hline
\end{tabular}




\section{Tactile Data Classification Results}

\begin{tabular}{|l|c|c|c|c|c|c|c|}
\hline & $\begin{array}{c}\text { Random } \\
\text { Chance }\end{array}$ & $\begin{array}{c}\text { GPC with } \\
\text { Original Data }\end{array}$ & $\begin{array}{c}\text { GPC with } \\
\text { Ordinary t-SNE }\end{array}$ & $\begin{array}{c}\text { GPC with } \\
\text { Nearest-Neighbors } \\
\text { t-SNE }\end{array}$ & $\begin{array}{c}\text { GPC with } \\
\text { Autoencoder } \\
\text { Embedding }\end{array}$ & Deep CNN & $\begin{array}{c}\text { GPC with } \\
\text { TSBP t-SNE }\end{array}$ \\
\hline Top-1 Accuracy & 0.0370 & 0.0402 & 0.0421 & 0.0380 & 0.0612 & 0.0897 & $\mathbf{0 . 1 8 7 4}$ \\
\hline Top-3 Accuracy & 0.1111 & 0.1140 & 0.1156 & 0.1120 & 0.1551 & 0.1888 & $\mathbf{0 . 2 9 7 2}$ \\
\hline
\end{tabular}




\section{Tactile Data Classification Results}

\begin{tabular}{|l|c|c|c|c|c|c|}
\hline & $\begin{array}{c}\text { Random } \\
\text { Chance }\end{array}$ & $\begin{array}{c}\text { GPC with } \\
\text { Original Data }\end{array}$ & $\begin{array}{c}\text { GPC with } \\
\text { Ordinary t-SNE }\end{array}$ & $\begin{array}{c}\text { GPC with } \\
\text { Nearest-Neighbors } \\
\text { t-SNE }\end{array}$ & $\begin{array}{c}\text { GPC with } \\
\text { Autoencoder } \\
\text { Embedding }\end{array}$ & $\begin{array}{c}\text { GPC with } \\
\text { TSBP t-SNE }\end{array}$ \\
\hline Top-1 Accuracy & 0.0370 & 0.0402 & 0.0421 & 0.0380 & 0.0612 & 0.1874 \\
\hline Top-3 Accuracy & 0.1111 & 0.1140 & 0.1156 & 0.1120 & 0.1551 \\
\hline
\end{tabular}
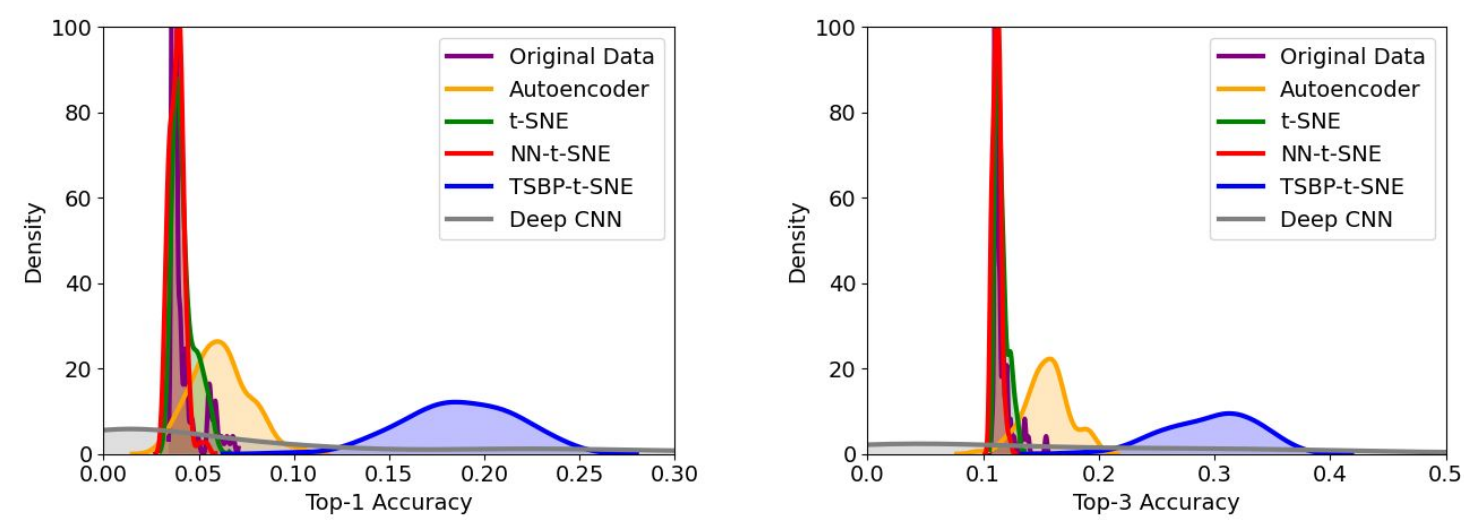


\section{Tactile Data Classification Results}

\begin{tabular}{|l|c|c|c|c|c|c|}
\hline & $\begin{array}{c}\text { Random } \\
\text { Chance }\end{array}$ & $\begin{array}{c}\text { GPC with } \\
\text { Original Data }\end{array}$ & $\begin{array}{c}\text { GPC with } \\
\text { Ordinary t-SNE }\end{array}$ & $\begin{array}{c}\text { GPC with } \\
\text { Nearest-Neighbors } \\
\text { t-SNE }\end{array}$ & $\begin{array}{c}\text { GPC with } \\
\text { Autoencoder } \\
\text { Embedding }\end{array}$ & $\begin{array}{c}\text { GPC with } \\
\text { TSBP t-SNE }\end{array}$ \\
\hline Top-1 Accuracy & 0.0370 & 0.0402 & 0.0421 & 0.0380 & 0.0612 & 0.1874 \\
\hline Top-3 Accuracy & 0.1111 & 0.1140 & 0.1156 & 0.1120 & 0.1551 \\
\hline
\end{tabular}
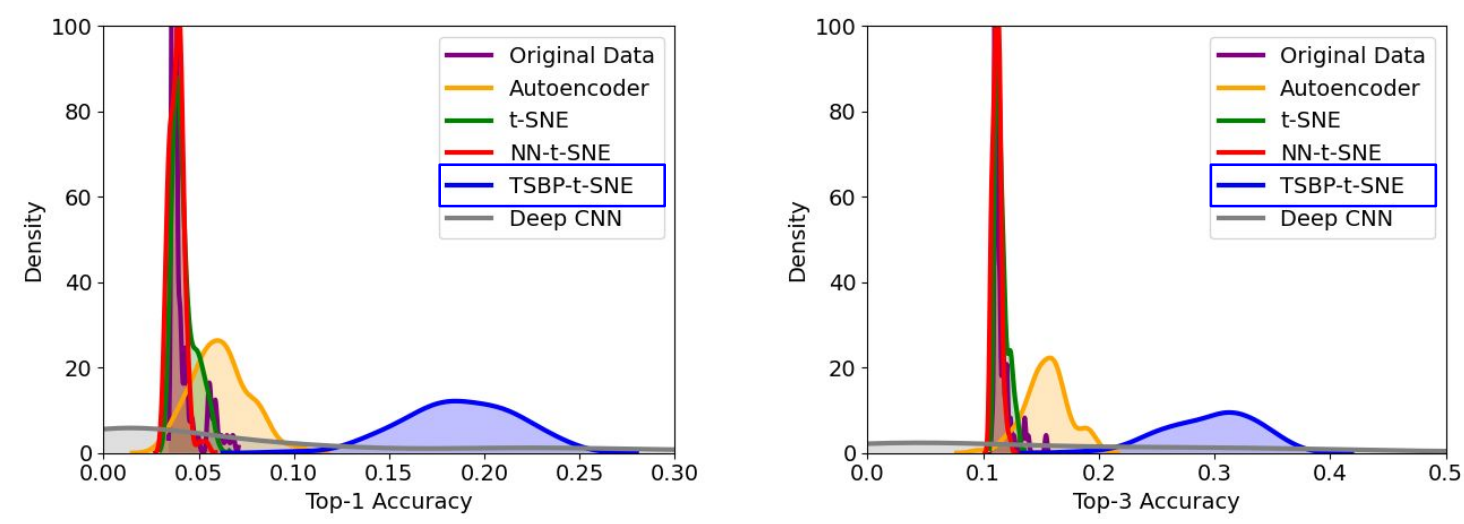


\section{Conclusion}

- High dimensional data is inherently difficult to work with

- Manifold learning is one solution to this "Curse of Dimensionality"

- Sparse and noisy data can cause manifold learning to fail 


\section{Conclusion}

- High dimensional data is inherently difficult to work with

- Manifold learning is one solution to this "Curse of Dimensionality"

- Sparse and noisy data can cause manifold learning to fail

- TSBP makes manifold learning more robust

- Accurate tangent space estimates are obtained with belief propagation

- False edges can be identified by comparing with manifold tangents

- These edges are removed to produce a denoised neighborhood graph

- This allows existing manifold learning algorithms to produce a more accurate embedding 


\section{TSBP: Tangent Space Belief Propagation for Manifold Learning}

Thomas Cohn, Odest Chadwicke Jenkins, Karthik Desingh, Zhen Zeng

\section{IROS 2020}

Laboratory for

Perception RObotics and Grounded REasoning SystemS 


\section{Potential Functions}

Given bases:

$$
U=\left(u_{1}, \ldots, u_{k}\right), V=\left(v_{1}, \ldots, v_{k}\right)
$$

Vector subspace dissimilarity:

$$
\Gamma(U, V)=\sum_{i=1}^{k}\left\|u_{i}-\operatorname{proj}_{V} u_{i}\right\|^{2}
$$

Unary Potential:

$$
\phi\left(\mathcal{T}_{i}, \mathcal{Y}_{i}\right)=\left(1+\Gamma\left(\mathcal{T}_{i}, \mathcal{Y}_{i}\right)\right)^{-1}
$$

Pairwise Potential:

$$
\psi\left(\mathcal{T}_{i}, \mathcal{T}_{j}\right)=\left(1+\Gamma\left(\mathcal{T}_{i}, \mathcal{T}_{j}\right)\right)^{-1}
$$




\section{Outliers}

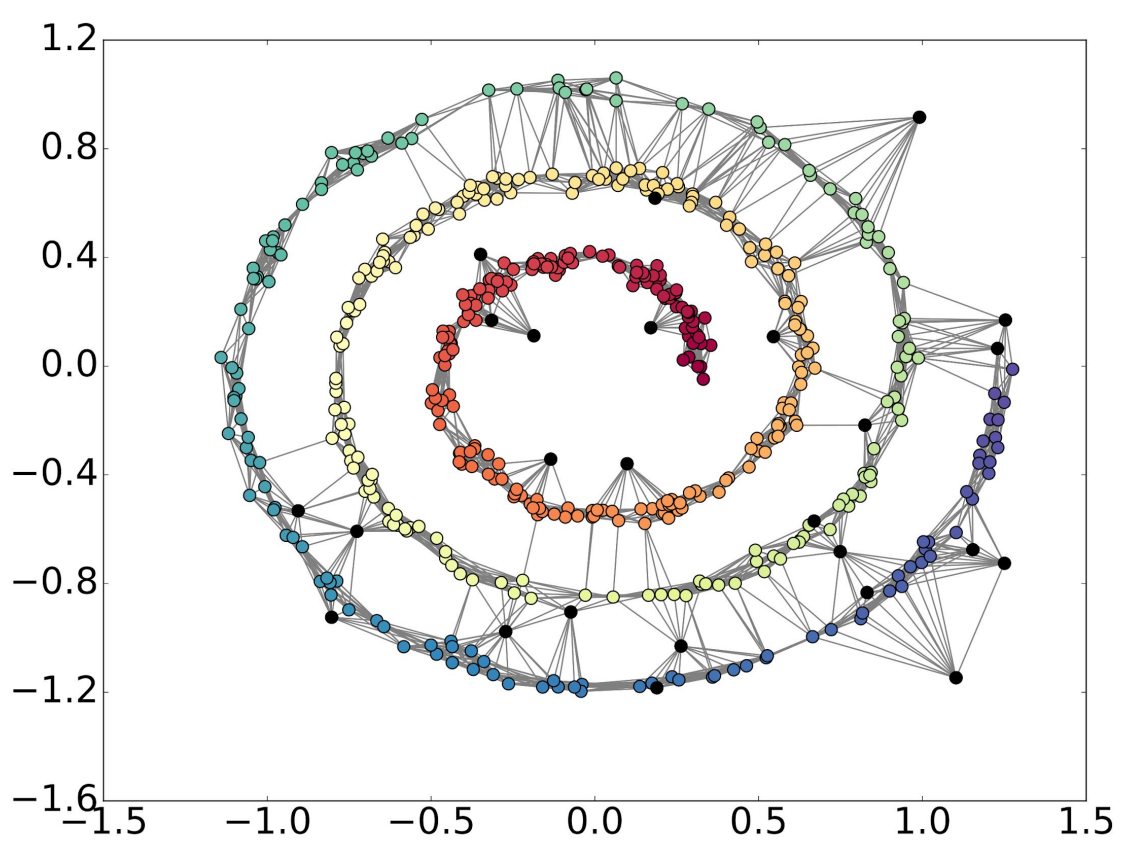

k-Nearest Neighbors

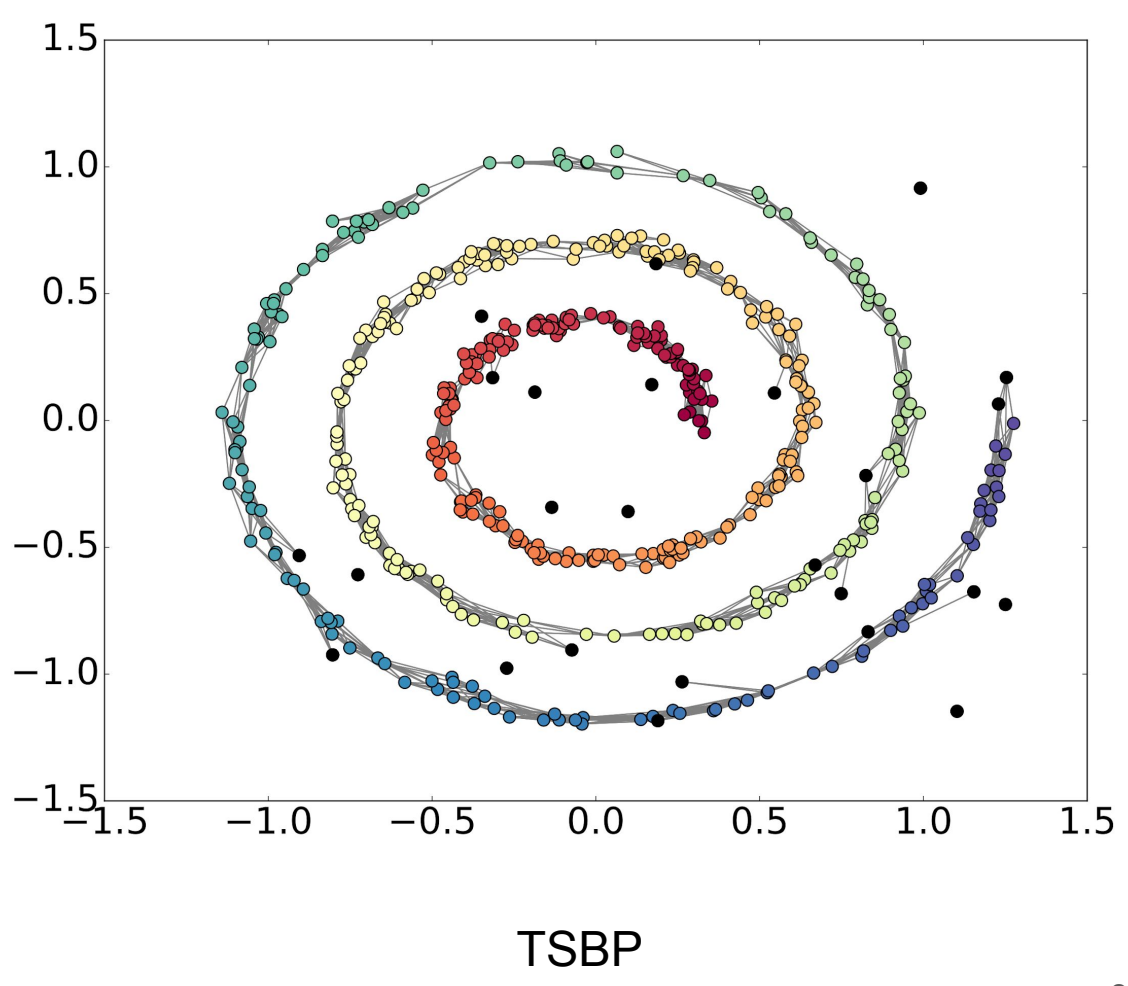




\section{L2-L1 PCA Comparison}
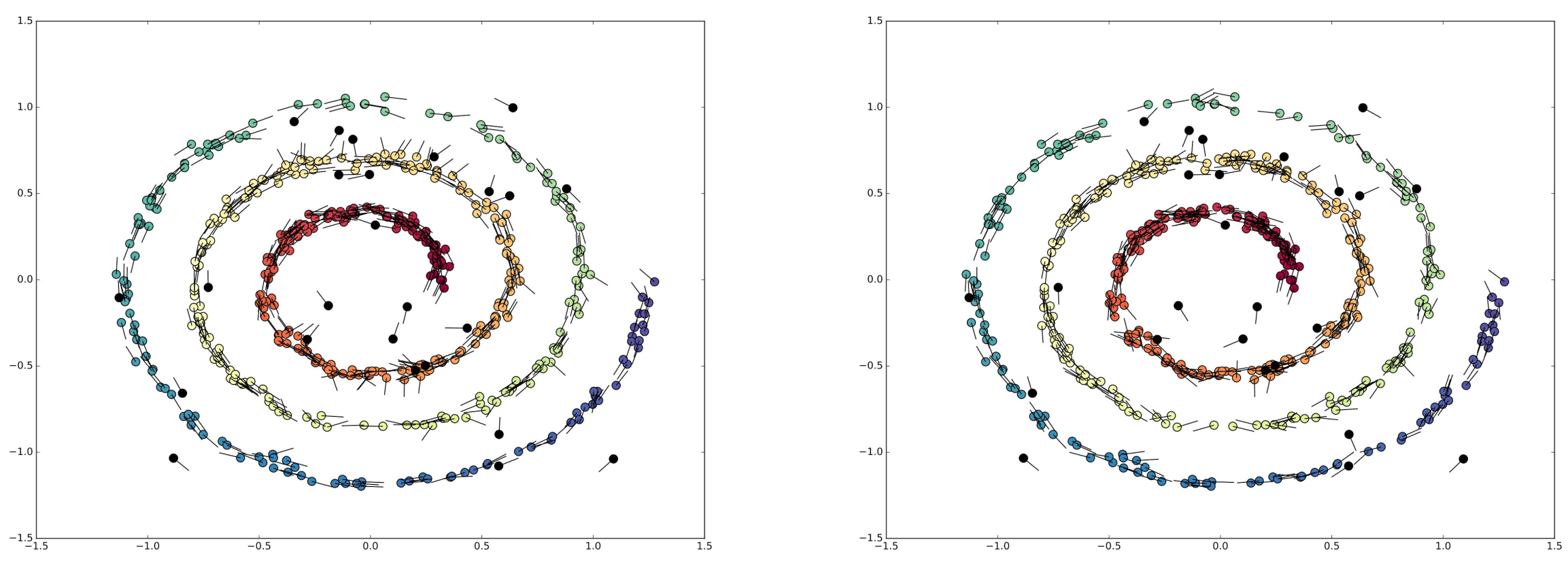

L2-PCA

L1-PCA 